University of Louisville ThinkIR: The University of Louisville's Institutional Repository

Electronic Theses and Dissertations

$5-2016$

\title{
ABIN1 in the pathogenesis of Glomerulonephritis and the novel polocyte-neutrophil proinflammatory axis.
}

Erik Anderson Korte

University of Louisville

Follow this and additional works at: https://ir.library.louisville.edu/etd

Part of the Biological Phenomena, Cell Phenomena, and Immunity Commons

\section{Recommended Citation}

Korte, Erik Anderson, "ABIN1 in the pathogenesis of Glomerulonephritis and the novel polocyte-neutrophil proinflammatory axis." (2016). Electronic Theses and Dissertations. Paper 2464.

https://doi.org/10.18297/etd/2464

This Doctoral Dissertation is brought to you for free and open access by ThinkIR: The University of Louisville's Institutional Repository. It has been accepted for inclusion in Electronic Theses and Dissertations by an authorized administrator of ThinkIR: The University of Louisville's Institutional Repository. This title appears here courtesy of the author, who has retained all other copyrights. For more information, please contact thinkir@louisville.edu. 


\title{
ABIN1 IN THE PATHOGENESIS OF GLOMERULONEPHRITIS AND THE NOVEL PODOCYTE-NEUTROPHIL PROINFLAMMATORY AXIS
}

By

Erik Anderson Korte

B.S., Missouri State University, 2003

M.S., University of Louisville, 2012

\begin{abstract}
A Dissertation
Submitted to the Faculty of the School of Medicine of the University of Louisville in Partial Fulfillment of the Requirements for the Degree of

Doctor of Philosophy

in

Biochemistry and Molecular Biology

Department of Biochemistry and Molecular Genetics

University of Louisville Louisville, KY
\end{abstract}

May, 2016 
Copyright 2016 by Erik Anderson Korte

\author{
All right reserved
}





\title{
ABIN1 IN THE PATHOGENESIS OF GLOMERULONEPHRITIS AND THE NOVEL PODOCYTE-NEUTROPHIL PROINFLAMMATORY AXIS
}

\author{
By
}

Erik Anderson Korte

B.S., Missouri State University, 2003

M.S., University of Louisville, 2012

A Dissertation Approved on

$$
\text { April 8 }{ }^{\text {th }}, 2016
$$

by the following Dissertation Committee:

Dr. David W. Powell, Dissertation Director

Dr. Barbara J. Clark, Committee Member

Dr. Michele M. Kosiewicz, Committee Member

Dr. Russell A. Prough, Committee Member

Dr. Kenneth S. Ramos, Committee Member 


\section{DEDICATION}

I dedicate this dissertation to my family. I can never repay you for your unconditional

love and support and the opportunities you have given me. In particular, I dedicate this work to the family members we have lost. I miss you every day, but in that way I will never forget the things you taught me.

My Beloved Grandparents

Marie Anderson

Forrest W. Anderson

Anthony Korte

When someone you love becomes a memory, that memory becomes a treasure. 


\section{ACKNOWLEDGEMENTS}

I would like to take this opportunity to thank my incredible wife, Sarah Korte, for her love and support over the last 10 years that we have known each other. Sarah, you have been the most stable element in my life and I can’t express how thankful I am to have met you. Thank you for giving me Mackenzie. She is incredible and I love the time we get to spend with her. To Kenzie, keep working on those words. You have more to say than “Duck” and “Dada”. To my parents, Karen and Gary Korte, who have sacrificed so much for me, thank you. I was not an easy child to deal with, but I have tried to make you proud. You taught me how to work hard and you never let me doubt myself. To my brother, Ian, thanks for being easy to talk to and quick with a joke. To my sister, Erin, and her husband, Jayson, name your son Erik. It's a strong name and will destine him for great things. If you go with something else, I'm sure things will still work out for him. To my grandparents, you mean so much to me. I miss you terribly and will try to live in the way you wanted. I wish you could have met Kenzie. You would have liked her.

To my friends, past and current, we’ve had fun. We may be separated by just a few blocks or entire oceans, but I'll be there for you like you have been there for me. And thanks for agreeing that our mistakes "seemed like the right thing to do at the time".

To my committee, Dr. Clark, Dr. Kosiewicz, Dr. Prough and Dr. Ramos, thank you for your guidance in developing my project. To my labmates, Tim, Ryan and Dawn, 
you have helped me in ways that I haven’t always articulated. Tim, you taught me most of my laboratory techniques, at least initially, before Google taught me the correct method. And I believe you when you say you never contaminated the cell culture stocks. Ryan, you have always kept me honest when I come in with a crazy idea that I know won't work. Dawn, thank you for your clinical insight and for helping me focus on what will have the greatest impact on patients. And finally, David Powell, thank you for being the kind of mentor that allows me the freedom to follow whatever avenue seems right at the time. But more importantly, thank you for bringing me back to focus when I truly needed it. You provided the perfect environment to combine learning with productivity, and I could not ask for a better mentor. 


\begin{abstract}
ABIN1 IN THE PATHOGENESIS OF GLOMERULONEPHRITIS AND THE NOVEL PODOCYTE-NEUTROPHIL PROINFLAMMATORY AXIS

Erik Anderson Korte

April 8 ${ }^{\text {th }}, 2016$
\end{abstract}

Glomerulonephritis (GN) is the most common cause of end-stage renal disease worldwide and is characterized by deposition of immune complex, inflammation, proteinuria and podocyte damage. Immunosuppressive therapy is effective in less than $50 \%$ of patients, indicating that immunological activity is not the only relevant cause of kidney injury. While methods have been described for neutrophil-mediated immune activation, specific glomerular mechanisms for recruitment of neutrophils and development of GN have not been determined.

Here I present a pathogenic mechanism for the development of GN involving a novel podocyte-neutrophil axis and reveal the importance of glomerular NF-kB inhibition by the ubiquitin binding protein, ABIN1. I implicate enhanced glomerular NF-kB activity in human chronic kidney disease using transcriptomic analysis and link genetic ABIN1 polymorphisms to increased risk of lupus-related GN. Mice expressing dysfunctional ABIN1 with disrupted polyubiquitin binding activity are shown to develop spontaneous, progressive glomerular disease. Prevention and early diagnosis require an 
understanding of the initiating steps of disease pathogenesis, so early pathogenic changes were examined using antibodies directed against the mouse glomerular basement membrane to induce rapid glomerular damage. Glomeruli of mice expressing dysfunctional ABIN1 exhibit increased production of NF- $\mathrm{\kappa B}$ target cytokines and rapid induction of GN versus wild type mice. Bone marrow transplantation reveals the glomerular genotype is more critical than the immune genotype following insult, further supporting the role of the target tissue in early disease pathogenesis. I outline the contributions of mass spectrometry to the discovery of modulating factors and disease biomarkers in autoimmunity and describe an optimized technique to characterize the secreted protein milieu of cultured human podocytes. Dysfunctional ABIN1 results in enhanced cytokine production by podocytes that can recruit and activate primary neutrophils. Further, the podocytes are more sensitive to pathogenic cytoskeletal remodeling following exposure to neutrophil granules, highlighting a reciprocal injurious mechanism. Finally, a novel peptide inhibitor of neutrophil granule release attenuates antibody-induced proteinuria in these mice and may have therapeutic potential in human GN. Thus, I describe a novel podocyte-neutrophil axis in the pathogenesis of GN and propose a potential molecular mechanism of GN involving loss of ABIN1 function resulting in excessive NF- $\mathrm{KB}$ activation. 
TABLE OF CONTENTS

PAGE

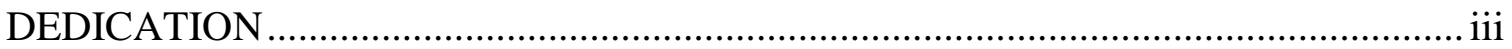

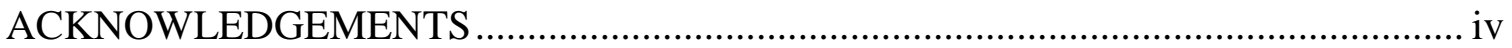

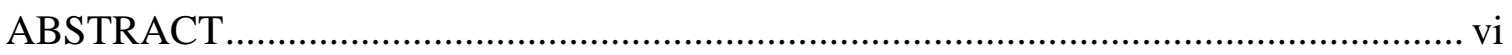

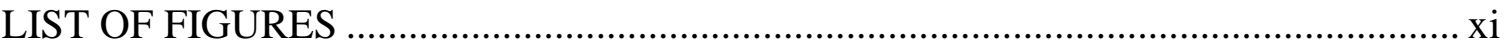

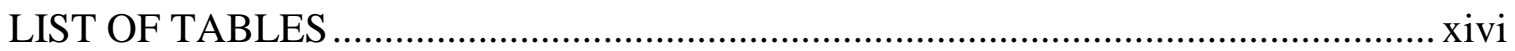

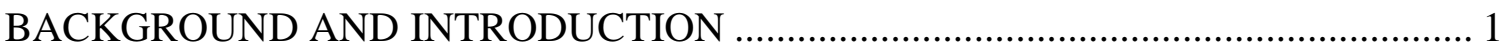

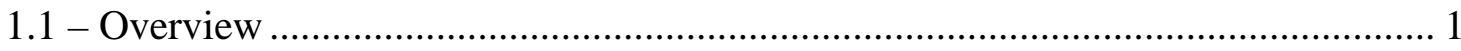

1.2 - Renal Anatomy and the Cellular Pathogenesis of Glomerular Inflammation........ 4

1.3 - Nuclear Factor kappa B in Inflammation and Disease................................... 11

1.4 - ABIN1 as a Regulator of NF- $\mathrm{B}$ Signaling ................................................. 14

1.5 - Discovering a Protective Role for ABIN1 in Glomerular Disease Pathogenesis 17

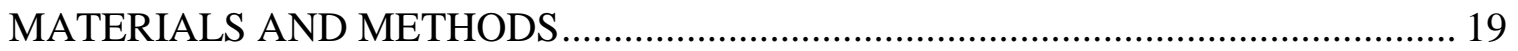

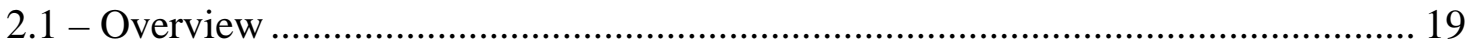

2.2 - Molecular Biology and Genetic Protocols .................................................. 19

2.3 - Plasmids and Lentiviral Transduction......................................................... 25

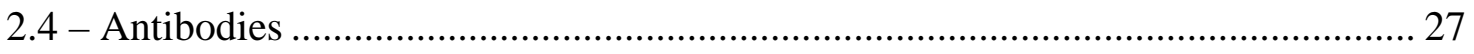

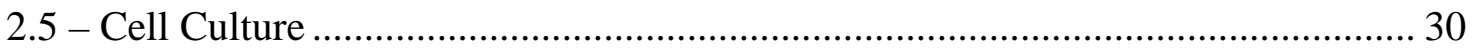


2.6 - General Protein Expression Assays.

2.7 - Mass Spectrometry Based Protein Detection 34

2.8 - Animal Handling and Assays for Primary Tissue. 35

PROTEOMIC APPLICATIONS FOR DEFINING CELLULAR MECHANISMS AND DIAGNOSTIC MARKERS OF SYSTEMIC LUPUS ERYTHEMATOSUS . 42

3.1 - Overview of mass spectrometry and discovery proteomics 42

3.2 - Cardiovascular systemic lupus erythematosus 47

3.4 - Lupus nephritis 53

3.5 - Neuropsychiatric systemic lupus erythematosus 57

3.6 - Other uses for proteomics in the study of SLE 63

3.7 - Characterization of the Secretome of Cultured Cells 65

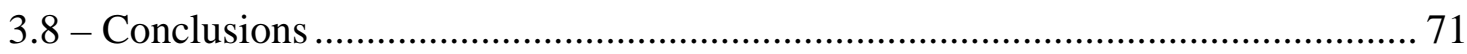

ABIN1 DYSFUNCTION AS A GENETIC BASIS FOR LUPUS NEPHRITIS .............. 74

4.1 - Introduction 74

4.2 - Results 75

4.3 - Discussion 88

PODOCYTE ABIN1 DETERMINES THE SEVERITY OF ANTIBODY-MEDIATED GLOMERULONEPHRITIS THROUGH A NOVEL PODOCYTE-NEUTROPHIL

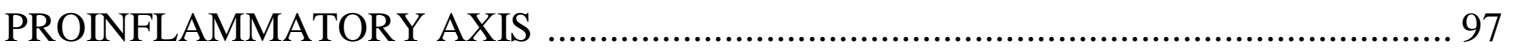

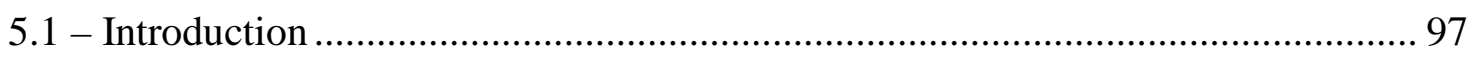


5.2 - Results .

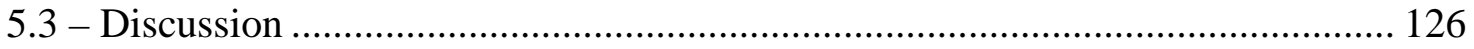

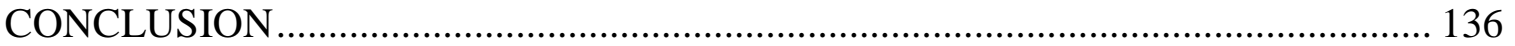

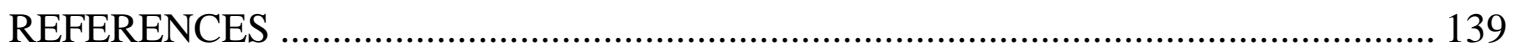

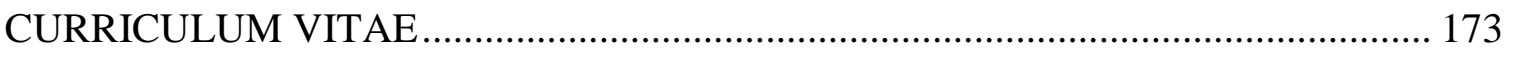




\section{LIST OF FIGURES}

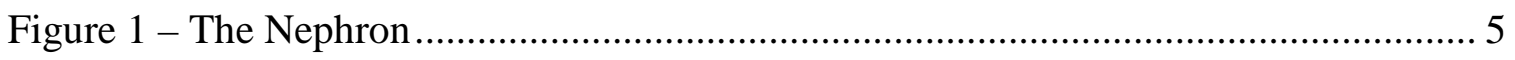

Figure 2 - Diagram of Glomerular Structures ...................................................... 7

Figure 3 - The Podocyte Slit Diaphragm Structures .................................................. 8

Figure 4 - Foot Process Pathologies Following Injury ............................................ 10

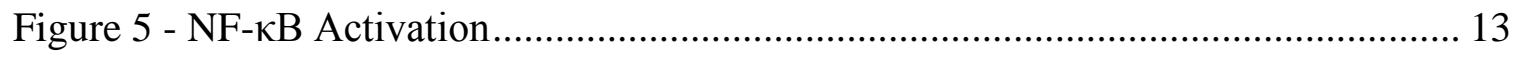

Figure 6 - ABIN1-mediated Inhibition of NF-кB Signaling ...................................... 16

Figure 7 - Flowchart of LC-MS/MS Sample Preparation............................................. 46

Figure 8 - Kinetics of Cytokine Secretion by Podocytes in Response to TNF- $\alpha$

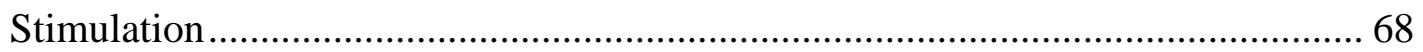

Figure 9 - Identification of Total Protein Secreted from Cultured Podocytes ................. 69

Figure 10 - Identification of Proteins Secreted by Podocytes Following 30 kDa Size-

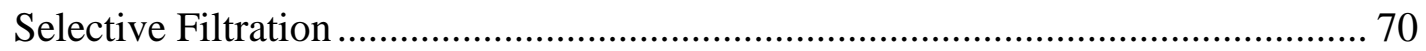

Figure 11 - ABIN1[D485N] Mice Develop Severe Progressive GN............................ 78

Figure 12 - Urine Albumin to Creatinine is Elevated in ABIN1[D485N] Mice.............. 80

Figure 13 - Complement Component 3 Levels are Reduced in Late Disease................. 81

Figure 14 - Renal Histology of WT and ABIN1[D485N] Mice .................................. 82

Figure 15 - ABIN1[D485N] Mice Exhibit Interstitial Inflammation ............................. 83

Figure 16 - ABIN1[D485N] Mice Exhibit Interstitial Fibrosis .................................... 84 
Figure 17 - ABIN1[D485N] mouse glomeruli display mesangial and subendothelial deposits. 85

Figure 18 - Deposits in ABIN1[D485N] glomeruli contain complement factors and immunoglobulins. 87

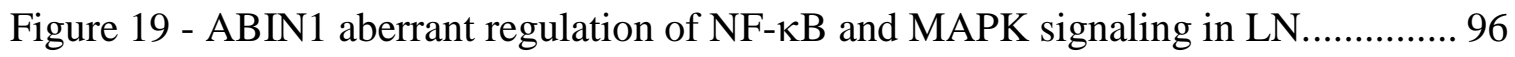

Figure 20 - ABIN1[D485N] Mice More Sensitive to NTS than WT............................ 100

Figure 21 - Bone Marrow Transplant Suggests Host Genotype is an Important Factor in Response to NTS 102

Figure 22 - Serum Cytokine Analysis of WT and ABIN1[D485N] Mice before and following NTS 103

Figure 23 - CD45-positive and MPO-positive Cell Influx into Glomeruli. 105

Figure 24 - Representative Images for Immune Cell Influx into Glomeruli Post-NTS.. 106

Figure 25 - Histological Analysis of PAS-stained Sections. 107

Figure 26 - Representative Images for Histological Analysis of Glomeruli Following NTS 108

Figure 27 - Ultrastructural Analysis of NTS-induced Changes to Glomerular Basement

Membrane 109

Figure 28 - WT-staining per Glomeruli. 111

Figure 29 - Representative Images for WT1 Staining 112

Figure 30 - Expression of Proinflammatory Markers from Isolated Glomeruli from WT and ABIN1[D485N] Mice 113

Figure 31 - Expression of Proinflammatory Factors in Cultured Podocytes. 115

Figure 32 - Single Target RT-qPCR Validation of Array Data 116 
Figure 33 - ELISA Quantification of Secreted Cytokines from Cultured Podocytes..... 117 Figure 34 - Induction of Neutrophil Chemotaxis by Podocyte Cell Culture Supernatant

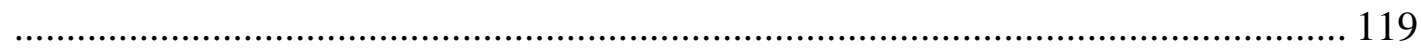

Figure 35 - Induction of Neutrophil Degranulation Markers by Podocyte Cell Culture

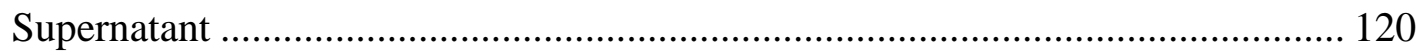




\section{LIST OF TABLES}

Table 1 - Contributions of Mass Spectrometry to Cardiovascular Biomarker Discovery in SLE 49

Table 2 - Contributions of Mass Spectrometry to Immunological Biomarker Discovery in

SLE 52

Table 3 - Contributions of Mass Spectrometry to Biomarker Discovery in Lupus

Nephritis 55

Table 4 - Contributions of Mass Spectrometry to Biomarker Discovery within Pediatric

Populations of Lupus Nephritis Patients.............................................................. 58

Table 5 - Contributions of Mass Spectrometry to Biomarker Discovery in

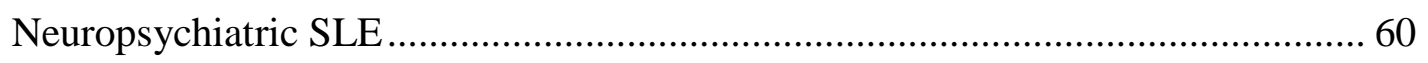

Table 6 - Glomerular, interstitial and tubular pathology assessment in wild-type (WT)

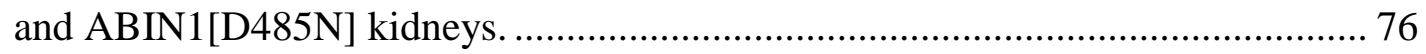

Table 7 - Sample summary before quality control adjustments. ....................................... 89

Table 8 - Sample Summary Following Quality Control Adjustments.............................. 90

Table 9 - The association results comparing SLE cases with lupus nephritis versus SLE cases without lupus nephritis. 


\section{CHAPTER I}

\section{BACKGROUND AND INTRODUCTION}

\section{$\underline{1.1-O v e r v i e w}$}

The basic role of the kidney is to provide selective filtration of blood for removal of wastes, homeostatic maintenance of vascular solutes, and regulation of blood volume. Together, the kidneys receive roughly $25 \%$ of cardiac output. This high rate of perfusion is required for optimal renal function and reduction in the glomerular perfusion results in reduced renal function. The renal arterial vasculature terminates at a small ball of capillaries termed the glomerulus which is where initial filtration takes place. Damage to the glomerulus results in protein and/or whole cell loss into the urine and reduced renal function [1]. Glomerulonephritis (GN) is a term which represents a heterogeneous group of diseases which accounts for $20 \%$ of chronic kidney diseases and is the leading cause of end-stage renal disease (ESRD) worldwide, although it ranks third in the United States behind diabetes and hypertension [2]. While GN is rather rare, unlike diabetes and hypertension, it predominantly affects young people and results in a lifelong medical burden for the patient and the health system. 
Many of the conditions clustered under the grand title of GN share a similar etiology, that is, deposition of antibodies within the glomerulus. These circulating antibodies may deposit in the glomerulus through passive means with low or no direct affinity to the glomerular structures, which is the case in Lupus Nephritis (LN) and IgA nephropathy[1,3,4]. In some cases, these antibodies are specific for glomerular structures, as in Goodpasture disease as well as membranous glomerulonephritis (formerly "Idiopathic" GN before identification of the Phospholipase A2 receptor as the autoantigenic target of membranous glomerulonephritis in our lab in 2009) [5-7].

It can be argued that the archetypical autoimmune condition throughout medical history is systemic lupus erythematosus (SLE), characterized by an abnormal immune response leading to loss of self-tolerance, autoantibody production, immune complex formation, $\mathrm{T}$ cell activation, and inflammatory cytokine release [8]. Medical historians note writings by Hippocrates that refer to the condition, but it was not until the late 1800's and early 1900's when it was described in the modern sense [9]. Multiple factors contribute to the immune response in SLE including genetic, epigenetic, immunoregulatory, environmental, and hormonal factors [8,9]. SLE has the ability to affect essentially any body tissue, including the kidney.

Lupus nephritis (LN) occurs clinically in about 50\% of patients with SLE and is a major cause of morbidity and mortality [10]. The incidence of LN is variable among different ethnic groups suggesting that genetic factors play a role in its pathogenesis. Primarily, individuals of African ancestry are at increased risk for LN [4]. Immunosuppressive treatment is effective in only about $50 \%$ of $\mathrm{LN}$ patients, and that therapy is associated with undesirable short- and long-term side effects [11-14]. Thus, 
identifying the molecular mechanisms responsible for the pathogenesis of LN is necessary to define more specific diagnostic and therapeutic targets. Such understanding may provide biomarkers of disease state which would enable treatment to be administered most effectively along with assessment of positive response to therapy such that the treatment can be modified or stopped in a timely manner to best manage adverse side effects.

These markers also provide important pathogenic insight and tools for testing new or improved therapeutics. Historically, SLE was diagnosed based solely on clinical features (i.e. rash, arthritis) and only recent times have seen the development of quantifiable diagnostic markers for laboratory confirmation of clinical findings by measurement of immune cell profiles and activity, identification of specific autoantibodies, and identification of changes in protein expression profiles in bodily fluid (i.e. urine, blood, cerebral spinal fluid) [9]. Traditional approaches to biomarker discovery allowed only limited numbers of identified proteins per sample which, unfortunately, required an initial selection bias on the part of the researcher. Recent improvements in mass spectrometry based approaches to novel biomarker discovery have allowed a level of insight not previously possible through the identification of thousands of proteins from individual samples. Mass spectrometry-based studies aimed at defining diagnostic markers for SLE complications by comparing protein expression in normal and diseased bodily fluids have used two basic approaches; 1) assessment of protein expression differences from stained 2D gels followed by MALDI-TOF/TOF identification of the proteins in differing spots or 2) direct assessment and comparison of protein expression from intrinsic LC-MS/MS data $[8,10]$. The total picture of SLE and 
LN diagnosis, however, is still incredibly complex and interactions of genetic risks, environmental factors, and molecular events that contribute to the development of LN are only beginning to be defined.

Finally, novel discoveries involving the molecular mechanisms behind LN pathogenesis, in particular the early events surrounding the inflammatory cascade which takes place following antibody deposition within the glomerulus, have the potential to contribute to the understanding of other antibody-mediated glomerulonephropathies. While outcomes and long term histological changes may differ due to systemic or local differences in these conditions, many share the same acute initiating event involving glomerular antibody deposition, and thus novel therapies directed at inhibiting pathogenic changes in this earliest stage of disease may find utility across the GN spectrum[1,3,5,7,12,15]. For a more complete understanding of the events following antibody deposition within the glomerulus, we must first discuss the cellular anatomy of the kidney and the current physiological understanding of the cell types involved in the glomerular response to damage.

\section{2 - Renal Anatomy and the Cellular Pathogenesis of Glomerular Inflammation}

The functional unit of the kidney is the nephron (Figure 1). The glomerular capillaries serve as a size- and charge-dependent filtration barrier and are contained within the Bowman's capsule which also includes non-capillary volume (Bowman's space) for collection of the ultrafiltrate upon exiting the glomerular capillaries. Upon 


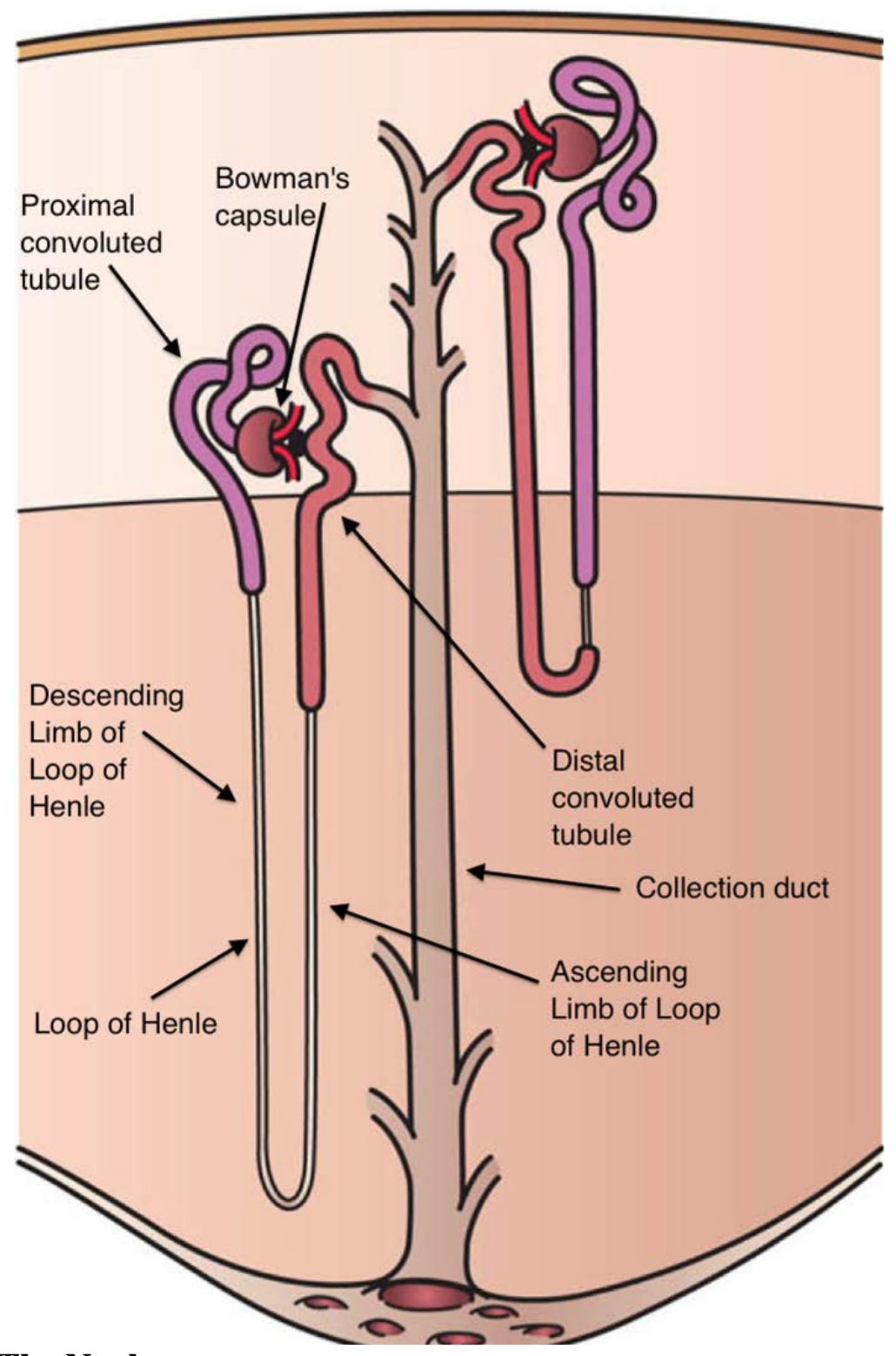

Figure 1 - The Nephron

The nephron is the functional unit of the kidney. The glomerular ball is held within the

Bowman's Capsule which drains into the proximal convoluted tubule, which begins the Loop of Henle, tasked with concentrating the urine before exit into the distal convoluted tubule and eventual draining into the collection duct which empties into the renal pelvis.

Copyright Permissions not required for this type of use.

http://open.umich.edu/education/med/resources/second-look-series/materials 
exiting the Bowman's space, the ultrafiltrate is concentrated and modified by cells lining the tubular walls. The direction of flow is as follows: the proximal convoluted tubule $\rightarrow$ descending limb of the loop of Henle $\rightarrow$ ascending limp of the loop of Henle $\rightarrow$ distal convoluted tubule $\rightarrow$ and finally the collecting duct which drains the concentrated urine into the renal pelvis. Pathologies have been reported at almost every point in the nephron and possess a multitude of instigating factors and as well as promising therapeutics [16]. However, the instigating factors in GN are almost invariably involving the glomerulus itself, which is where we now turn our focus.

Three cell types are predominant within the glomerulus: endothelial cells, mesangial cells, and podocytes. Figure 2 illustrates the arrangement of glomerular tissue. Endothelial cells line the endothelial side of the glomerular capillaries. Mesangial cells produce and maintain a significant portion of the extracellular matrix that supports the glomerulus. And finally, the epithelial podocytes are situated outside of the glomerular capillaries and critically support the glomerular capillary basement membrane. Podocytes accomplish this feat through cellular projections called foot processes, which interdigitate tightly to wrap the basement membrane. Small gaps between foot processes are called slit diaphragms and are very highly regulated by expression of membrane spanning proteins as well as intracellular structures and the actin cytoskeleton. Figure 3 diagrams this anatomy in a healthy glomerulus. Figure 3a shows regularly spaced foot processes with well-defined slit diaphragms separating them. Figure 3b describes a number of critical proteins involved in maintaining the slit diaphragm, namely nephrin and the underlying actin cytoskeleton itself. In pathologic conditions involving antibody deposition leading to inflammatory damage within the glomerulus, foot process structure 


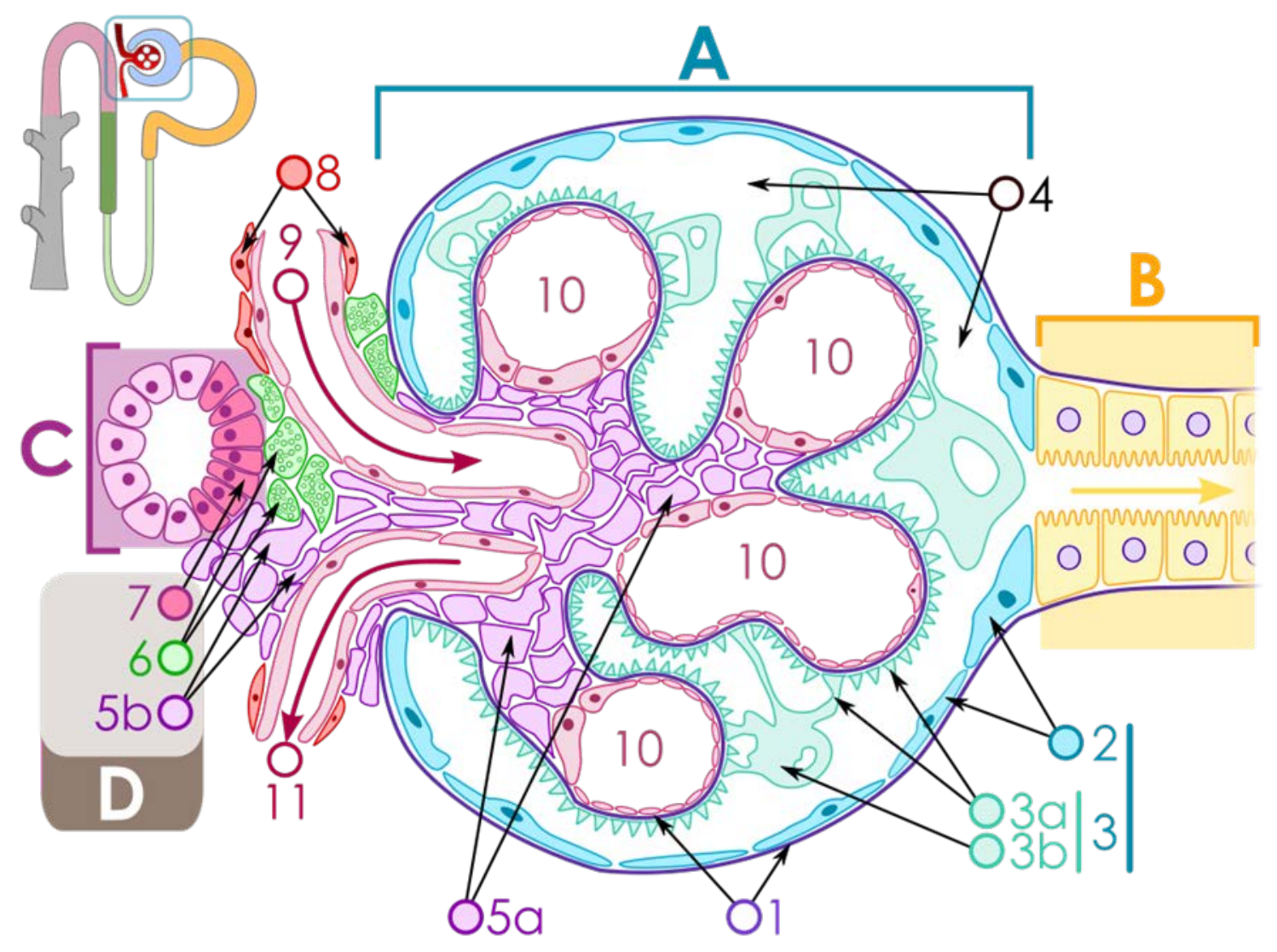

Figure 2 - Diagram of Glomerular Structures

A - Bowman’s Capsule, B - Proximal convoluted tubule, C - Distal convoluted tubule, D - Juxtaglomerular apparatus, 1. Basement membrane (Basal lamina), 2. Bowman's capsule - parietal layer, 3. Bowman's capsule - visceral layer, 3a. Pedicels (Foot processes from podocytes), 3b. Podocyte, 4. Bowman's space (urinary space), 5a. Mesangium - Intraglomerular cell, 5b. Mesangium - Extraglomerular cell, 6. Granular cells (Juxtaglomerular cells), 7. Macula densa, 8. Myocytes (smooth muscle), 9. Afferent arteriole, 10. Glomerulus Capillaries, 11. Efferent arteriole 


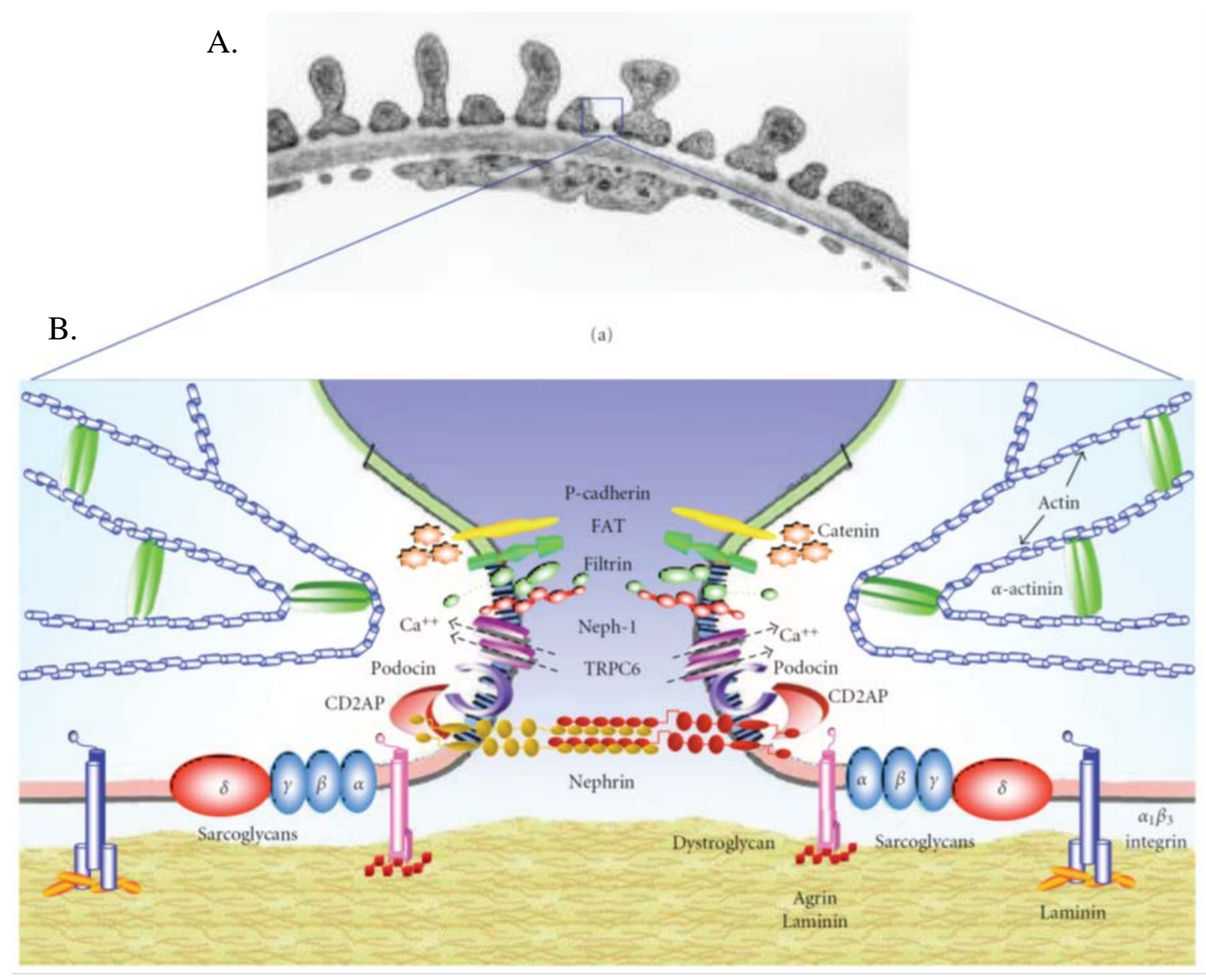

Figure 3 - The Podocyte Slit Diaphragm Structures

The podocyte slit diaphragm is an intensely maintained glomerular structure which is formed by the gap between podocytes foot processes. Loss of foot process structure is a common sign of glomerular pathology. (a) TEM image of cross section of GBM with slit diaphragm highlighted within the box. (b) Illustration of known slit diaphragm proteins. Of particular note in this figure is the association of f-actin of the podocyte cytoskeleton in the maintenance of the slit diaphragm.

Maddalena Gigante, Matteo Piemontese, Loreto Gesualdo, Achille Iolascon, and Filippo Aucella, "Molecular and Genetic Basis of Inherited Nephrotic Syndrome,” International Journal of Nephrology, vol. 2011, Article ID 792195, 15 pages, 2011. doi:10.4061/2011/792195

Copyright Permissions not required for this type of use 
changes as outlined in Figure 4. Regularly-spaced slit diaphragms on the epithelial side of the glomerulus are lost as foot processes efface, fuse, form pseudocysts, and potentially detach from the glomerular capillary completely [17].

It has been shown that mesangial cells respond to immunoglobulin, complement and cytokines by producing various cytokines and chemokines, resulting in increased endothelial cell expression of adhesion molecules that recruit and activate leukocytes $[15,18,19]$. Podocytes, too, are important factors in the glomerular inflammatory response, although they are markedly understudied. It is known that inflammatory injury leads to podocyte dysfunction, podocyte damage is a primary cause of proteinuria, and podocyte loss into the urine may be a better marker for glomerular injury than proteinuria $[17,20,21]$. Only a handful of published works have investigated the possibility of a direct role of podocytes in the induction of GN. Hewins et al. reported that IL-18 is expressed by podocytes in biopsies from patients with ANCA-associated vasculitis [22]. Kuravi et al. reported that TNF- $\alpha$ stimulated cultured podocytes to release IL-6, resulting in inhibition of leukocyte adhesion to vascular endothelial cells [23]. Yet, the response of podocytes to immunoglobulin and/or complement deposition and their contribution to the inflammatory response remain markedly understudied. These studies suggest that podocytes may participate in providing the cytokines and chemokines that control glomerular inflammation and further understanding of how the podocyte interacts with the immune system as well as the local tissue may provide pathogenic clues to more effective treatment or earlier diagnosis of poorly responding patients.

In contrast to the unknown role of podocytes, neutrophils have long been implicated in the pathogenesis of antibody-mediated GN, albeit recent studies tend to 


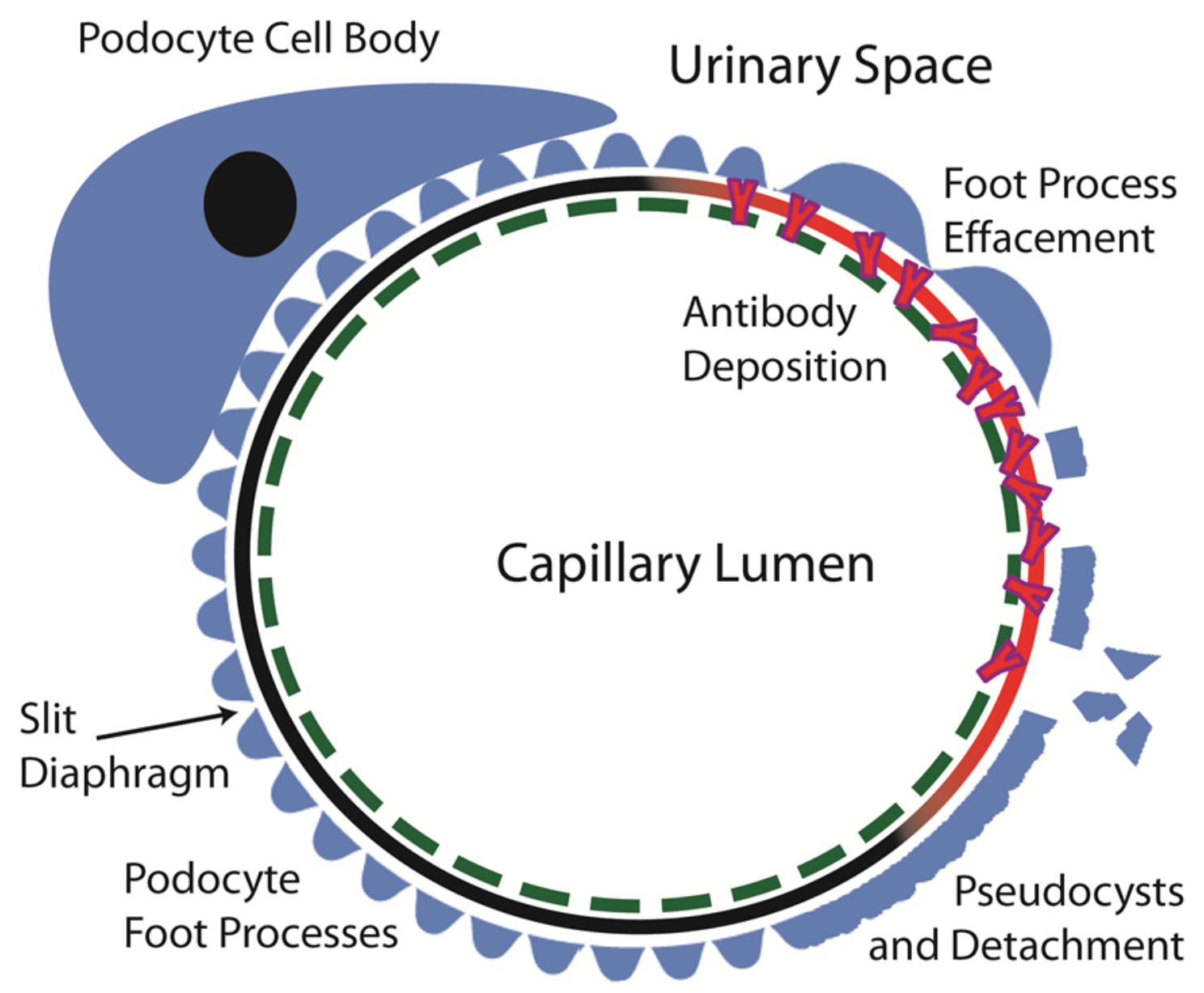

Figure 4 - Foot Process Pathologies Following Injury

The podocyte foot process is structurally sensitive to damage, and many insults can result in loss of foot process structure. Diseased glomeruli frequently exhibit foot process effacement, pseudocyst formation and bare areas of the GBM.

Illustration by Erik Korte, unpublished. 
focus primarily on the role of monocytes, B-cells, T-cells, and dendritic cells [23-29]. Neutrophils can release both reactive oxygen species and proteases contained in neutrophil granules have been reported to be responsible for degradation of the glomerular basement membrane and subsequent proteinuria [25-27]. Direct podocyte injury by neutrophils has not previously been evaluated. A recent report by Devi et al. showed that the glomerulus is a unique capillary bed that is constitutively patrolled by neutrophils which are retained within the glomerulus in the absence of proinflammatory stimulation [28]. Glomerular inflammation induced by deposition of anti-GBM antibodies increased the neutrophil retention time by 3- to 4-fold without increasing the number of neutrophils entering the glomerulus. Retention of neutrophils within the glomerulus, even in cases without proinflammatory stimulation, provides the opportunity for intimate interaction with local cells. Due to the prolonged retention of neutrophils in the glomerular capillaries, it is possible that small changes in localized expression of proinflammatory cytokines and chemokines may play a part in the induction of neutrophil activation in local tissues. Until this point, little has been known regarding the cytokine milieu secreted by podocytes and thus their role in disease pathogenesis. The work presented in this dissertation seeks to describe these unknown processes in renal pathophysiology.

\section{$\underline{1.3 \text { - Nuclear Factor kappa B in Inflammation and Disease }}$}


The transcription factor nuclear factor-kappa B (NF- $\mathrm{BB})$ regulates the expression of hundreds of genes that control cell proliferation and survival, the cellular stress

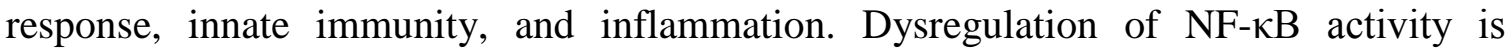
associated with many human diseases, especially those involving chronic inflammation, and recent studies suggest that NF-kB plays a role in the incidence and severity of GN as well [30-34]. In particular, both mesangial cells and podocytes demonstrate NF-kB activation and cytokine production in response to proinflammatory mediators [35-37]. Immunohistochemistry (IHC)-based studies have shown enhanced glomerular and tubular

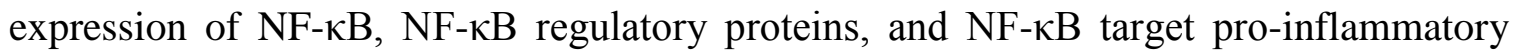
cytokines in renal biopsies from LN patients, compared with normal controls and patients with minimal change disease [33,34]. Another report found that pharmacological inhibition of NF-kB reduced the development of autoantibodies and renal impairment in SLE susceptible Fc $\gamma$ RIIb-deficient mice [30]. Treatment of spontaneous SLE developing SWRxNZB mice with a flavonoid, Apigenin, inhibited NF-kB mediated events in T cells and suppressed serum IgG levels resulting in delayed appearance of nephritis [31]. This inhibition of NF-kB signaling protects against development of disease, at least in part, through reduced expression of cytokines that are transcriptional targets of NF-kB (TNF$\alpha$, IL-1 $\beta$, IL-8, MCP-1, etc.) $[30,33,34,38]$.

NF- $\mathrm{kB}$ is activated by a variety of immune, inflammatory, and stress stimuli through many cytokine and toll-like receptors (TLR) and regulated through a complex interplay of proteins (recently reviewed in [39]). Figure 5 provides a general outline of the pathway. In resting cells, NF- $\mathrm{kB}$ is sequestered in the cytoplasm in an inactive state by binding to Inhibitor of $\kappa \mathrm{B}(\mathrm{I} \kappa \mathrm{B})$ proteins [40,41]. Following activation, the I $\kappa \mathrm{B}$ is 


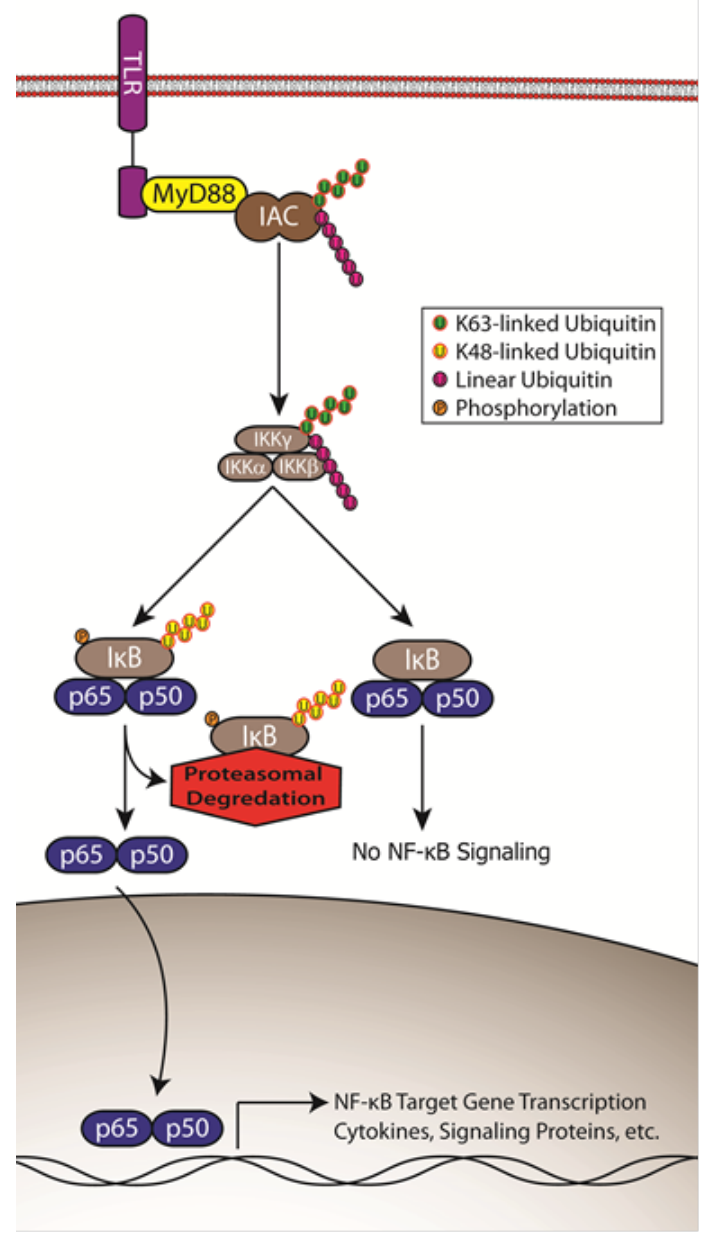

\section{Figure 5 - NF-кB Activation}

In the canonical NF- $\mathrm{BB}$ pathway, the transcription factor dimer is sequestered in the cytoplasm by $\mathrm{I} \kappa \mathrm{B}$. Phosphorylation and polyubiquitination of $\mathrm{I} \kappa \mathrm{B}$ results in its proteosomal degradation and translocation of NF- $\kappa \mathrm{B}$ to the nucleus. This is accomplished by an Inhibitor kappa Kinase (IKK) complex which includes an $\alpha, \beta$, and $\gamma$ subunit. The $\gamma$ subunit is called NEMO and is activated through linear or lysine-63 linked polyubiquitination by numerous upstream factors in the Inflammatory Activation Complex (IAC).

Illustration by Erik Korte, unpublished. 
phosphorylated, polyubiquinated through Lys48 linkages, and then degraded by the proteasome. This releases an active NF- $\mathrm{BB}$ complex to translocate to the nucleus and drive target gene expression. In the canonical NF- $\mathrm{KB}$ pathway, phosphorylation of IкB is mediated by the IкB kinase (IKK) complex, which consist of $\alpha, \beta$, and $\gamma$ subunits [42]. $\mathrm{IKK} \gamma$ is the regulatory subunit also referred to as NF-kB essential modulator (NEMO). Activation of IKK is mediated by an IKK Activating Complex (IAC), a diverse group of signaling proteins which includes TRAFs, RIPs, TAKs and IRAKs, and is assembled through protein-protein interactions to linear and lysine 63-linked and linear polyubiquitin chains and interacts with the linking protein MyD88 in TLR-mediated pathways [43-46]. NEMO binding to head-to-tail linked linear polyubiquitin chains or the Linear Ubiquitin Assembly Complex (LUBAC) also activates the canonical NF-kB pathway $[44,47,48]$. Tight regulatory control is required for NF- $\mathrm{\kappa B}$ signaling to prevent excessive production of many proinflammatory mediators.

\section{4 - ABIN1 as a Regulator of NF-кB Signaling}

The ubiquitin-binding protein, A20 binding inhibitor of NF-kB 1 (ABIN1), was first described in 1999 by Heyninck et al using a yeast two-hybrid screen and defined as a novel inhibitor of NF- $\mathrm{kB}$ and important A20 interacting partner [49]. She later described the structure and function of ABIN1, making note of the sequence homology of the ubiquitin bindings domains (UBD) of ABIN1 and NEMO [50]. Initial mouse models

reported conflicting results when ABIN1 expression was knocked out, resulting in 
embryonic lethality due to excessive liver apoptosis [51]. This embryonic lethality could be resolved through co-knockdown of the TNF- $\alpha$ receptor 1 alongside ABIN1, highlighting the importance of ABIN1 inhibition in the regulation of TNF- $\alpha$ induced signaling pathways [52]. Our lab returned to the original work of Heyninck et al to examine the homology between NEMO and ABIN1 as it pertains to autoimmunity and found that polyubiquitin binding to lysine 63-linked and linear polyubiquitin chains (as well as lysine 48-linked to a lesser degree) is required to prevent spontaneous immune hyperactivation in mice [53].

It is now clear that ABIN1 has important inhibitory roles for NF-kB signaling related to its ability to bind polyubiquitinated proteins and modulate their function [5255]. Figure 6 depicts ABIN1-mediated inhibition of a general NF-кB pathway. ABIN1 binds to lysine 63-linked and linear polyubiquitin chains and contains the same UBD as NEMO that facilitates binding to other regulatory proteins, such as TRAF2/6, RIP1, IRAK1 and others [47,52,53,56-58]. It is not clear how ABIN1 inhibits NF-кB activity, but two possible mechanisms have been proposed: the first is that ABIN1 binding competes with NEMO binding to proteins required for activation of IKK, and the second is that ABIN1 recruits A20 to the IKK regulatory complex where A20 disrupts the interactions needed for IKK activation by removing lysine 63-linked or linear polyubiquitin moieties from regulatory proteins [59,60]. A20 has also been reported to mediate proteasomal degradation of ubiquitin processing proteins that mediate IKK activation [61]. Asp (D) to Asn (N) mutation at residue 485 (mouse) or 472 (human) of the UBD renders ABIN1 incapable of binding polyubiquitin chains on NF-kB activators and is similar to the homologous [D311N] mutation in NEMO [44,50,51,53,62]. We 


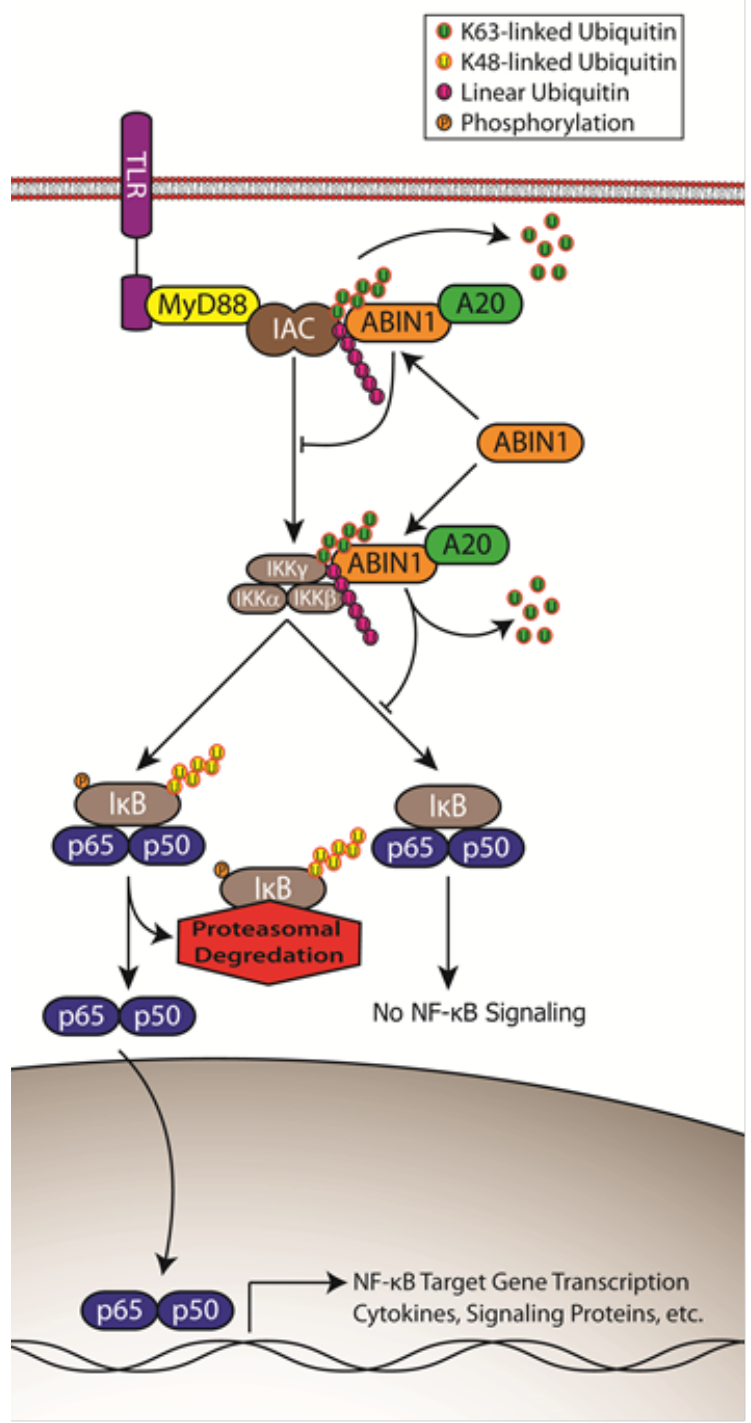

Figure 6 - ABIN1-mediated Inhibition of NF-אB Signaling

ABIN1 can bind polyubiquitinated substrates through its ubiquitin-binding domain (UBD). IKK $\gamma$ (NEMO) is activated through lysine-63 linked and linear polypolyubiquitin binding as are many factors in the IAC. ABIN1 binding may recruit A20 which can remove ubiquitin moieties from target proteins to inhibit their downstream functions.

Illustration by Erik Korte, unpublished. 
reported that a knock-in mouse in which wild type ABIN1 was replaced by ABIN1[D485N] induced constitutive activation of NF- $\kappa B$ signaling leading to elevated cytokine production and development of a progressive SLE-like autoimmune disease [53]. Renal injury in these animals was not characterized in this report.

\section{5 - Discovering a Protective Role for ABIN1 in Glomerular Disease Pathogenesis}

As outlined above, glomerular disease progression is highly associated with NF$\kappa \mathrm{B}$ activation and NF- $\mathrm{BB}$ expression in podocytes is also tied to potentially destructive phenotypes. Podocyte injury is made more severe by loss of nephrin in the slit diaphragm, which itself is known to inhibit NF- $\kappa B$ signaling, and NF- $\kappa B$ signaling in general has been shown to increase during progression to end-stage renal disease [63,64]. It has been reported that expression of NF- $\kappa \mathrm{B}$ proteins in podocytes was positively correlated to the severity of podocyte injury and subsequent proteinuria [33]. Genome wide association studies of American, European and Chinese SLE patients identified polymorphisms in ABIN1 which increased risk for developing disease [65-69]. What we currently know about ABIN1 suggests that it may have a role in the pathogenesis of glomerular disease. Therefore, we hypothesize that functional ABIN1 expressed in glomerular tissue, particularly in podocytes, is critical for maintenance of the glomerular microenvironment, control of local inflammation, and preservation of proper renal function.

This dissertation will present data showing that ABIN1 is, in fact, critical to 
proper glomerular inflammatory homeostasis. Novel mass spectrometry methods for characterization of ABIN1 phenotypic changes to podocytes will highlight the importance of proper NF-kB signaling regulation within podocytes. By using ubiquitin binding deficient ABIN1 in vivo and in vitro, the importance of functional ABIN1 to glomerular function will be described. Human polymorphisms in the ABIN1 locus will be defined as specific risk factors for development of glomerular disease. Pathological studies of tissue and cultured podocytes will define the role of ABIN1 in podocyte function. And a novel proinflammatory axis that exists between podocytes and neutrophils will be defined as a potential initiating step in the pathogenesis of antibodymediated GN. Finally, an inhibitor of neutrophil degranulation will be described with these models as a possible therapeutic treatment for early stages of glomerular disease. 


\section{CHAPTER II}

\section{MATERIALS AND METHODS}

\section{$\underline{2.1 \text { - Overview }}$}

Experimental characterization of intricate biological function requires numerous methods and rigorous, well-controlled experimental design. This presented a daunting challenge. I designed in vivo, in vitro, and ex vivo experiments capable of parsing the complex interplay of the immune system with a target tissue with a wide possibility of potential mechanisms, if any mechanism did in fact exist, to explain my hypothesis. My experimental design aimed to disprove my hypothesis and, failing that, shed light on the potential role of ABIN1 in the pathogenesis of glomerulonephritis.

\section{2 - Molecular Biology and Genetic Protocols}

Polymerase Chain Reaction - DNA amplification for cloning or analysis was performed on a CFX96 thermocycler (BioRad) or an EdvoCycler Model 541 (EdvoTek). 
AccuPrime PFX (Invitrogen) was the polymerase and standard PCR master mixes were used (Invitrogen). Reaction parameters were according to the manufacturer's specifications: 3 minutes at $95{ }^{\circ} \mathrm{C}$, followed by 30 cycles of $94{ }^{\circ} \mathrm{C}$ for 30 seconds, annealing at $50{ }^{\circ} \mathrm{C}$ for 30 seconds, extension at $72{ }^{\circ} \mathrm{C}$ for 30 seconds, with a final extension at $72{ }^{\circ} \mathrm{C}$ for 3 minutes.

Bacterial Transformation - One Shot TOP10 Chemically Competent E. coli (Invitrogen) were utilized for transformation of bacterial stocks. Stocks of chemically competent cells were mixed with $200 \mu \mathrm{g}$ plasmid DNA on ice for 30 minutes followed by a 30 second heat shock in a $42{ }^{\circ} \mathrm{C}$ water bath, then return to ice for 2 minutes. SOC media was added to the cells and they were incubated at $37^{\circ} \mathrm{C}$ for 1 hour. Transformed bacteria were then transferred to LB agar plates containing either Ampicillin $(100 \mu \mathrm{g} / \mathrm{mL})$ or Kanamycin (50 $\mu \mathrm{g} / \mathrm{mL}$ ) to match the resistance markers on the plasmid and incubated overnight at $37^{\circ} \mathrm{C}$. The following morning, single colonies were selected and grown in LB broth (Fisher Scientific) overnight at $37{ }^{\circ} \mathrm{C}$ with gentle agitation at 300 RPM. After $16-18$ hours, the bacteria were pelleted through centrifugation at $5000 \mathrm{x}$ g for 10 minutes and plasmid isolated as described below.

Plasmid Preparation - Plasmid preparation was accomplished using the Qiagen Mini Prep Kit or the Qiagen Maxi Prep Kit depending on the amount of plasmid needed (Qiagen, MD). The mini kit was sufficient for purification from 2-5 mL LB broth at high turbidity. The mega kit was sufficient for purification from up to $100 \mathrm{~mL}$ LB broth at high turbidity. 
Restriction Enzyme Digest - All restriction digests utilized in the course of this dissertation used enzymes from New England Biolabs which was also the source for all buffers and BSA (as required). Restriction digests were held at a static temperature (typically $37^{\circ} \mathrm{C}$ ) in an EdvoCycler (EdvoTek) if in a $50 \mu \mathrm{L}$ or smaller volume, or in a water bath calibrated to $37^{\circ} \mathrm{C}$ (Fisher Scientific).

Agarose Gel Electrophoresis - DNA amplicon, purified RNA or digested plasmid were run on $1 \%$ agarose gels to separate the DNA fragments by size using a $1 \mathrm{~kb}$ Plus DNA Ladder (Invitrogen) or a 10kb Plus DNA Ladder (Invitrogen) as appropriate. Agarose gels were produced fresh using $1 \mathrm{~g}$ powdered agarose (BioRad) per $100 \mathrm{~mL}$ 1x TAE buffer (BioRad). Standard gels comprised 50mL total volume and could be scaled up or down depending on specific needs of the sample. One volume of glycerol-based (5\% final concentration) DNA loading buffer was mixed 4 volumes of sample and current was applied at an appropriate level for an appropriate amount of time to achieve sufficient separation of the dye bands from the starting lane. Visualization of the DNA bands was achieved using Ethidium Bromide (Shelton Scientific) and UV excitation and digital image capture on the ChemiDoc MP (BioRad).

RNA Isolation -RNA was isolated from various tissues using the Aurum Total RNA Mini Kit (Qiagen). Appropriate volumes of starting samples were lysed with the lysis buffer, mixed 1:1 with RNA-grade Ethanol (Sigma) and bound to Aurum Mini Columns (Qiagen) for washing in proprietary buffers. Following the second wash, a 15 minute 
DNase protocol degraded genomic DNA, the final wash was applied, and purified RNA was isolated in RNase-free water. Quantification of RNA was achieved by readings in duplicate or triplicate on a NanoDrop 2000c spec (Thermo). All protocols using interaction with RNA required RNase-free materials, including tips, tubes and reagents. Great care was taken to prevent introduction of exogenous RNases from the environment. Many RNA samples were checked for sample integrity using gel electrophoresis and ethidium bromide staining as described above to assess a 2:1 ratio of staining for $28 \mathrm{~S}$ and $18 \mathrm{~S}$ bands, respectively, with properly sharp lines. This could be done post hoc and samples of RNA were always saved until completion of the experiment in case questions arose about sample integrity.

Reverse Transcription of cDNA - Purified RNA was reverse transcribed into cDNA using the iScript Reverse Transcription Supermix for RT-qPCR which employed a proprietary blend of oligo (dT) and random primers to provide unbiased representation of both 5' and 3' regions of transcript (BioRad). Optimal conditions allowed $1 \mu \mathrm{g}$ of template RNA in $16 \mu \mathrm{L}$ RNA eluate, combined with $4 \mu \mathrm{L} 5 \mathrm{x}$ Supermix, to allow a $20 \mu \mathrm{L}$ reaction volume as required. If RNA concentrations for the experiment were not sufficient in ALL samples to produce $1 \mu \mathrm{g}$ template RNA in $16 \mu \mathrm{L}$ reaction volume, all samples in the experiment were scaled down to a minimum input of 200 ng RNA per sample. This range was determined to be optimal in our lab for our transcript targets using our equipment and is not intended discount the much wider ranges published by BioRad. The $20 \mu \mathrm{L}$ final reaction volume could be scaled up to produce additional cDNA if experimental design called for more. Reverse transcription was performed on a CFX96 
thermocycler (BioRad) or an EdvoCycler Model 541 (EdvoTek). Each sample was mixed on ice and cycling parameters were as follows: priming at $25{ }^{\circ} \mathrm{C}$ for 5 minutes, reverse transcription at $42{ }^{\circ} \mathrm{C}$ for 30 minutes, and enzyme inactivation at $85{ }^{\circ} \mathrm{C}$ for 5 minutes, followed by a static hold at $4{ }^{\circ} \mathrm{C}$. cDNA was used immediately or stored at -20 ${ }^{\circ} \mathrm{C}$ with limited freeze/thaws in this final $\mathrm{RT}$ solution using the protocol volumes described below.

Primer Preparation - Primers for RT-qPCR were prepared using the database and software Primer-BLAST (NCBI) and selected custom primers were produced by Invitrogen (Carlsbad, CA). PCR product size was optimized for the transcript but generally was limited to 70-300 bp based on cycling parameters and Tm was optimized for each transcript as well. Multiple primer pairs were produced and tested for every transcript and for potential off target effects or primer dimers by melt-curve analysis on all analyzed samples. Primers which spanned introns were preferred to reduce interference of contaminate genomic DNA.

Real-Time Quantitative Polymerase Chain Reaction (RT-qPCR) - iTaq Universal SYBR Green Mastermix (BioRad) was used at $1 \mathrm{x}$ in a final reaction volume of $20 \mu \mathrm{L}$ per well of a 96-well RT-qPCR plate (BioRad). Each reaction included a final concentration of 0.4 $\mu \mathrm{M}$ forward and $0.4 \mu \mathrm{M}$ reverse primers optimized to be effective at that concentration for simplicity, and $1 \mu \mathrm{L}$ of output cDNA from the reverse transcription protocol. Only on rare instances were these parameters changed. SYBR green was used for its low cost and simplicity in primer design, although the potential for off target amplification required a 
melt curve analysis with each run. Thermocycling was performed on a CFX96 (BioRad). Following a 2 minute activation at $95{ }^{\circ} \mathrm{C}, 40$ cycles were completed of denaturation at 95 ${ }^{\circ} \mathrm{C}$ for 15 seconds followed by annealing and extension at $60{ }^{\circ} \mathrm{C}$ for 40 seconds. The melt curve analysis progressed from $65^{\circ} \mathrm{C}$ to $95^{\circ} \mathrm{C}$ in $0.5^{\circ} \mathrm{C}$ steps with 5 seconds per step. Housekeeping genes were optimized for each experiment but were typically either GAPDH or 18s rRNA, depending on stimulant and concentration of target. Fold changes were calculated using the $\Delta \Delta \mathrm{Ct}$ method.

Genome-Wide Association Study - A total of 16,999 independent case and control samples were collected from multiple sites as part of the Large Lupus Association Study 2 (LLAS2) with the Institutional Review Board (IRB) approval from each institution and processed at the Oklahoma Medical Research Foundation (OMRF) under the auspices of the OMRF institutional review board. Only individuals who signed informed consent forms were included in the study. All SLE cases met the revised 1997 American College of Rheumatology for classification of SLE. Among SLE cases, those with lupus nephritis fulfilled the renal criterion of either (1) persistent proteinuria $>0.5 \mathrm{~g}$ per day $(24 \mathrm{~h})$ or persistent $>3+$ if quantification was not performed or (2) presence of urinary cellular casts (Table 2). All SLE cases met the revised 1997 American College of Rheumatology for classification of SLE [70]. Among SLE cases, those with lupus nephritis fulfilled the renal criterion of either (1) persistent proteinuria $>0.5 \mathrm{~g}$ per day $(24 \mathrm{~h})$ or persistent $>3+$ if quantification was not performed or (2) presence of urinary cellular casts [70]. The custom-design Illumina iSelect platform at OMRF was used to genotype 5 SNPs in TNIP1 previously reported to be associated with systemic sclerosis (rs4958881, 
rs3792783, rs2233287) [71], SLE (rs7708392) [67], psoriasis (rs3762999, rs999556, rs17728338) [72,73], and psoriatic arthritis (rs17728338, one of psoriasis SNPs) [74]. In addition, 347 ancestral-informative markers (AIMs) spanning the genome were genotyped. After application of quality controls by excluding samples if they exhibited low call rates $(<90 \%)$ and extreme heterozygosity ( $>5$ standard deviations from the mean), revealed discrepancies between reported gender and genetic data, or were determined to be a duplicate or cryptic related to another sample (the proportion of alleles shared identical by descent $>0.4$ ), and removing extreme population outliers based on global ancestry estimation and principal component analysis (calculated using the AIMs in the ADMIXMAP[75,76] and EIGENSTRAT[77] programs, respectively) as described in other LLAS2 reports [66,78], a final dataset of 15,864 unrelated subjects was obtained. Single marker association analyses were calculated using the logistic regression function in PLINK v1.07 [79]. The additive genetic model was applied while adjusting for gender and global ancestry estimates (African, European, and East Asian) [66,80,81]. The Bonferroni corrected p-value threshold was set to $P<0.01$ based on multiple tests of 5 SNPs.

\section{$\underline{2.3 \text { - Plasmids and Lentiviral Transduction }}$}

pCMV6-AC-mGFP - This cloning system was purchased from Origene to produce GFPtagged ABIN1 in cultured cells at a higher level than endogenous ABIN1 was produced. A cytomegalovirus (CMV) promoter on the cassette accomplished this in all cell types 
used. This plasmid also conferred ampicillin resistance for selection and high level production in bacterial cultures. An insert sequence was produced and sent to Origene for production. Plasmid sequences were confirmed before being used and both GFP and ABIN1 proteins were recognized appropriately by all methods employed in the course of this study.

pCMV6-AC-DDK - This cloning system was produced as the GFP tagged system above, but rather than GFP, it included a DDK tail (non-proprietary FLAG system) for protein purification.

Lentiviral Transduction using MDM00734 pCSC-SP-PW - Some cells are resistant to transient transfection due to sensitivity to transfective agents or other reasons, thus Lentiviral transduction was used as an alternative, which produces stable expression of target proteins in difficult to transfect cells. This plasmid was designed by Michael Mendenhall of the University of Kentucky Viral Core. With the help of Dr. Mendenhall, we designed a protocol for isolating ABIN1 inserts of the appropriate genotype (WT and ABIN1[D472N] and others) from transfection plasmids and production of lentivirus capable of transduction of cultured podocytes. Per the University of Louisville IRB Approval, no virus was reproduced in University of Louisville facilities. All local viral stocks were replication deficient. An optimized multiplicity of infection (MOI) of 1-10 based on viral titer was used for each cell type and optimized to achieve equal expression of WT, ABIN1[D472N] or other genotype. Lentiviral stocks were aliquoted, frozen for transport and thawed only once at $37^{\circ} \mathrm{C}$ on the day of use. Cells grown in 6-well dishes 
(TTP) in $2 \mathrm{~mL}$ appropriate cell culture media were exposed to an appropriate volume of lentivirus in polybrene at $8 \mu \mathrm{g} / \mathrm{mL}$ (a kind gift of Dr. Mendenhall). Following an 8 hour incubation, media was removed and a second volume of $2 \mathrm{~mL}$ media with virus and 8 $\mu \mathrm{g} / \mathrm{mL}$ polybrene was added and allowed to infect overnight. Fresh media was applied and cells were allowed to grow for 72 hours before transduction could be checked via fluorescence microscopy on an inverted CKX41 (Olympus). Expression was confirmed via RT-qPCR, sequencing, western blot and other methods.

\section{$\underline{2.4-\text { Antibodies }}$}

ABIN1 - For detection of ABIN1, a mouse monoclonal antibody (Santa Cruz - sc376999) was used at 1:200 dilution (200 $\mu \mathrm{g} / \mathrm{mL}$ stock) in 5\% milk in 1x TTBS overnight at 4 degrees followed by goat-anti-mouse-HRP (Santa Cruz - sc-2005) at 1:2000 (200 $\mu \mathrm{g} / \mathrm{mL}$ stock) for 1 hour at room temperature and chemiluminescent using Clarity Western ECL Substrate (BioRad) on the BioRad ChemiDoc MP Image Station and ImageLab 3.0 Software (Biorad).

MCP-1/CCL2 - For detection of MCP-1, a mouse monoclonal antibody (Santa Cruz - sc377082) was used at 1:200 dilution (200 $\mu \mathrm{g} / \mathrm{mL}$ stock) in $5 \%$ milk in 1x TTBS overnight at 4 degrees followed by goat-anti-mouse-HRP (Santa Cruz - sc-2005) at 1:2000 (200 $\mu \mathrm{g} / \mathrm{mL}$ stock) for 1 hour at room temperature and chemiluminescent using Clarity 
Western ECL Substrate (BioRad) on the BioRad ChemiDoc MP Image Station and ImageLab 3.0 Software (Biorad).

GAPDH - For detection of GAPDH, a rabbit polyclonal antibody (Santa Cruz - sc25778) was used at 1:200 dilution (200 $\mu \mathrm{g} / \mathrm{mL}$ stock) in $5 \%$ milk in $1 \mathrm{x}$ TTBS overnight at 4 degrees followed by goat-anti-rabbit-HRP (Santa Cruz - sc-2004) at 1:2000 (200 $\mu \mathrm{g} / \mathrm{mL}$ stock) for 1 hour at room temperature and chemiluminescent using Clarity Western ECL Substrate (BioRad) on the BioRad ChemiDoc MP Image Station and ImageLab 3.0 Software (Biorad).

ET-1 - For detection of Endothelin 1, a goat polyclonal antibody (Santa Cruz - sc-21625) was used at 1:200 dilution $(200 \mu \mathrm{g} / \mathrm{mL}$ stock) in 5\% milk in $1 \mathrm{x}$ TTBS overnight at 4 degrees followed by rabbit-anti-goat -HRP (Santa Cruz - sc-2768) at 1:2000 (200 $\mu \mathrm{g} / \mathrm{mL}$ stock) for 1 hour at room temperature and chemiluminescent using Clarity Western ECL Substrate (BioRad) on the BioRad ChemiDoc MP Image Station and ImageLab 3.0 Software (Biorad).

ERK 1 - For detection of ERK 1, a rabbit polyclonal antibody (Santa Cruz - sc-94) was used at 1:200 dilution (200 $\mu \mathrm{g} / \mathrm{mL}$ stock) in 5\% milk in 1x TTBS overnight at 4 degrees followed by goat-anti-rabbit-HRP (Santa Cruz - sc-2004) at 1:2000 (200 $\mu \mathrm{g} / \mathrm{mL}$ stock) for 1 hour at room temperature and chemiluminescent using Clarity Western ECL Substrate (BioRad) on the BioRad ChemiDoc MP Image Station and ImageLab 3.0 Software (Biorad). 
$I \kappa B \alpha$ - For detection of $I \kappa \mathrm{B} \alpha$, a rabbit polyclonal antibody (Santa Cruz - sc-847) was used at 1:200 dilution (200 $\mu \mathrm{g} / \mathrm{mL}$ stock) in 5\% milk in 1x TTBS overnight at 4 degrees followed by goat-anti-rabbit-HRP (Santa Cruz - sc-2004) at 1:2000 (200 $\mu \mathrm{g} / \mathrm{mL}$ stock) for 1 hour at room temperature and chemiluminescent using Clarity Western ECL Substrate (BioRad) on the BioRad ChemiDoc MP Image Station and ImageLab 3.0 Software (Biorad).

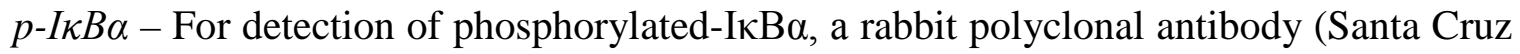
- sc-101713) was used at 1:200 dilution $(200 \mu \mathrm{g} / \mathrm{mL}$ stock) in 5\% milk in $1 \mathrm{x}$ TTBS overnight at 4 degrees followed by goat-anti-rabbit-HRP (Santa Cruz - sc-2004) at 1:2000 (200 $\mu \mathrm{g} / \mathrm{mL}$ stock) for 1 hour at room temperature and chemiluminescent using Clarity Western ECL Substrate (BioRad) on the BioRad ChemiDoc MP Image Station and ImageLab 3.0 Software (Biorad).

Podocin - For detection of podocin, a rabbit polyclonal antibody (Santa Cruz - sc-21009) was used at 1:200 dilution $(200 \mu \mathrm{g} / \mathrm{mL}$ stock) in 5\% milk in $1 \mathrm{x}$ TTBS overnight at 4 degrees followed by goat-anti-rabbit-HRP (Santa Cruz - sc-2004) at 1:2000 $(200 \mu \mathrm{g} / \mathrm{mL}$ stock) for 1 hour at room temperature and chemiluminescent using Clarity Western ECL Substrate (BioRad) on the BioRad ChemiDoc MP Image Station and ImageLab 3.0 Software (Biorad).

p-ERK - For detection of phosphorylated ERK, a mouse monoclonal antibody (Santa Cruz - sc-7383) was used at 1:100 dilution (200 $\mu \mathrm{g} / \mathrm{mL}$ stock) in $5 \%$ milk in $1 \mathrm{x}$ TTBS 
overnight at 4 degrees followed by goat-anti-mouse-HRP (Santa Cruz - sc-2005) at 1:2000 (200 $\mu \mathrm{g} / \mathrm{mL}$ stock) for 1 hour at room temperature and chemiluminescent using Clarity Western ECL Substrate (BioRad) on the BioRad ChemiDoc MP Image Station and ImageLab 3.0 Software (Biorad).

A20 - For detection of A20, a mouse monoclonal antibody (Santa Cruz - sc-376564) was used at 1:100 dilution (200 $\mu \mathrm{g} / \mathrm{mL}$ stock) in 5\% milk in 1x TTBS overnight at 4 degrees followed by goat-anti-mouse-HRP (Santa Cruz - sc-2005) at 1:2000 (200 $\mu \mathrm{g} / \mathrm{mL}$ stock) for 1 hour at room temperature and chemiluminescent using Clarity Western ECL Substrate (BioRad) on the BioRad ChemiDoc MP Image Station and ImageLab 3.0 Software (Biorad).

$\beta$-actin - For detection of $\beta$-actin for use as a loading control, a mouse monoclonal antibody conjugated to HRP (Santa Cruz - sc-47778-HRP) was used at 1:500 dilution (200 $\mu \mathrm{g} / \mathrm{mL}$ stock) in 5\% milk in $1 \mathrm{x}$ TTBS for 1 hour at room temperature and chemiluminescent using Clarity Western ECL Substrate (BioRad) on the BioRad ChemiDoc MP Image Station and ImageLab 3.0 Software (Biorad).

\section{$\underline{2.5-\text { Cell Culture }}$}

Conditionally Immortalized Podocytes - In 2002, Moin Saleem published his characterization of a conditionally immortalized podocyte cell line using a temperature 
sensitive SV40-T-gene that revolutionized almost all modern research into podocyte biology [82]. Briefly, these cells grow permissively at $33^{\circ} \mathrm{C}$, but upon exposure to $37^{\circ} \mathrm{C}$ temperatures for 7-10 days, they enter growth arrest and express markers of differentiated podocytes in vivo. These cells were grown in RPMI-1640 without glutamine (Invitrogen), supplemented with 1x Pen/Strep/Glutamine (Invitrogen), 10\% fetal bovine serum (Sigma), and 1x Insulin/Transferrin/Selenium-X (Invitrogen). Many stimulants were used, but this dissertation focuses on TNF- $\alpha(100 \mathrm{ng} / \mathrm{mL})($ BioLegend) for all cell culture work.

Podocyte Actin Labeling and Confocal Microscopy - Confocal microscopy images were obtained as previously described[83]. Briefly, 35mm collagen-coated glass bottom dishes (MatTek) were seeded with podocytes and allowed to mature at $37^{\circ} \mathrm{C}$ for 10 days. Cells were serum starved with $0.5 \%$ FBS medium $24 \mathrm{~h}$ before stimulant was added. Following stimulation, cells were rinsed three times with PBS that contained calcium and magnesium and fixed in 3.7\% paraformaldehyde (Azer Scientific) in PBS (Invitrogen) for 10 min, followed by permeabilization with $0.025 \%$ NP-40 (Sigma) in PBS for 15 min and washing. Cells were blocked in 5\% BSA (Sigma) in PBS for 30 minutes before addition of $200 \mu \mathrm{L}$ (3 units) rhodamine-phalloidin (Thermo) for 30 minutes. Following another wash, $300 \mathrm{nM}$ DAPI (Invitrogen) was incubated for $5 \mathrm{~min}$, and rinsed three times with PBS, mounted using Vectashield Antifade Mounting Medium for Fluorescence (Vector Labs). Images were acquired using a Zeiss confocal microscope and analyzed using LSM510 software. The images for rhodamine were analyzed by FibrilTool as recommended by the published protocol[84]. 


\section{$\underline{2.6 \text { - General Protein Expression Assays }}$}

Sample Preparation and Cellular Lysis - Cultured cells were washed 3 times with icecold PBS and lysed in RIPA Buffer (Sigma) containing 1x Protease Inhibitor Cocktail (Sigma) and 1x Phosphatase Inhibitor Cocktail (Santa Cruz). Lysis was allowed to proceed on ice for 30 minutes followed by a centrifugation at 20,000 $\mathrm{x}$ g for 30 minutes at $4{ }^{\circ} \mathrm{C}$ to pellet cellular debris. An appropriate volume of lysis buffer was used to accommodate either the confluency of cells on the plate or the approximate size of the cell pellet.

Protein Quantification - Protein concentration was estimated using the BCA method (Thermo) in triplicate according to the manufacturer's protocol. Lysis buffer or vehicle into which the sample is diluted was used as a blank and serial dilutions of BCA (Thermo) were used to generate a standard curve.

SDS-PAGE - Appropriate volumes of protein sample and water were added to $5 \mathrm{x}$ Laemmli Sample Buffer (final concentration $60 \mathrm{mM}$ Tris-Cl pH 6.8, 2\% SDS, 10\% glycerol, $5 \% \beta$-mercaptoethanol, $0.01 \%$ bromophenol blue) to achieve equal estimated loading of all samples to be loaded in the gel. Mini-PROTEAN TGX pre-cast 4-20\% gradient gels (BioRad) were immersed in 1x Tris-Glycine-SDS buffer (BioRad) and run under constant voltage (15-250V) deemed appropriate for desired time of separation or 
size of target proteins. Precision Plus Dual Color protein standard (BioRad) was used to gauge size of bands identified in western blot protocol (in $\mathrm{kDa}$ ).

Polyvinylidene Fluoride (PVDF) Transfer - PVDF (BioRad) was used rather than nitrocellulose. PVDF was rehydrated for at least one minute in 100\% methanol (Sigma) followed by at least 10 minutes in 1x Tris-Glycine transfer buffer (BioRad). Semi-dry transfer was achieved using the Trans-Blot Turbo (BioRad) at appropriate current (usually $300 \mathrm{~mA}$ but varied based on sample). Totality of transfer was assessed by staining the gel post-transfer with Coomassie stain for 15 minutes followed by 1-4 hours of destain in $40 \%$ methanol (Sigma) and 10\% acetic acid (Sigma).

Western Blotting - PVDF membrane was washed 3 times in 1x TTBS followed by >30 minute block in 5\% milk (Great Value) in TTBS. Appripriate dilutions of primary and secondary antibodies were added to 5\% milk in TTBS. Primary antibodies were allowed to bind overnight at $4{ }^{\circ} \mathrm{C}$ with rocking, followed by 3 washes in $1 \mathrm{x}$ TTBS. HRPconjugated secondary antibodies were allowed to bind for 1 hour at room temperature with gentle rocking before washing 3 times in 1x TTBS. Chemiluminescent detection was achieved using Clarity Western ECL Substrate (BioRad) on the BioRad ChemiDoc MP Image Station and ImageLab 3.0 Software (Biorad).

Enzyme-Linked Immunosorbent Assay (ELISA) - Secreted cytokine levels for MCP-1, IL8, and TNF- $\alpha$ were analyzed using the BioLegend ELISA Max Deluxe kits per the manufacturer protocol. To allow secretome samples to be utilized with primary 
neutrophils, samples were stimulated for 6 hours with TNF- $\alpha$ followed by 3 washes in sterile, endotoxin-free Krebs buffer, followed by a 6 hour collection in 10mL Krebs buffer per $10 \mathrm{~cm}$ dish at $70 \%$ confluence. This stimulation and wash regimen was determined to be optimal for the cytokines of interest and no detectable TNF- $\alpha$ remained following the 3 wash steps $(<<2 \mathrm{pg} / \mathrm{mL})$.

Luminex-based Cytokine Arrays - Blood was collected from mice by cardiac puncture, allowed to clot, and the serum was separated by centrifugation. Cytokines in mouse serum were measured using the Bio-Plex Mouse Cytokine 23-plex assay on the Bio-Plex MAGPIX Multiplex reader according to the manufacturer’s protocol (Bio-Rad).

\section{$\underline{\text { 2.7 - Mass Spectrometry Based Protein Detection }}$}

Label-free Sequencing and Quantification of Total Protein - Briefly, protein concentration was estimated using the BCA method (Thermo, Rockford, IL), diluted with 6M urea in $100 \mathrm{mM}$ ammonium bicarbonate, reduced with dithiothreitol and alkylated with iodoacetamide. The sample was digested using sequence-grade trypsin at a 1:100 ratio of trypsin to sample protein and incubated overnight at $37{ }^{\circ} \mathrm{C}$ with shaking, then desalted and concentrated using a fused silica microcapillary C-18 column (Michrom Bioresources, Auburn, CA). The resulting peptides were analyzed by a Thermo Fisher Scientific LTQ-Orbitrap Elite mass spectrometer coupled with a Proxeon Easy nanoLC 1000 UHPLC system (Thermo, Rockford, IL). Protein identification was performed with 
Sequest Sorcerer (Sage-N Research, San Jose, CA), set up to search a FASTA formatted human protein database (Human RefSeq). High-probability peptide and protein assignments were made using Peptide and Protein Prophet algorithms[85]. Abundance of each identified protein was determined by normalizing the number of unique spectral counts matching to the protein by its predicted molecular weight, termed the protein abundance factor [5,86,87]. Cytokine production by unstimulated ABIN1 WTexpressing podocytes was defined as the normal cytokine secretome and was compared to the ABIN1 WT stimulated and both stimulated and non-stimulated ABIN1[D472N] dataset to identify changes to the normal secretome of these cells.

Secretome Identification, Size Selective - Most cytokines are small proteins and we have found that sample purity is increased through a 10 minute centrifugation in an Amicon Ultra-30kDa Centrifugal Filter Unit (Millipore, Billerica, MA) to remove proteins larger than $30 \mathrm{kDa}$, followed by preparation as we have previously described.

\section{$\underline{2.8 \text { - Animal Handling and Assays for Primary Tissue }}$}

Transgenic Mice - The ABIN1[D485N] global knockin mice were generated on a 129SvJxC57BL/6 background[53] and then backcrossed eight generations to C57BL/6 for the present studies. Heterozygous mice were used to generate ABIN1[D485N] homozygous and littermate WT control mice for all the experiments. Female mice from 0-6 months of age were utilized as indicated. All animals were maintained in specific 
pathogen free conditions and all studies were approved by the Institutional Animal Care and Use Committee of the University of Louisville (Louisville, KY).

Bone Marrow Transplant and Development of Chimeric Animals - Bone marrow transplant was carried out as previously described[88]. Briefly, 5-7 week old female mice were conditioned with 950 cGy total body irradiation from a caesium source (Gamma-cell 40; Nordion, Ontario, Canada). At 24 h post irradiation, the mice were transplanted with bone marrow cells $\left(1 \times 10^{7}\right.$ cells per mouse in $\left.0.1 \mathrm{ml} \mathrm{PBS}\right)$ isolated from tibias and femurs of age-matched donor mice, through the retro-orbital plexus with a 27-gauge needle. Ten WT mice received WT bone marrow and ten received ABIN1[D485N] bone marrow. Likewise, ten ABIN1[D485N] mice received WT bone marrow and ten received ABIN1[D485N] bone marrow. Engraftment was allowed to proceed for 5-7 weeks before administration of $\alpha$-GBM. Engraftment was confirmed using leukocytes isolated from whole blood. Both the recipient and donor mice were maintained on normal chow throughout the experiment.

Urine Albumin:Creatinine Measurements - Spot urine samples were captured by scruffing the mice and massaging the bladder over a clean, disposable surface and collected in a sterile microcentrifuge tube. Urine was centrifuged at $5000 \mathrm{x} \mathrm{g}$ for 5 minutes then standard sandwich ELISA was performed in triplicate according to the manufacturer's protocol for mouse albumin (Bethyl) and a kinetic Jaffe method for creatinine (Thermo). The ratio of albumin $(\mu \mathrm{g} / \mathrm{mL})$ and creatinine $(\mathrm{mg} / \mathrm{mL})$ was calculated for each sample at each time point. 
Serum C3 Quantification - Serum was collected from ABIN1[D485N] and WT mice at ages 3-4 months and 5-6 months. C3 measurements were performed using a 1:25,000 dilution of mouse serum and measurement with the Mouse C3 ELISA Kit from GenWay Biotech (\#40-374-130047) per the manufacturer’s guidelines. All samples were tested in duplicate and the average was reported.

Histopathology and immunohistochemical staining of mouse cortical tissue - Formalinfixed, paraffin embedded tissues were stained with PAS reagent for histological analysis by light microscopy. Glomerular damage was assessed by semiquantitative scoring of cellularity and mesangial matrix expansion on a 0 (normal), 1+ (mild abnormality), 2+ (moderate abnormality), and 3+ (severe abnormality) scale. For immunohistochemical labeling of proteins, sections were deparaffinized in xylene and rehydrated in graded alcohols. Antigen retrieval was achieved using citrate buffer solution for 20 minutes at $95^{\circ} \mathrm{C}$ (Dako), slides were washed in buffer and endogenous peroxidases were quenched with $\mathrm{H}_{2} \mathrm{O}_{2}$ for 5 minutes before washing and a 30 minute block in buffer containing 5\% BSA and $2 \%$ sera at RT. Primary antibody was applied overnight at $4{ }^{\circ} \mathrm{C}$ and $1: 200$ dilution for WT-1 (Santa Cruz), CD45 (Angio-Proteomie), 1:50 dilution for MPO (AbCam) or with buffer only as a negative control followed by washing and incubation with appropriate secondaries at 1:200 dilution. All sections including negative controls were then incubated with respective biotinylated secondary antibodies (1:200, Vector Labs), followed by incubation in avidin/biotinylated enzyme complex (Vectastain Elite ABC kit, Vector Labs). Proteins were detected following color development using 3,3'- 
diaminobenzidine (DAB) as substrate (Vector Labs). Counterstaining was performing using hematoxylin (Vector Labs) and cells were dehydrated, mounted and viewed at 100x. Digital Images were obtained with a Q Color 5 camera attached to an Olympus BX51 microscope using Image-Pro software.

Immunofluorescence - For immunofluorescent analysis of C3, IgA or IgM, 4 micron frozen sections were first washed in PBS 3x5 minutes each. The blocking solution was applied for 30 minutes at room temperature. The primary antibody was then added for 2 hours at room temperature. The sections were washed for 5 minutes in PBS three times. FITC primary antibody (C3- Cedar Lanes Laboratory, IgG- Sigma Aldrich, C1q- Hycult Biotech) or unlabeled primary antibody (IgA-Invitrogen, IgM-Invitrogen) followed by florescent secondary antibody (Alexa Flour 555- Invitrogen) were used. Following antibody incubation, slides were washed in PBS 3x5 minutes. Images were acquired using a Zeiss confocal microscope and analyzed with LSM510 software.

Transmission Electron Miscroscopy - Freshly dissected cortical tissue was placed in a cryomold with OCT compound (Tissue-Tek) and flash frozen in liquid nitrogen. Thick (1-mm) sections were prepared to evaluate the orientation and presence of glomeruli. Thin sections were mounted on 200 mesh naked copper grids and stained with lead citrate and uranyl acetate (4\% in absolute alcohol). Sections were prepared for electron microscopy by postfixing in osmium, dehydrating in an alcohol series, and embedding in Durcupan resin (Ted Pella, Redding, CA) and imaging via a CM10 transmission electron microscope (Phillips). 
Therapeutic Caffeination of Researcher - To achieve sufficient levels of cognition to conform to University of Louisville Occupational Health and Safety guidelines, daily consumption of 3-5 aliquots of 8.5g prepared dark blend or similar ground coffee bean (Caza Trail or similar) was performed on a B70 Platinum Brewing System (Keurig) followed by supplementation with $72 \mathrm{mg}$ saccharine (Cumberland Packing Corporation). Physiological changes were monitored and dosing modified as necessary.

Glomerular purification - To obtain 95\% pure glomeruli for RNA extraction, we utilized a common sieving technique in small batches. Briefly, renal cortex is dissected and minced followed by collagenase treatment (Sigma), initial filtration through 100nm nylon mesh (VWR) and glomerular purification and washing with Hanks Buffer (Invitrogen) in a 70nm nylon mesh (VWR). Glomerular samples obtained were determined to be at least $90 \%$ glomeruli by light microscopy with $<5 \%$ tubule contamination.

Purification of Primary Neutrophils from Donor Blood - The Percoll method is a standard density gradient capable of separating cellular components of whole blood. Briefly, whole blood from donors was centrifuged at $500 \mathrm{x}$ g for 35 minutes at $25{ }^{\circ} \mathrm{C}$, with neutrophils forming a distinct band which can be purified. Isolated neutrophils were diluted in endotoxin-free, sterile Krebs buffer. Microscopic evaluation of isolated cells showed that $>92 \%$ of cells were neutrophils. Trypan blue exclusion indicated that $>95 \%$ of cells were viable. The Institutional Review Board of the University of Louisville approved the use of human donors who provided informed consent. 
Stimulation of Primary Neutrophils and Isolation of Granule Contents - Neutrophil granule contents were produced by stimulating donor neutrophils with TNF- $\alpha(2 \mathrm{ng} / \mathrm{mL})$ (Sigma) and $300 \mathrm{nM}$ N-Formyl-Met-Leu-Phe (fMLF) (Sigma) for 30 minutes at $37^{\circ} \mathrm{C}$ in Krebs buffer. Neutrophil cell bodies were removed via centrifugation for 5 minutes at $5000 \times \mathrm{g}$.

Neutrophil Chemotaxis - Donor neutrophils were resuspended at 5 x 104 in Krebs buffer and $100 \mu \mathrm{L}$ of suspended PMNs were allowed to undergo chemotaxis per manufacturer guidelines into $6.6 \mathrm{~mm}$ diameter and $3 \mu \mathrm{M}$ pore size Transwell Permeable Supports (Corning) using a 1:600 dilution of cell culture supernatant in sterile, endotoxin-free Krebs buffer. The Hema 3 protocol (Fisher) was used to fix and stain the membranes.

Neutrophil Degranulation - Degranulation of specific and azurophilic granules was determined by measuring binding of FITC-conjugated monoclonal anti-CD35 (Pharmingen) and FITC-conjugated monoclonal anti-CD66b (Accurate Chemical) on $4 \times$ $10^{6} / \mathrm{ml}$ neutrophils using a FACSCalibur flow cytometer (Becton Dickinson, Franklin Lakes, N.J., USA) as previously described[89].

Nephrotoxic Sera and TAT-SNAP23 Administration - Nephrotoxic sera (a kind gift of Dr. David Salant) or control sera (Rockland) was injected into the tail vein of mice at 1.5mg/25g mouse body weight. TAT-SNAP23 was produced by Dr. Kenneth McLeish. Briefly, human neutrophil SNAP23 was cloned into a pTAT-vector and expressed DH5a 
competent cells (Invitrogen). E. coli BL21-AI cells (Invitrogen) were used to overexpress the recombinant TAT fusion proteins which were purified by Ni-NTA beads (Invitrogen). Administration of TAT-SNAP23 was via tail vein injection at the time of $\alpha$ GBM administration and 6 hours after administration at a concentration of $0.05 \mathrm{mg} / \mathrm{kg}$ body weight. 
CHAPTER III

PROTEOMIC APPLICATIONS FOR DEFINING CELLULAR MECHANISMS AND

DIAGNOSTIC MARKERS OF SYSTEMIC LUPUS ERYTHEMATOSUS

\section{$\underline{3.1 \text { - Overview of mass spectrometry and discovery proteomics }}$}

As described in the Introduction, autoimmune diseases such as systemic lupus erythematosus (SLE) can affect many different body systems, including the central nervous system, blood cells, joints, skin, heart, lungs, and kidneys [11]. The molecular mediators which promote disease pathogenesis are not fully known, thus a primary objective of translational research is the identification of reliable, non-invasive, and quantifiable markers for detection of the early-onset of specific complications. Such markers would enable treatment to be administered most effectively along with assessment of positive response to therapy such that the treatment can be modified or stopped in a timely manner to best manage adverse side effects. These markers also provide important pathogenic insight and tools for testing new or improved therapeutics. In addition to clinical features (i.e. rash, arthritis), diagnostic information for SLE or GN is also provided by measurement of immune cell profiles and activity, identification of 
specific autoantibodies, and identification of changes in protein expression profiles in bodily fluid (i.e. blood, cerebral spinal fluid, lavage fluid, and importantly in GN, urine).

Mass spectrometry-based proteomic technologies have played an important role in each area of clinical diagnosis as well as the development of a more comprehensive understanding of the underlying disease process using a myriad of diverse sample types and techniques. One of the preferred mass spectrometry methods in proteomics combines one or two dimensional (1D or 2D) liquid chromatography (LC) peptide separation with electro-spray ionization (ESI) tandem mass spectrometry (MS/MS) [90]. This LC-MS/MS methodology allows for a direct and highly-sensitive identification of hundreds of individual proteins from virtually any type of biomedical sample [91]. The decision to use 1D versus 2D-LC is based on the complexity of the protein sample. Typically SDS-PAGE bands or spots ( $<30$ proteins) are analyzed by 1D and much more complex samples, such as affinity-purifications and whole cell, tissue, or bodily fluid extracts are analyzed with 2D-LC-MS/MS. Another type of mass spectrometry that is employed is matrix-assisted laser desorption/ionization-time of flight (MALDI-TOF) [92]. With this approach up to 96 individual protein samples are spotted onto a stationary target for analysis. Although the sensitivity of MALDI-TOF is limited to characterizing the 10-15 most abundant proteins in each sample, it has the benefit of being a higher throughput platform for lower complexity, pre-fractionated protein mixtures (i.e. gel separation) because each sample is analyzed in minutes where as a typical 1D-LC run requires an hour and 2D-LC-MS/MS takes 10-12 hours. A third mass spectrometry approach is surface-enhanced laser desorption/ionization-time of flight (SELDI-TOF), a modification of MALDI-TOF [93]. With SELDI-TOF, different surface components, 
such as strong anion exchangers or hydrophobic characteristics, allow binding of proteins with certain characteristics while dissimilar proteins are washed away. This allows analysis of targeted subsets of structurally related proteins and reducing the complexity of the sample improves the overall sensitivity or dynamic range of detection. SELDI-TOF results differ from LC-MS/MS and MALDI-TOF/TOF in that the results are given in mass to charge ratios $(\mathrm{m} / \mathrm{z})$ rather than peptide sequence, so positive protein identification is not possible. It is, however, useful for rapid analysis of the protein $\mathrm{m} / \mathrm{z}$ profiles of semi-complex samples by reducing upfront separation while preserving the fast analysis time of a MALDI platform. Although not as desirable for discovery, these attributes of relative ease of sample preparation and speed of analysis and data output, as well as lower startup and operation costs presents SELDI-TOF as a more suitable mass spectrometry platform for a clinical test.

A key component of these studies is the method used to prepare selectively targeted protein samples for mass spectrometry interpretation. Auto-antigen identification studies usually involve separation of the tissue of interest by $1 \mathrm{D}$ or 2D electrophoresis (1-DE or 2-DE) SDS-PAGE, followed by immuno-blotting with anti-sera from SLE patients, and identification of proteins in immune-reactive bands by MALDITOF/TOF or LC-MS/MS analysis [94]. Another approach isolates autoantibodies from SLE patient serum using immobilized protein G which is then used to affinity enrich antigens in targeted tissue extracts for direct characterization with 2D-LC-MS/MS analysis [95]. As mentioned above, studies comparing diseased and healthy body fluids use two general approaches; 1) 2D gel electrophoresis followed by staining of total protein and MALDI-TOF/TOF identification of the proteins in differing spots or 2) direct 
comparison of total protein expression using LC-MS/MS [94,96]. The work flow of these applications is described in Figure 7. Starting from sample, which could be any preparation of proteins, work flow could lead to a number of separation/purification techniques or proceed directly to tryptic digestion. Both 1-DE and 2-DE are used to immunoblot for novel autoantigens followed by analysis, frequently MALDI or SELDI, but also including 1D and 2D-LC-MS/MS. Complex samples may be separated by affinity chromatography, immunoprecipitation or magnetic bead separation. Techniques may be employed to remove primarily high-abundance proteins, separate based on protein chemistry or interaction kinetics involving immunoglobulins or other proteins. The digested peptides could be subjected to analysis by MALDI, SELDI, and 1D- or 2DESI-LC/MS/MS. Gel separated and digested proteins are frequently analyzed with MALDI or SELDI platforms, but are appropriate for ESI as well. More complex sample types, in particular whole cell lysates, which have not been separated, are frequently subjected to 1D- or 2D-LC peptide separation coupled with ESI-MS/MS analysis. The 2dimentional aspect of LC separation allows a greater number of proteins to be identified from very complex samples when compared to the other methods, with the tradeoff being the greatly increased time (10-12 hours) to analyze one sample. SELDI-TOF and MALDI-TOF are used for profiling while MALDI-TOF-TOF and ESI-MS/MS can sequence the peptides in the sample to positively identify their parent proteins.

Finally, we provide a mechanism by which LC-MS/MS was used to define the proteome of cell culture supernatant which is later assessed for its ability to activate primary neutrophils ex vivo. 


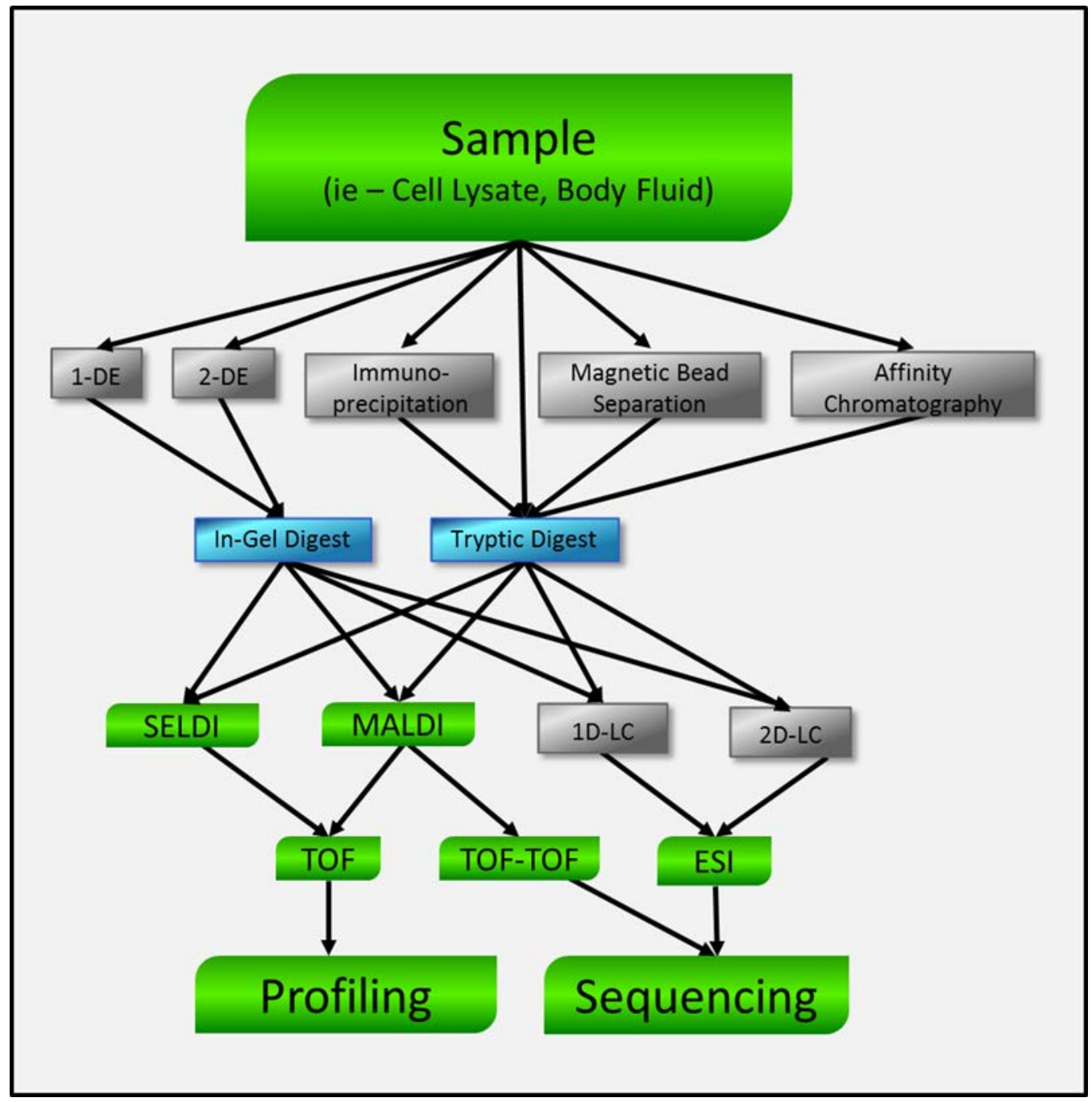

Figure 7 - Flowchart of LC-MS/MS Sample Preparation

Abbreviations: 1-DE, One-Dimensional Electrophoresis; 2-DE, 2-Dimentional Electrophoresis; SELDI, Surface-Enhanced Laser Desorption/Ionization; MALDI, Matrix-assisted laser desorption/ionization; 1D-LC, One-Dimensional Liquid Chromatography; 2D-LC, Two-Dimensional Liquid Chromatography; TOF, Time-ofFlight; ESI, Electrospray Ionization 
Here I seek to explain prior contributions of mass spectrometry techniques to the biological understanding, diagnosis and treatment of SLE, in particular LN, and to lay out a framework for subsequent study of SLE, LN and GN. It is the hope of any investigator that they can catalyze the dissemination of information to allow a greater understanding of complex techniques and illustrate their potential in translational and clinical research related to human diseases. Here I outline relevant contributions of LC-MS/MS and discovery proteomics and adapt protocols which will be utilized to study glomerular disease as described in Chapters IV and V.

\section{$\underline{3.2 \text { - Cardiovascular systemic lupus erythematosus }}$}

Systemic lupus erythematosus (SLE) is an autoimmune condition known to increase a patient's risk for cardiovascular disease (CVD). Even when correcting for potentially confounding factors such as age, genetic background, treatment methodology, family history, disease profile, etc., SLE patients have been shown to have risk up to 50 times above that predicted by Framingham Risk Factors [97-100]. While mortality rates for active disease have fallen in recent decades, mortality rates for CVD in long-term SLE patients continue to rise $[97,100]$. LN occurs in about $50 \%$ of SLE patients and is a major cause of morbidity and mortality, with a significant component attributed to increased risk of ischemic heart disease $[10,101,102]$. The genetic risks, environmental factors, and molecular events that determine susceptibility for LN are unknown. A great deal of effort has been put into understanding the changes to the cardiovascular system in SLE. Cardiac involvement in SLE has been reported with prevalence up to 50\% [103], 
but on average only 9\% will develop true myocarditis [104,105]. SLE has long been known to result in immune deposits in the pericardium and/or myocardium of SLE patients, resulting in both histopathologic and gross anatomic changes [106]. Arrhythmias including sinus tachycardia, atrial fibrillation, long QT, atrioventricular block and bundle branch block are rare complications, but can have significant effects when combined with other factors such as antimalarial therapy [105] and studies have reported up to $16 \%$ of children with SLE to have asymptomatic cardiovascular disease [107]. The risk of developing coronary artery disease is 4-8 times higher in SLE patients than in controls, and this risk increases to 50-fold in young women [98]. Treatment can be tailored to specific patients at risk for the development of such complications, but for this to be possible, early diagnosis of SLE is required followed by the accurate gauging of cardiovascular risk. Mass spectrometry findings have been used to characterize the proteomic changes in the SLE disease state to better understand the pathogenic mechanisms behind cardiovascular disease to allow earlier diagnosis and improved risk assessment [96,108-111]. Relevant results are summarized in Table 1.

To better understand pathogenesis of cardiovascular SLE and provide biomarkers for prompt diagnosis, autoantigens involved in congenital heart block were subjected to proteolytic cleavage with and without sera of SLE patients, followed by MALDI-TOFTOF identification of antibody-protected sites (epitopes) [110]. The resulting peptides were found to correspond to the 200-239 amino acid portion of the Ro52 protein, supporting the use of autoantibodies against Ro52 as a biomarker. Subsequent studies have supported the role of anti-Ro antibodies (both Ro52 and Ro60) in congenital heart 
Table 1 - Contributions of Mass Spectrometry to Cardiovascular Biomarker Discovery in SLE

\begin{tabular}{|c|c|c|c|}
\hline \multicolumn{2}{|c|}{ CARDIOVASCULAR SLE } & \multicolumn{2}{|c|}{ Biomarker or Mechanistic } \\
\hline $\begin{array}{l}\text { Haptoglobin } \\
\text { a2 [112] }\end{array}$ & $\begin{array}{l}\text { MALDI- } \\
\text { TOF-TOF } \\
\text { and ESI- } \\
\text { MS/MS }\end{array}$ & $\begin{array}{l}\text { - Higher plasma Hpa2 seen in SLE vs. } \\
\text { controls } \\
\text { - Supporting research has implicated Hpa2 } \\
\text { isoforms in cardiac complications } \\
{[113,114]}\end{array}$ & Both \\
\hline Ro52 [110] & $\begin{array}{l}\text { MALDI- } \\
\text { TOF-TOF }\end{array}$ & $\begin{array}{l}\text { - Antibody protected sites found with SLE } \\
\text { sera in 200-239 amino acid portion of the } \\
\text { Ro52 protein }\end{array}$ & Both \\
\hline Annexin A6 [96] & LC-MS/MS & $\begin{array}{l}\text { - Autoantibodies identified against Annexin } \\
\text { A6 } \\
\text { - Activation of Annexin A6 via } \\
\text { autoantibody resulting in impaired heart } \\
\text { function }\end{array}$ & Both \\
\hline
\end{tabular}

SLE, Systemic Lupus Erythematosus; MALDI-TOF, Matrix Associated Laser Desorption/Ionization-Time of Flight; ESI, Electrospray Ionization; Ro52 is also known as tripartite motif protein 21 (TRIM21).

Korte et al. 2012. 
block and suggested potential mechanisms for the development of the condition $[115,116]$.

Haptoglobin $\alpha 2$ polypeptide chain differences have been noted between SLE and non-SLE populations via MALDI-TOF-MS [112]. Patients expressing the haptoglobin $\alpha 2$ isoform have been shown to have shorter survival times after coronary artery bypass graft [113] and more severe complications after myocardial infarction [114], possibly due to decreased ability to reduce oxidative stress. The association of haptoglobin $\alpha 2$ peptides with SLE flare may offer insight into the cardiac complications seen in SLE and higher levels of haptoglobin $\alpha 2$ have been reported in SLE patients vs. controls [112].

To identify target autoantigens and explore the pathogenesis of dilated cardiomyopathy, Seko et al. [96] applied a 2-DE separation approach to plasma membrane proteins isolated from rat cardiac myocyte cultures. The protein blot was then probed with serum of healthy controls as well as a 17 year-old patient with an unusual presentation of delayed cardiomyopathy subsequent to neonatal lupus erythematosus. While the control showed no significant reactivity, many immune-reactive spots appeared on the sample probed with SLE serum which were excised and analyzed on a LC-MS/MS system. The analysis identified 2 high-probability proteins: annexin A6, known for calcium signaling and membrane trafficking, and vimentin, known for maintenance of cellular structure (Table 1).

The authors downplayed the likelihood of vimentin playing a role in the pathogenesis of myocardial contractile dysfunction. However, mice with overexpressed Annexin A6 had decreased contraction and relaxation of cardiac myocytes as well as 
dilated cardiomyopathy [117], suggesting that if autoantibodies to annexin A6 stimulate its function, then the result would be suppression of myocardial activity.

\section{$\underline{3.3 \text { - Immune cell involvement in systemic lupus erythematosus }}$}

SLE is, at its core, a disease of the immune system, so a deeper understanding of immune cell function in both normal and diseased states is critical for a comprehensive understanding of SLE. Complementary studies by Dai [118] and Wang [109] show the evolution of a mass spectrometry-based interpretation of peripheral blood mononuclear cells (PBMCs) in SLE. Dai et al. published their work in 2008 in which they compared the relative changes in the proteome of PBMCs of both healthy controls and SLE patients. They employed the conventional approach of 2-DE and silver stain followed by MALDI-TOF/TOF to identify protein spots expressed at different levels. A total of 20 spots differed significantly between the SLE and control group with 11 being upregulated and 9 downregulated. The findings of the study are summarized in Table 2. Briefly, Immunoglobulin $\mathrm{J}$ chain, an early differentiation marker in B cells, was upregulated, as was calprotectin L1, a protein released by granulocytes and monocytes during activation. Interestingly, calprotectin L1 levels were associated with the severity of disease and at the same time were linked significantly to anti-dsDNA antibodies. Glutathione Stransferase was downregulated in SLE, which would reduce the cell's ability to degrade reactive oxygen species, which may contribute to inflammation and tissue damage.

Wang et al. [109] followed Dai two years later with an expanded study which employed the use of isobaric Tagging for Relative and Absolute protein Quantification (iTRAQ) as a means of studying PBMC proteome changes. The iTRAQ method was 
Table 2 - Contributions of Mass Spectrometry to Immunological Biomarker

\section{Discovery in SLE}

\begin{tabular}{|c|c|c|c|}
\hline \multicolumn{3}{|c|}{ IMMUNE CELL INVOLVEMENT IN SLE } & Biomarker or Mechanistic \\
\hline $\begin{array}{l}\text { Immunoglobulin J } \\
\text { [118] }\end{array}$ & $\begin{array}{l}\text { MALDI- } \\
\text { TOF }\end{array}$ & $\begin{array}{l}\text { - Upregulated in SLE } 2.46 \text { fold } \\
\text { - Early differentiation marker for B-cells }\end{array}$ & Both \\
\hline $\begin{array}{l}\text { Calprotectin } \\
\text { L1[118] }\end{array}$ & $\begin{array}{l}\text { MALDI- } \\
\text { TOF }\end{array}$ & $\begin{array}{l}\text { - Upregulated in SLE } 2.02 \text { fold } \\
\text { - Known marker of disease activity in RA } \\
\text { - Released from granulocytes and } \\
\text { monocytes during their activation } \\
\text { - Significantly tied to SLEDAI and higher } \\
\text { levels of anti-dsDNA antibodies and } \\
\text { SLE-arthritis } \\
\text { - May also inhibit Ig production }\end{array}$ & Both \\
\hline $\begin{array}{l}\text { Glutathione S- } \\
\text { Transferase } \\
\text { [109,118] }\end{array}$ & $\begin{array}{l}\text { MALDI- } \\
\text { TOF and } \\
\text { iTRAQ 2D } \\
\text { LC-MS/MS }\end{array}$ & $\begin{array}{l}\text { - Downregulated in SLE } 0.15 \text { fold } \\
(\mathrm{p}=0.001) \text { in one study and vs. diseased } \\
\text { and healthy controls } \\
\text { - Lower GST results in higher ROS levels } \\
\text { and increased oxidative stress }\end{array}$ & Both \\
\hline $\begin{array}{l}\text { Apolipoprotein A- } \\
\text { IV precursor [118] }\end{array}$ & $\begin{array}{l}\text { MALDI- } \\
\text { TOF }\end{array}$ & $\begin{array}{l}\text { - Upregulated in SLE } 2.67 \text { fold }(\mathrm{p}=0.010) \\
\text { - Similar results seen in RA [119] and } \\
\text { Alzheimer’s Disease [120] }\end{array}$ & Biomarker \\
\hline $\begin{array}{l}\text { Zinc finger protein } \\
\text { subfamily } 1 \mathrm{~A}[118]\end{array}$ & $\begin{array}{l}\text { MALDI- } \\
\text { TOF }\end{array}$ & - Upregulated in SLE 2.24 fold $(\mathrm{p}=0.041)$ & Both \\
\hline $\begin{array}{l}\text { Zinc finger protein } \\
\text { - Isoform } 2 \text { protein } \\
549[109]\end{array}$ & $\begin{array}{l}\text { iTRAQ 2D } \\
\text { LC-MS/MS }\end{array}$ & $\begin{array}{l}\text { - Upregulated in active SLE vs. stable SLE } \\
\text { (2.7160), RA (2.3824) and Healthy } \\
\text { Controls (3.1042) }\end{array}$ & Both \\
\hline Resistin [109] & $\begin{array}{l}\text { iTRAQ 2D } \\
\text { LC-MS/MS }\end{array}$ & $\begin{array}{l}\text { - Upregulated in active SLE vs. stable SLE } \\
\text { (3.6784) and Healthy Controls (2.2652) } \\
\text { - Verifies Previous Findings }\end{array}$ & Both \\
\hline S100-P [109] & $\begin{array}{l}\text { iTRAQ 2D } \\
\text { LC-MS/MS }\end{array}$ & $\begin{array}{l}\text { - Upregulated in active SLE vs. stable SLE } \\
\text { (2.9641), and Healthy Controls (2.6475) }\end{array}$ & Both \\
\hline S100-A12 [109] & $\begin{array}{l}\text { iTRAQ 2D } \\
\text { LC-MS/MS }\end{array}$ & $\begin{array}{l}\text { - Upregulated in active SLE vs. stable SLE } \\
\text { (2.3374), RA (2.0595) }\end{array}$ & Both \\
\hline $\begin{array}{l}\text { Brain Acid Soluble } \\
\text { Protein } 1 \text { [109] }\end{array}$ & $\begin{array}{l}\text { ITRAQ 2D } \\
\text { LC-MS/MS }\end{array}$ & $\begin{array}{l}\text { - Upregulated in active SLE vs. stable SLE } \\
\text { (2.1139), Healthy Controls (2.6622) }\end{array}$ & Both \\
\hline $\begin{array}{l}\text { Ras-related C3 } \\
\text { Botulism Toxin } \\
\text { Substrate } 2 \text { [109] } \\
\end{array}$ & $\begin{array}{l}\text { iTRAQ 2D } \\
\text { LC-MS/MS }\end{array}$ & $\begin{array}{l}\text { - Downregulated in stable SLE (0.4663) } \\
\text { vs. diseased controls }\end{array}$ & Both \\
\hline
\end{tabular}

SLE, Systemic Lupus Erythematosus; SLEDAI, SLE Disease Activity Index; Ig,

Immunoglobulin; GST, Glutathione S-Transferase; LN, Lupus Nephritis; LC-MS/MS,

Liquid Chromatography Tandem Mass Spectrometry; MALDI-TOF, Matrix Associated

Laser Desorption/Ionization-Time of Flight; ESI, Electrospray Ionization

Korte et al. 2012. 
used with LC-MS/MS to allow multiplexing of samples (up to 8 per run), which not only saves time, but also reduces technical variability. Using 6 samples each for active SLE, inactive SLE, rheumatoid arthritis (RA) as a diseased control and healthy patient samples, 452 proteins with a variety of cellular locations and biological functions were identified and quantified. Of these, 126 were either upregulated or downregulated across all the groups. When compared to the 2008 data, this study showed a similar downregulation of glutathione S-transferase and upregulation of zinc finger proteins with similar fold changes in both experiments, but also identified several other known SLE regulators and novel functional candidates. In total, 17 proteins were upregulated and 13 were downregulated in active vs. stable SLE. The author's choices for future study are listed in Table 1. Known SLE and inflammatory proteins were identified, such as resistin and calcium-binding family of S100 proteins. Additionally, several novel candidate proteins were identified, such as brain acid soluble protein 1 and ras-related C3 botulism toxin substrate 2, which have roles in apoptotic mechanisms in other diseases [121,122], but this was their first link to SLE disease activity.

\section{4-Lupus nephritis}

Lupus Nephritis (LN) must be considered in a discussion on the use of proteomic methods in lupus, because $\mathrm{LN}$ is one of the most common and most severe complications that can arise from SLE ( 50\%), leading to significant morbidity and mortality [1]. As with other renal disorders, a major diagnostic source for LN is urine. The advantage of using urine as a screening method for presence or severity of disease is the non-invasive 
nature of sample collection. Urine studies also allow long-term observation of patients, allowing tracking of changes in the urine proteome associated with changing disease states. SLE and LN biomarkers have been a topic of discussion dating back to the 1960's and continue to be of interest today [111,123-125]. Recent proteomic studies have provided insight into novel urinary biomarkers for LN and are summarized in Table 3.

Oates et al. [126] built on the ideas of urine protein quantification using 2-DE to train an artificial neural network capable of identifying potentially significant changes to urine proteomes immediately before validation by renal biopsy. Briefly, $200 \mu \mathrm{g}$ protein was collected from 20 different patients with SLE at the time of biopsy. The recovered protein was denatured and separated using 2-DE. A total of 213 protein spots across the 20 patient gels were used to calculate protein abundance and an artificial neural network was trained to identify the class of LN based on the protein abundance profile. The resulting neural network was able to successfully identify $>86 \%$ of all positives and $>92 \%$ of all negatives, with the exception of class $\mathrm{V}$, where each false positive was present in a sample also positive for Class III or IV LN, which were correctly identified. The group then identified the most critical spots for determining sensitivity and subjected each to MALDI-TOF-MS, which identified 6 proteins: $\alpha-1$ acid glycoprotein, $\alpha_{1}$ microglobulin ( 2 spots), zinc $\alpha-2$ glycoprotein ( 2 spots), and IgG $\kappa$ light chain (Table 3 ).

Early work successfully identified proteomic profiles of renal flare in SLE through the use of SELDI-TOF to identify protein signatures without the need to positively identify proteins. This allowed the identification of novel biomarkers, but the protein identification limitation of the SELDI-TOF technique prevented mechanistic inference and predictions [127]. Zhang et al. [128] provided both diagnostic and 
mechanistic understanding of LN using longitudinal urine proteomics to assess the changes in the urine proteome before, during and after SLE renal flare by combining SLEDI-TOF with LC-MS/MS sequencing for protein identification. Using a sample set of 145 urine specimens from SLE Class III, IV and V patients, they identified 27 low molecular weight proteins that showed significantly different levels of expression before, during or after SLE flare. While no single peptide was present in all the flare or non-flare samples, combinations of two peptides were found that successfully differentiated $100 \%$ of the remission and pre-flare samples from flare and post-flare samples. LC-MS/MS analysis identified multiple isoforms of $\alpha-1$ Antitrypsin, albumin, and hepcidin as proteins of interest (Table 3). Interestingly, Hepcidin-20 levels were only altered preflare, which means Hepcidin-20 may be a useful indicator of impending flare, allowing modulation of clinical management to reduce severity and duration of the flare. A follow up study two years later supported this idea and further presented IL-6 and INF $\alpha$ dependent upregulation of Hepcidin transcription as a possible mechanism of Hepcidin increase in LN [129].

A study using LC-MS/MS to characterize immune-reactive bands from 2-DE separated endothelial cell lysate with sera of SLE patients, other autoimmune conditions and healthy controls identified the glycolytic enzyme aldolase A as a candidate target antigen [111] (Table 3). Moreover, this was found to be more common for LN patients than in SLE without LN (43.4\% with LN vs. 11.1\% without LN). However, reactivity to aldolase A was not appropriately significant between LN and other inflammatory disease controls such as RA and polymyositis to be used as a stand-alone biomarker for LN. The 
authors did however note specificity of LN patient anti-sera to the 94-183 aa region of aldolase A over other autoimmune conditions.

Suzuki et al. used a combination of SELDI-TOF [130] and MALDI-TOF [131] for evaluation of the urine proteome in pediatric LN (Table 4). Four potentially significant biomarkers were identified including transferrin (Tf), ceruloplasmin (Cp), $\alpha 1$ acid-glycoprotein (AGP), and lipocalin-type prostaglandin-D synthetase (L-PGDS), each with great significance $(\mathrm{p}<0.005$, Table 3$)$. Significant increases of Tf, L-PGDS, and AGP were seen as early as 3 months before clinical diagnosis or worsening of LN. Both Tf and AGP were also seen in adult forms of the disease [132], while L-PGDS is novel to pediatric LN diagnosis. Cp has been implicated in global SLE, but was not found to be an effective metric for defining the course of $\mathrm{LN}$ in pediatric patients.

\section{$\underline{3.5 \text { - Neuropsychiatric systemic lupus erythematosus }}$}

Neuropsychiatric SLE (NPSLE) is a potentially severe complication that varies in its prevalence between 9.5\%-95\% based on the literature cited and the diagnostic criteria [133]. Findings suggest that NPSLE can be present in a mild form in as many as $95 \%$ of SLE patients [134], making it more common but typically less severe than renal or cardiovascular complications. Mood disorders and cognitive disorders are common symptoms and can be particularly challenging to diagnose in pediatric and adolescent patients due to the rapid emotional and physical changes that occur at those ages. While acquisition of cerebral spinal fluid (CSF) is invasive, it has become routine in the 
Table 4 - Contributions of Mass Spectrometry to Biomarker Discovery within Pediatric Populations of Lupus Nephritis Patients

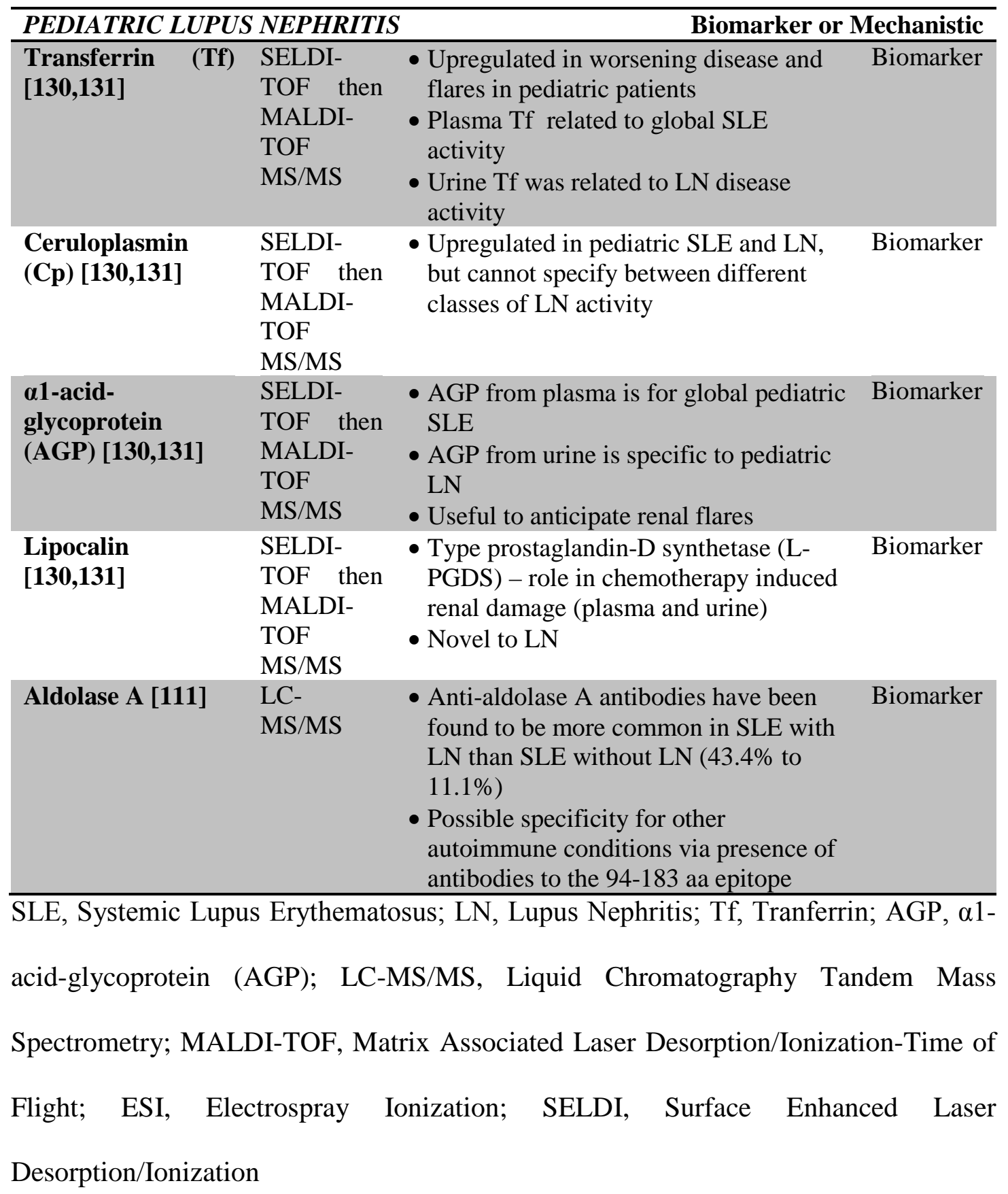

Korte et al. 2012. 
diagnosis of many neurological diseases and therefore in addition to serum, CSF is a useful medium for proteomic study of NPSLE. NPSLE research has focused on both biomarker identification for its diagnostic value as well as autoantigen identification for improved mechanistic understanding of the disease state and potential therapeutic development (Table 5).

Lu et al. [94] found CSF and sera obtained from NPSLE patients to be reactive to rat, mouse and monkey brain homogenate when separated by 2-DE. MALDI-TOF-TOF was used to identify immune-reactive proteins. A total of 275 serum samples and 177 CSF samples were included, giving a great deal of depth and statistical power to the study. Briefly, four anti-ANA-positive serum samples, which did not react to Hep2 cells, were used as pilot samples. Initial analysis of the reactive spots revealed an interaction in 3 of 4 samples with $\alpha$-internexin (INA), a highly conserved protein between rodents and humans (Table 5). Recombinant human INA was then used in a western blot to confirm reactivity of sera as well as immunofluorescence with sera and anti-INA polyclonal antibody (pAb). Both the sera and the pAb were found to react to cultured rat cortical neurons and the reactivity of sera was reduced by incubation with recombinant INA. These findings opened the door for analysis of the entire cohort of CSF and serum samples. Using 2 standard deviations as a cutoff for positivity, $41.7 \%$ of the NPSLE samples of CSF were found to be positive for anti-INA antibodies (as compared to $0 \%$ of SLE with cerebral infarction, $7.1 \%$ of normal SLE controls, and $10 \%$ of other neurological diseases). In total, the data showed reactivity in $>40 \%$ of NPSLE patients in a dose dependent manner toward INA. Longitudinal studies found that antibody levels were inversely proportional to cognitive level and declined after initiation of therapy and 
Table 5 - Contributions of Mass Spectrometry to Biomarker Discovery in

\section{Neuropsychiatric SLE}

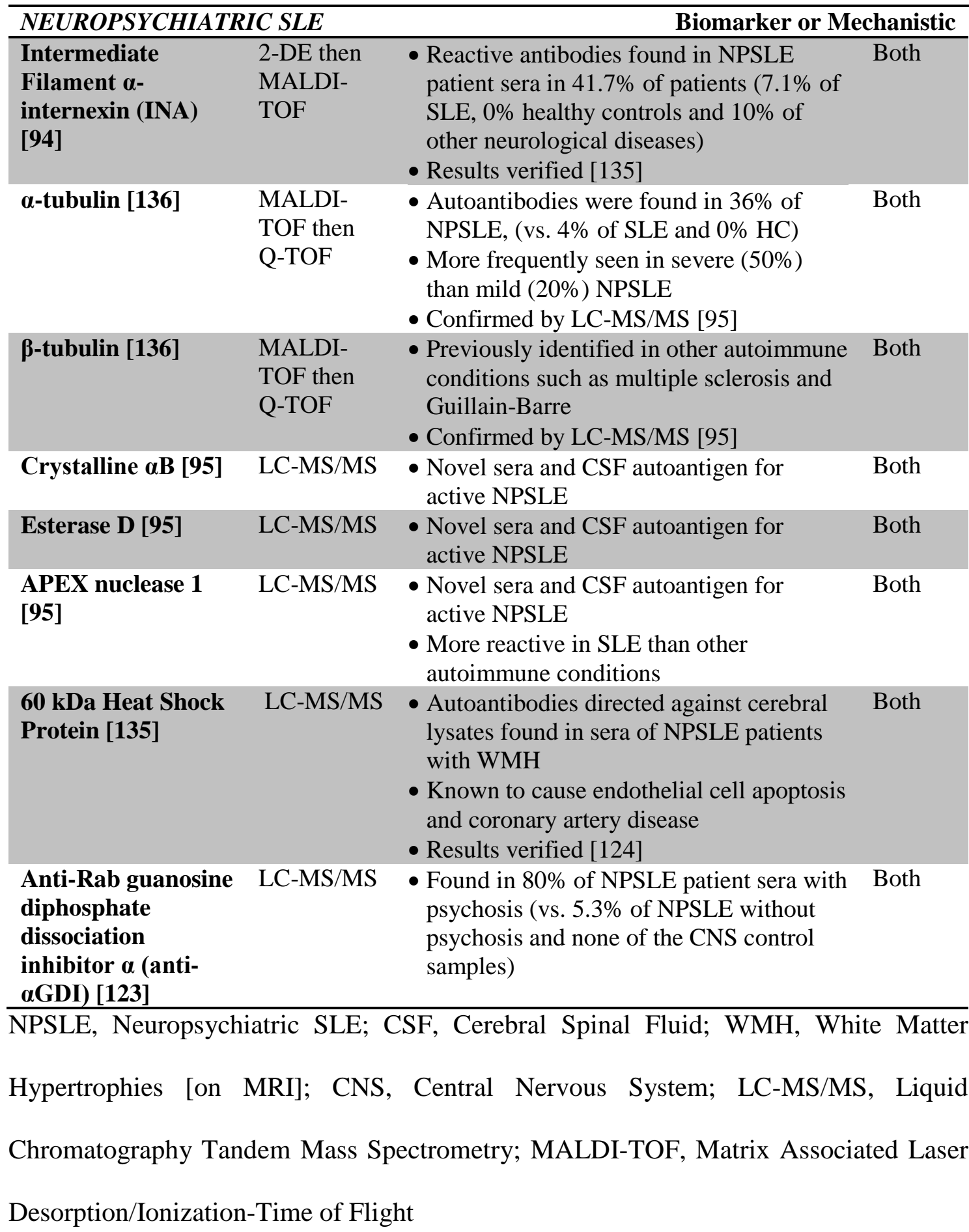

Korte et al. 2012. 
clinical remission. Furthermore, mice immunized with INA plus LPS developed cognitive impairments similar to NPSLE and exhibited hippocampal and cortical neuron apoptosis, suggesting that the presence of anti-INA antibodies in the serum of patients was pathogenic.

Ndhlovu et al. [136] identified $\alpha$ - and $\beta$-tubulin as target autoantigens for NPSLE using patient sera to probe isolated mitochondria from bovine brain followed by MALDITOF analysis. Autoantibodies to $\beta$-tubulin have been identified in other CNS targeting autoimmune diseases such as Guillain-Barre syndrome, Multiple Sclerosis, and Sydenham's chorea, but targeting of $\alpha$-tubulin is potentially specific to NPSLE. $\alpha$ tubulin autoantibodies were present in $36 \%$ of NPSLE patient sera, only $4 \%$ of SLE patients without neurological symptoms and none of the MS, epileptic or healthy control samples (Table 5). Possibly due to the small size of the NPSLE population in the study $(n=37)$, no correlation was seen between specific symptoms of NPSLE and the presence of $\alpha$-tubulin autoantibodies in sera. Larger patient population studies are needed to further explore the involvement of anti- $\alpha$-tubulin antibodies in NPSLE.

LC-MS/MS incorporates a direct front-end separation of complex samples to greatly increase the sensitivity of the procedure for low abundance protein detection. LCMS/MS may be superior to MALDI-based techniques when dealing with complex samples. Katsumata et al. found this to be true while attempting to uncover novel serum autoantibodies for SLE, in particular those involved in CNS syndromes [95]. Whole protein extracts from human neuroblastoma and glioblastoma cell lines were separated using 2-DE and reacted to patient sera. It was determined that MALDI-TOF sensitivity was too low for effective protein identification in this case. To address this concern, anti- 
sera from SLE patients with and without CNS syndromes was bound to Protein GSepharose beads and incubated with the same cell line extracts. Proteins pulled down with the Sepharose beads were analyzed with a LC-MS/MS method using a Q-TOF instrument. Most of the 154 proteins identified by the LC-MS/MS approach were not identified in the 2-DE/MALDI-TOF analysis and 50 were found in the CNS group but not the non-CNS group. After exclusion of previously described autoantigens, 11 recombinant candidates showed reactivity against pooled serum of 28 patients with active CNS SLE by western blot analysis (5 of which were only detected by LC-MS/MS). These 11 candidates were then probed with sera of 106 SLE patients (42 with active CNS syndromes) as well as 100 healthy controls, resulting in a list of 5 confirmed autoantigens, 3 of which were novel (crystalline $\alpha \mathrm{B}$, esterase D and APEX nuclease 1, Table 1). When compared to other diseased sera (RA, SSc, SS, and MS), levels of anticrystalline $\alpha \mathrm{B}$ was not found to be significantly different. Anti-APEX nuclease 1 and esterase D, on the other hand, had significantly higher levels in SLE than in these disease controls and between CNS and non-CNS SLE sera. APEX nuclease 1 is the major apurinic/apyrimidinic endonuclease in human cells and is found to be upregulated in tissues under oxidative stress and has a significant effect on apoptotic regulation in certain tissues [137]. The effects of esterase D are less well known.

In a similar study, Kimura et al. used a combination 2-DE and LC-MS/MS with an ESI-LCQ instrument to obtain higher sensitivity for low abundance proteins [135]. Patient sera from a NPSLE patient with white matter hyperintensities (WMH) on T2weighted MRI scan was reacted to a 2-DE rat cerebral lysate blot. In total, 9 reactive spots were seen and 5 were analyzed by LC-MS/MS. The 2 spots of greatest intensity 
corresponded to $60 \mathrm{kDa}$ Heat Shock Protein (Hsp60) [135], which functions to refold proteins that are misfolded and/or facilitate degradation of proteins that cannot be recovered [138]. Enhanced expression of Hsp60 can induce endothelial cell apoptosis and Hsp60 expression on the surface of endothelial cells have been reported to be directly related to coronary artery disease [139], opening the possibility of a similar relationship in the cerebral vasculature. In a follow-up study of 180 patients with neurological diseases (including 15 with SLE) and 23 healthy controls, Kimura et al. showed that the titer levels of anti-Hsp60 were directly correlated to severity of $\mathrm{WMH}$ in the brain, suggesting the presence of anti-Hsp60 as both a diagnostic tool as well as a mechanistic agent in the development of NPSLE [124]. Although the authors chose to focus on Hsp60, the study also found SLE specific antibodies to $\alpha$-internexin, $\beta$-actin, and glial fibrillary acidic protein (Table 5).

Another study using LC-MS/MS also suggested a relationship between NPSLE exhibiting psychosis with anti-Rab GDI- $\alpha$ [123]. LC-MS/MS and WB analysis of sera found that 4 of 5 patients with NPSLE with psychosis (80\%) expressed anti-Rab GDI- $\alpha$ (Table 5), while 1 of 13 patients with NPSLE and none with psychosis (5.3\%) expressed the autoantibody, along with no expression in patients with multiple sclerosis $(n=12)$, infectious meningoencephalitis $(n=13)$, polyneuropathy $(n=10)$, psychotic syndromes $(n=10)$, or healthy controls $(n=12)$.

\section{6 - Other uses for proteomics in the study of SLE}


Aside from assessing differences in disease states, pharmacologic reactions can be measured using proteomic techniques. Kao et al. [140] used a 2-DE and MALDI-TOF to investigate the changes to apoptotic-prone macrophages (APM) from lupus-prone NZB/W-F1 mice before and after treatment with cystamine, a known inhibitor of transglutaminase 2 (TG2) activities. A number of the findings indicated that cystamine has anti-apoptotic affect in the APMs and suggests possible mechanism of this activity. Protein expression and enzyme activity of Caspase 3 were downregulated and antiapoptotic proteins APIP, PEBP4, and Bcl-2 were upregulated. ERK 1/2 showed reduced phosphorylation after treatment in APMs, which also would result in survival of APMs and fewer apoptotic bodies to serve as potential targets for autoantibody production. Previous studies have shown that ERK 1/2 phosphorylation is increased with cystamine treatment in endothelial cells [141] and neuronal tissue [142], therefore the findings of decreased phosphorylation in APM may help further explain the development of SLE in specific tissues and lead to a more complete model of pathogenesis.

Mass spectrometry can also be useful as a supplemental technique of a study. In work primarily focused on genetics, D’souza et al. [143] utilized MALDI-TOF-MS to support the hypothesis that catalase polymorphisms affect the risk and severity of SLE. Oxidative processes resulted in the 4-hydroxy 2-nonenol (4-HNE) adducts on membrane catalase of red blood cells (RBCs). MALDI-TOF was of great use in this situation because it allowed the positive identification of catalase from RBC membranes (an uncommon location) while migrating at a higher molecular weight, possibly due to 4HNE adducts. Ye et al. [144] expanded on previous microarray data by selecting a gene that is upregulated in SLE (interferon-induced with tetratricopeptide repeats 1 - IFIT1), 
creating a IFIT1 fusion protein with GST, and then using bead immobilized GST-IFIT1 as bait to capture interacting proteins in white blood cell lysate. MALDI-TOF was performed on the proteins pulled down in the procedure and found Rho/Rac guanine nucleotide exchange factor to be a potential target for IFIT1 interaction in SLE. Follow up studies have supported the role of the interferon pathway in SLE and other autoimmune diseases [145-147].

\section{7 - Characterization of the Secretome of Cultured Cells}

Cell culture provides an opportunity to isolate a cell type from the organism to analyze its function in a controlled setting. It is not possible to replicate all biological events which would take place in a mammalian system, but experimental models utilizing cell culture for initial discovery can be useful to identify low abundant targets or factors from specific cell populations which would otherwise be masked in a typical body fluid sample.

The global hypothesis of this dissertation succinctly states that podocyte activity can promote the development of GN, and that ABIN1 is important to prevent inflammation in local tissues. This is not, however, to say that cells of the immune

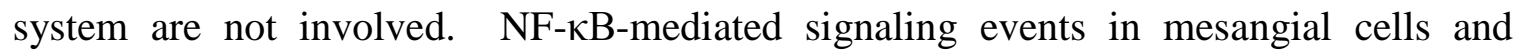
podocytes are known to promote synthesis and secretion of pro-inflammatory cytokines and chemokines which recruit neutrophils, macrophages and other immune cells to tissue[148-152]. Normal immune cell functions in these tissues include clearing of infectious material and cellular debris, while inappropriate or excessive recruitment by 
podocytes and mesangial cells to otherwise healthy tissue can lead to inflammatory damage. The renal secretome is an incompletely characterized collection of factors released by the cells of the kidney[152]. We characterized the normal secretome of podocytes and simultaneously investigated ABIN1-related changes to the secretome which may attract excessive numbers of immune cells to the kidney, resulting in local conditions which may promote LN [148-151,153-158]. This was accomplished through a relatively unbiased analysis of the secretome of each cell type under varying conditions with two-dimensional liquid-chromatography tandem mass spectrometry (2D-LCMS/MS).

Method development focused on establishing a sample of appropriate purity but with sufficient chemical properties suitable for LC-MS/MS analysis. Three factors were of great importance: (1) the chosen stimulant, TNF- $\alpha$, could mask low abundant cytokines via ion suppression and additionally would hinder measurement of TNF- $\alpha$ produced by the podocyte, (2) the proteomic makeup of the cell culture media would have to be sufficient for LC-MS/MS analysis, and (3) the media had to be sufficient for proper podocyte cell functions and could not induce unnecessary stress on the cells which would undoubtedly affect their inflammatory profile. Podocyte cell culture media is comprised of $10 \%$ FBS, which contains a host of protein “contaminants", and phenol red can confound LC-MS/MS analysis and should be avoided. In addition to the chemical components of the media, the timing of stimulation and sample collection need to be optimized for each cell type used and for each stimulant. Following a number of preliminary experiments, I arrived at a protocol which accomplished all of these preconditions. Podocytes were stimulated in $10 \mathrm{~cm}$ dishes for 6 hours in $10 \mathrm{~mL}$ serum- 
free media, followed by 3 washes in 10mL warm Krebs buffer, and a 6 hour collection in warmed Krebs buffer. Figure 8 outlines the kinetic properties of cultured podocytes stimulated from 0-18 hours with TNF- $\alpha$ and shows that a 6 hour stimulation was sufficient to induce production of 3 important proinflammatory cytokines, MCP-1, GMCSF, and IL-8. In addition, I also was able to remove the TNF- $\alpha$ used for stimulation to below detectible levels of our ELISA assay $(<<2 \mathrm{pg} / \mathrm{mL})$. This was important for both LC-MS/MS analysis as well as downstream characterization of these samples using biological methods (see Chapter V).

Once optimal timing and media components were achieved, sample analysis had to be addressed. Most cytokines are small proteins and we have found that sample purity is increased through a 10 minute centrifugation in an Amicon Ultra-30kDa Centrifugal Filter Unit (Millipore, Billerica, MA) to remove proteins larger than $30 \mathrm{kDa}$, followed by standard trypsin digest and LC-MS/MS sample preparation as described in the materials and methods. Figure 9 shows protein identifications without this cutoff and includes a number or large, high abundant proteins such as fibronectin and actin. Figure 10 outlines proteins identified after the $30 \mathrm{kDa}$ cutoff was applied before trypsin digestion. A number of cytokines expected to play a role in GN are shown to be upregulated in the podocyte secretome, including IL-6, MCP-1 (CCL2) and the cell adhesion molecules VCAM1 and ICAM1. The benefit of mass spectrometry-based approaches is the ability to identify novel proteins from a given sample, and a number of promising candidates for future study were identified such as transgelin and TIMP1 which have a role in matrix remodeling and perlecan, filamin, cofilin and moesin. Of note, a side project involving 

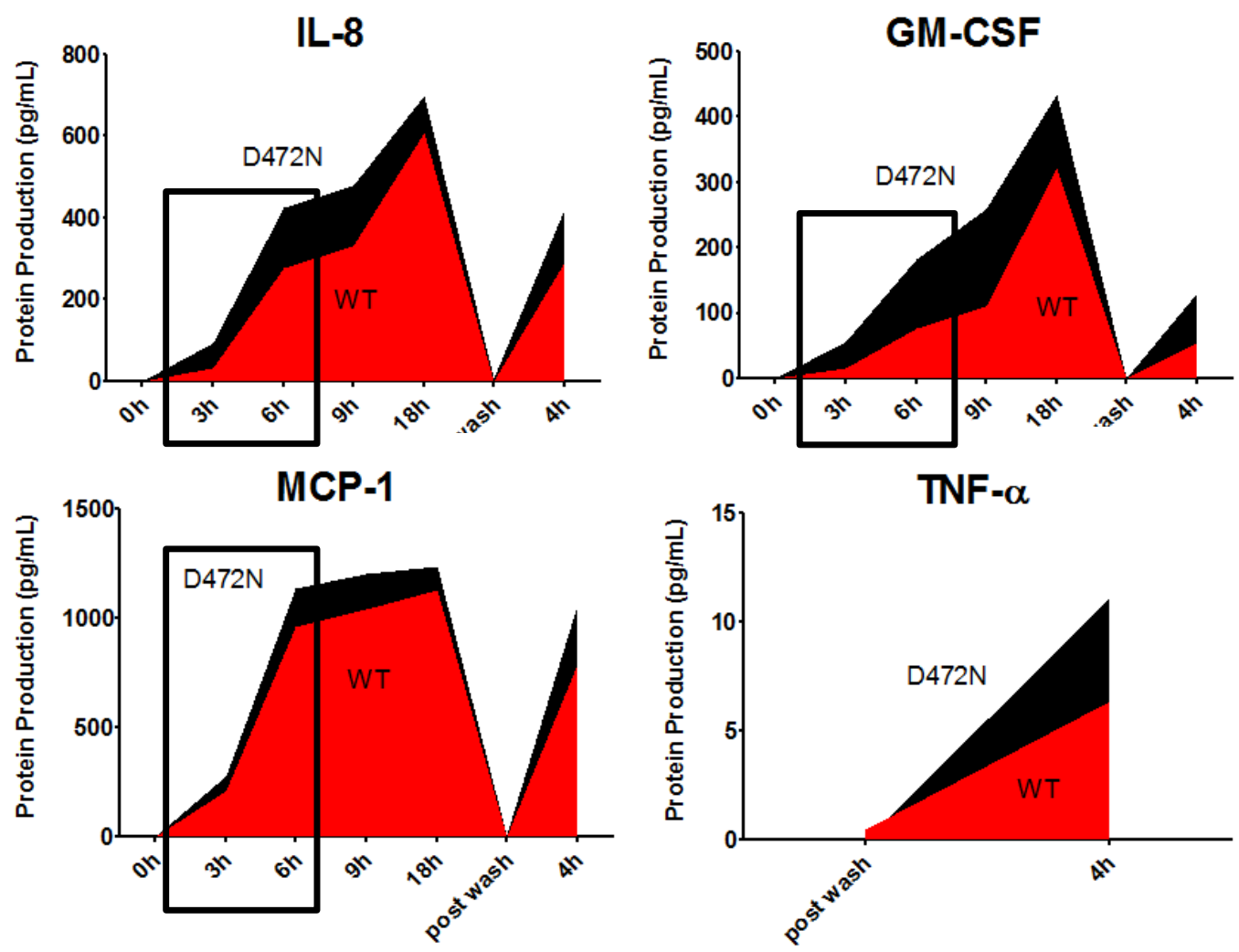

Figure 8 - Kinetics of Cytokine Secretion by Podocytes in Response to TNF-a

\section{Stimulation}

Podocytes were produced and treated as described in the Materials and Methods.

TNF- $\alpha$ stimulation $(100 \mathrm{ng} / \mathrm{mL})$ at time points from $0-18$ hours was used to determine the kinetics of cytokine production using ELISA to measure cytokine in $\mathrm{pg} / \mathrm{mL}$. It was found that from $0-6$ hours of TNF- $\alpha$ stimulation, TNF- $\alpha$ response resulted in high levels of cytokine production. Further, the wash step following the 18 hour time point resulted in all analytes dropping below their detectible level for their respective assays, and this included TNF- $\alpha$ which was the stimulant. 


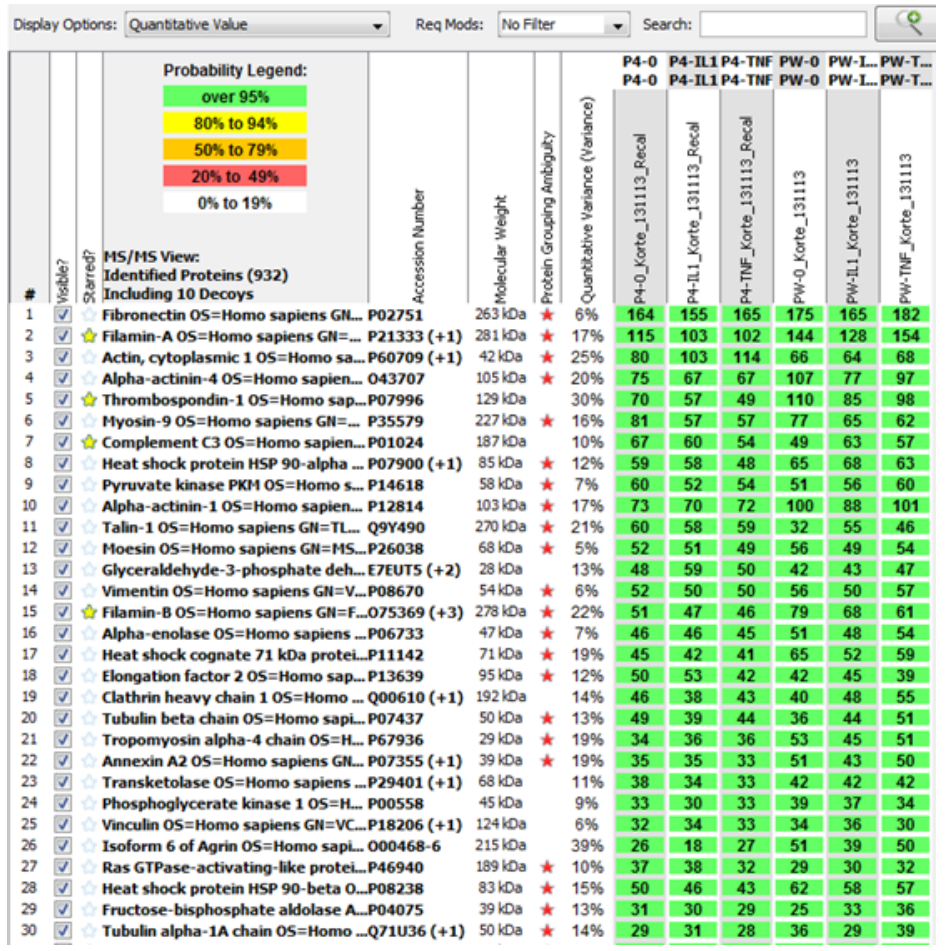

Figure 9 - Identification of Total Protein Secreted from Cultured Podocytes

Cell culture supernatant was prepared from cultured podocytes as described, digested and prepared for LC-MS/MS analysis. Identified peptides were predominantly highly abundant matrix proteins and large cytosolic proteins, rather than the typically small $(<30 \mathrm{kDa})$ cytokines and chemokines. This analysis identified 932 proteins and 35,075 unique spectra.

Erik Korte. Unpublished. 


\begin{tabular}{l|r|r|r|r|} 
& \multicolumn{2}{c}{ Unstim } & \multicolumn{2}{r}{ TNF- $\alpha$ Stimulated } \\
Symbol & \multicolumn{1}{|c}{ WT } & \multicolumn{1}{c}{ D472N } & \multicolumn{1}{c}{ WT } & \multicolumn{1}{c}{ D472N } \\
\hline VIM & 120.960 & 65.491 & 101.780 & 68.065 \\
\hline IL6 & 5.125 & 6.742 & 4.425 & 10.256 \\
TAGLN & 5.125 & 12.520 & 6.638 & 16.783 \\
HSPG2 & 18.451 & 52.008 & 17.701 & 61.538 \\
SCRN1 & 1.025 & 2.889 & 1.106 & 4.662 \\
GREM1 & 3.075 & 9.631 & 4.425 & 8.392 \\
LGALS3BP & 1.025 & 10.594 & 1.106 & 8.392 \\
SERPINH1 & 5.125 & 9.631 & 5.532 & 9.324 \\
CNN3 & 6.150 & 10.594 & 4.425 & 10.256 \\
LUM & 5.125 & 9.631 & 4.425 & 10.256 \\
ANXA3 & 4.100 & 7.705 & 2.213 & 10.256 \\
\hline TMSB4X & 13.326 & 22.151 & 17.701 & 11.189 \\
SERPINA1 & 18.451 & 29.856 & 21.020 & 38.228 \\
MSN & 29.726 & 41.414 & 42.040 & 50.350 \\
STIP1 & 13.326 & 10.594 & 12.169 & 7.459 \\
FLNC & 12.301 & 9.631 & 8.850 & 7.459 \\
CFL1 & 37.927 & 15.410 & 29.870 & 13.986 \\
TPT1 & 2.050 & 6.742 & 4.425 & 4.662 \\
CCL2 & 4.100 & 3.852 & 5.532 & 4.662 \\
COL4A1 & 1.025 & 8.668 & 1.106 & 4.662 \\
\hline ILF3 & 4.100 & 5.779 & 6.638 & 5.594 \\
BMP1 & 1.025 & 4.816 & 1.106 & 5.594 \\
C1S & 2.050 & 5.779 & 1.106 & 5.594 \\
NPC2 & 3.075 & 2.889 & 2.213 & 5.594 \\
VCAM1 & 0.000 & 0.000 & 1.106 & 5.594 \\
TIMP1 & 5.125 & 7.705 & 5.532 & 7.459 \\
\hline
\end{tabular}

Figure 10 - Identification of Proteins Secreted by Podocytes Following 30 kDa Size-Selective Filtration

Most cytokines and chemokines are small peptides of less than $30 \mathrm{kDa}$. Thus, to prevent contamination and ion suppression from highly abundant proteins, an Amicon Ultra-30kDa Centrifugal Filter Unit from Millipore was used to remove proteins larger than $30 \mathrm{kDa}$ before tryptic digestion. The heatmap represents the Protein Abundance Factor (PAF) which provides a quantitative value for each identified peptide while taking into account the total protein's size, with the understanding that larger proteins will produce more digested peptide fragments and thus will artificially result in higher numbers of peptide identification. 
moesin has suggested it may be directly involved in the pathogenesis of GN as an autoantigen for a small population of patients with membranous glomerulonephropathy.

\section{$\underline{3.8-\text { Conclusions }}$}

Many of the studies presented in this review were aimed at using proteomic applications to define events in specific SLE complications, but it is our view some of these discoveries could also provide insight into other areas of SLE or that approaches applied to similar diseases could be modified for use in SLE. Recent studies have shown the use of MALDI-TOF techniques for synovial fluid analysis in autoimmune arthritis [159], which could be used to assess changes in SLE arthritis. Kimura et al. [135] identified a $60 \mathrm{kDa}$ Heat Shock Protein as an autoantigen in NPSLE, a protein with known ties to coronary artery disease and endothelial cell apoptosis [139,160,161] which may play a role in the development of cardiovascular SLE. Several studies have shown the benefits of proteomics using bronchoalveolar lavage fluid (BALF) as a sample for the study of sarcoidosis [162], systemic sclerosis [163,164], and other pulmonary diseases $[165,166]$. The use of BALF for the study and diagnosis of pulmonary complications in SLE would be less invasive and safer than biopsy. Tamburro et al. [167] recently published a proteomic review of the vitreous humor of the eye. Contrasting these findings to the proteome of SLE patient's vitreous humor could shed more light on the pathogenesis of retinopathy in SLE. Dai et al. [118] found Immunoglobulin $\mathrm{J}$ to be upregulated in peripheral blood mononuclear cells. Other mass spectrometry-based techniques could be 
used such as multiple reaction monitoring (MRM) and Selected Reaction Monitoring (SRM) LC-MS/MS, which allows focused study on only $\mathrm{m} / \mathrm{z}$ ratios of interest, reducing sample processing time and greatly increasing throughput. After the initial biomarker discovery stages, MRM/SRM has been used as a tool in biomarker validation in blood [168] and synovial fluid, specifically in RA patients [169]. Additionally, the speed and potential for multiplexing means MRM/SRM could have clinical utility in patient diagnosis, prognosis, and monitoring [170]. We used a LC-MS/MS approach to identify the $\mathrm{M}$-type phospholipase $\mathrm{A}_{2}$ receptor $\left(\mathrm{PLA}_{2} \mathrm{R}\right)$ as the target antigen in idiopathic membranous nephropathy (iMN) [5]. Antibodies recognizing the $\mathrm{PLA}_{2} \mathrm{R}$ were present in $70 \%$ to $80 \%$ of iMN patients. However, they are not present in any patients with membranous lupus nephritis (MLN), which develops in up to $40 \%$ of patients with LN [171]. This suggests that there are unique autoantibodies directed against specific kidney proteins other than the PLA $2 \mathrm{R}$ in MLN. Therefore, proteomic studies could be designed to identify such target antigens as well as specific epitopes important in MLN.

Even with the strides we have made in the understanding of SLE, it is still a debilitating condition effecting hundreds of thousands of patients every year. While treatments are available, they bring with them significant side effects and frequent failure rates. Recent advances in mass spectrometry technology have allowed an even more thorough analysis of complex samples to better understand the significance of minute changes in the pathogenesis of SLE, while accommodating large sample sizes through multiplexing and high throughput methods. The method I outline here can serve as a starting point for optimization of LC-MS/MS analysis for the secretomes of many diverse cell populations and may have applications in primary body fluid as well. The options 
researchers or clinicians have in mass spectrometry techniques are rapidly expanding and have the ability to be tailored to a specific study. It is my hope that the characterization of the potential applications of mass spectrometry will provide a greater knowledge of the diverse uses of mass spectrometry and other proteomic technologies in the study of SLE, GN and other conditions. While a proteomic understanding of disease is by no means a panacea, it offers the potential to help advance our knowledge and understanding of SLE to a point that the disease could be less of a burden for those afflicted. 
CHAPTER IV

ABIN1 DYSFUNCTION AS A GENETIC BASIS FOR LUPUS NEPHRITIS

\section{$\underline{4.1-\text { Introduction }}$}

As stated previously, SLE is a multi-system autoimmune disease that is characterized by an abnormal immune response leading to autoantibody production, immune complex formation, $\mathrm{T}$ cell activation, and inflammatory cytokine release [8]. Multiple factors contribute to SLE and LN is a frequently associated condition which is devastating to the patient $[8,10]$. The pathogenesis of $\mathrm{LN}$ is variable among different ethnic groups showing a genetic link, but current therapies are ineffective in half the population and carry major side effects $[4,14]$.

Our lab has previously characterized SLE-like autoimmunity in a mouse model expressing ABIN1[D485N] [53]. Due to the more than casual relationship between SLE and LN, I asked if these mice could present a renal phenotype which would shed light on the major glomerular events in the pathogenesis of LN. A major symptom of renal dysfunction is protein loss into the urine from resulting from glomerular damage [16]. Urine albumin:creatinine ratios (UAC) present a method for analysis of spot urine by 
measure the serum protein albumin against the level of creatinine in the urine which is freely filtered into the urine and not secreted to a great degree by tubule cells, and thus provides an appropriate normalization factor for glomerular filtration compared to reabsorption of water in the tubules [172].

Physiological changes in both mouse models and human disease include foot process effacement and expansion of the mesangial matrix and will be described in this spontaneous model of autoimmunity to further suggest a GN-like phenotype [17]. Complement component 3 (C3) plays a central role in the innate immune response and inflammation, and low serum C3 is a diagnostic criteria for SLE [173]. Here I seek to characterize the changes in this model of spontaneous autoimmunity as it pertains to human disease, and begin to identify the molecular mechanisms responsible for the pathogenesis of LN with the hope to define more specific diagnostic and therapeutic targets.

\section{$\underline{4.2-\text { Results }}$}

\section{Renal Pathophysiology of ABIN1[D485N] knock-in mice}

We reported previously that ABIN1[D485N] mice develop antibody-mediated glomerulonephritis [53]. The aim of this study was to provide a complete characterization of the renal abnormalities in these mice. Table 6 presents the evaluation of the histologic grading of glomerular changes in wild-type (WT) and ABIN1[D485N] mice at 3-4 months and 5-6 months of age. The scoring system used for histologic grading is 


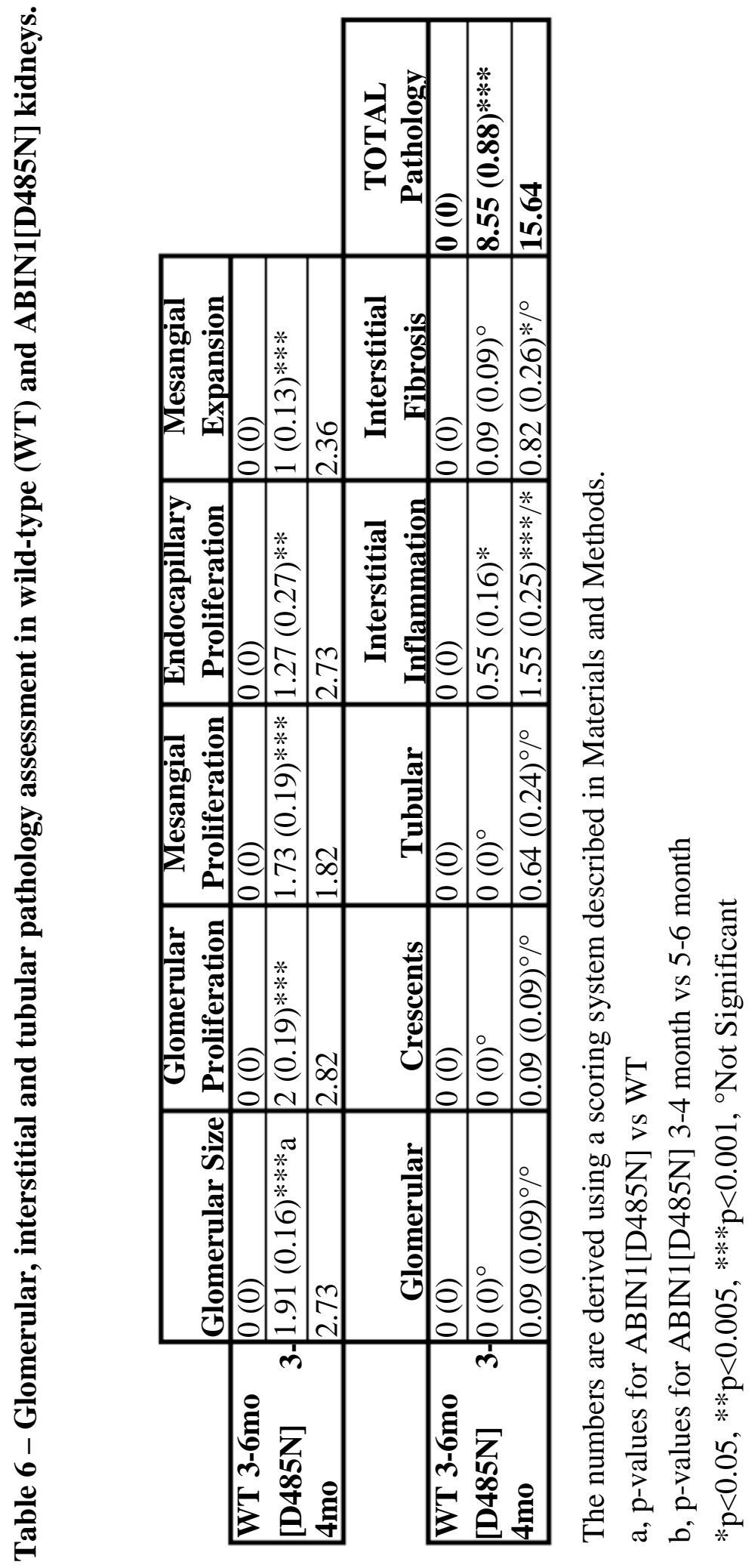


described in Chapter 2. Glomerular size, mesangial and endocapillary proliferation, and mesangial expansion in ABIN1[D485N] mice were all significantly increased by 3-4 months. By 5-6 months ABIN1[D485N] mice demonstrated a significantly progressive increase in glomerular size, endocapillary proliferation, and mesangial expansion, when compared to 3-4 month old ABIN[D485N] mice (Figure 11a). All mice at 3-4 months of age demonstrated glomerular hypercellularity, with 50\% showing severe (3+) changes. Hypercellularity was focal in 2 mice and diffuse in 9. At 5-6 months all mice showed diffuse hypercellularity and all but one were histologically graded as severe $(3+)$. An increase in mesangial matrix was seen in 8 of 11 mice at 3-4 months, but was only mildly increased in 3 of those 8 mice. At 5-6 months, all but one mouse demonstrated a moderate to severe increase in mesangial matrix. Crescents were only observed in 2 mice at 5-6 month, and glomerular sclerosis was seen in 4 of those mice.

Table 6 and Figure 11b show that there was a significant amount of interstitial inflammation in ABIN1[D485N] mice at 3-4 months of age, while little or no tubular atrophy or interstitial fibrosis was observed. By 5-6 months, there was a significant increase in interstitial inflammatory cell infiltration, tubular atrophy and interstitial fibrosis (Figure 11b). Mild interstitial inflammation was seen in only 4 of 11 mice at 3-4 months. By 5-6 months, all mice had some degree of interstitial inflammation, which was moderate or severe in 8 of 11 mice. Mild, patchy tubular atrophy was seen in less than $50 \%$ of mice in each age group. A sparse amount of tubulointerstitial fibrosis (TIF) was seen in 2 of 11 mice at 3-4 months and mild to moderate TIF was observed in 6 of 11 
A

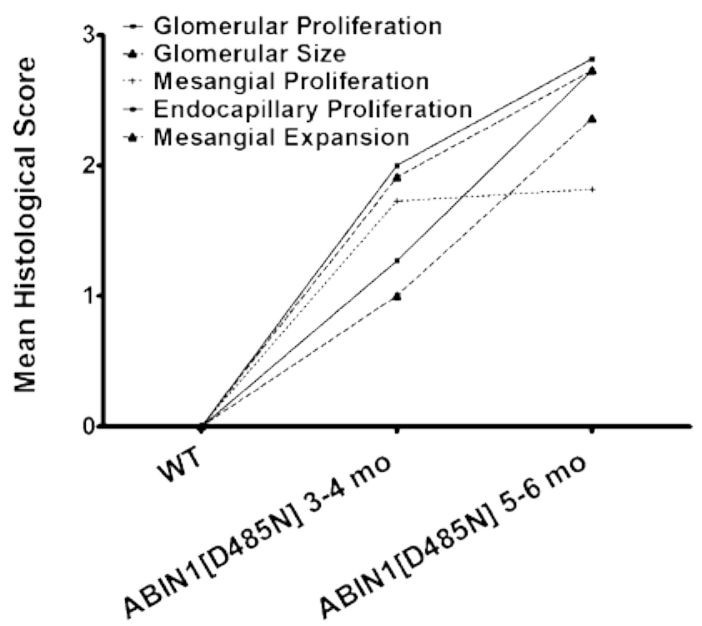

B

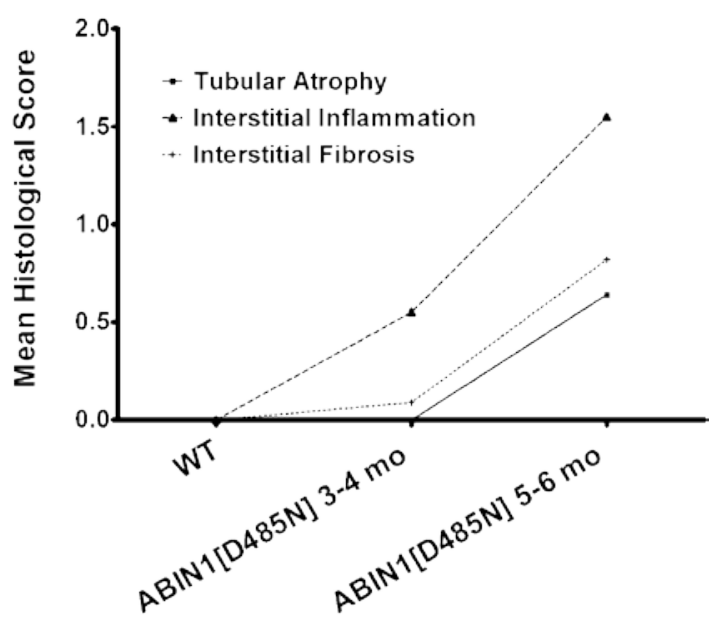

Figure 11 - ABIN1[D485N] Mice Develop Severe Progressive GN

Glomerular, interstitial, and tubular pathology was assessed by evaluation of a complete cross- section of an entire kidney in a blinded manner. Glomerular damage was assessed by semiquantitative scoring of size, cellularity, mesangial matrix expansion, crescent formation, and sclerosis on a 0 (normal), $1+$ (mild abnormality), 2+ (moderate abnormality), and 3+ (severe abnormality) scale. The semiquantitative scoring of interstitial inflammatory cell infiltration, interstitial fibrosis, and tubular atrophy was based on the percentage of interstitium area affected: 0 , normal; $1+,<25 \%$ of interstitial cross-section affected; $2+, 25 \%-50 \%$ of interstitium affected; $3+,>50 \%$ of interstitium affected in a single cross-section of an entire kidney. An activity index was calculated as the sum of individual scores for glomerular size, glomerular hyper- cellularity, cellular crescents, mesangial expansion, and interstitial inflammation. We also determined whether the lesions were focal (involving $<50 \%$ of the glomeruli) or diffuse (involving $>50 \%$ of the glomeruli). 
mice at 5-6 months. There were no differences in histologic injury between male and female mice.

Figure 12 shows that the urine albumin to creatinine (UAC) ratio was significantly elevated in 3-4 month old ABIN1[D485N] mice compared to WT. The urine albumin excretion did not increase further in 5-6 month old ABIN[D485N] mice. Surprisingly, serum C3 was found to be significantly higher in 3-4 month old ABIN1[D485N] animals compared to WT mice, but C3 was markedly decreased in the 56 month old ABIN1[D485N] mice (Figure 13). Our previous study showed that antinuclear antibodies were present by 3-4 months of age in ABIN1[D485N] mice [53].

The following figures include representative examples of the histology depicted in Table 6 and Figure 11. Figure 14b shows a glomerulus from an ABIN1[D485N] mouse at 4 months of age demonstrating mild mesangial and endocapillary hypercellularity with little or no mesangial matrix expansion or interstitial inflammatory infiltrate, and Figure 14a shows a glomerulus from a WT mouse for comparison. Figure 14c shows a glomerulus from a WT 6 month old mouse. Figure 14d, Figure 15, and Figure 16 show the more severe glomerular changes seen in 5-6 month old ABIN[D485N] mice. Figure 14d shows a glomerulus with moderate mesangial and endocapillary cell proliferation and a thickened capillary loop wall typical of “wire loops” (arrow). Figure 15 shows an example of severe glomerular hypercellularity, marked mesangial matrix expansion, and severe interstitial cell infiltration (arrow). Figure 16 shows a glomerulus with crescent formation (arrow), glomerular sclerosis, and interstitial fibrosis.

Figure 17a shows a transmission electron micrograph (TEM) from a 6 month old wild type mouse. Figure 17b shows a TEM from a 4 month old ABIN1[D485N] mouse 


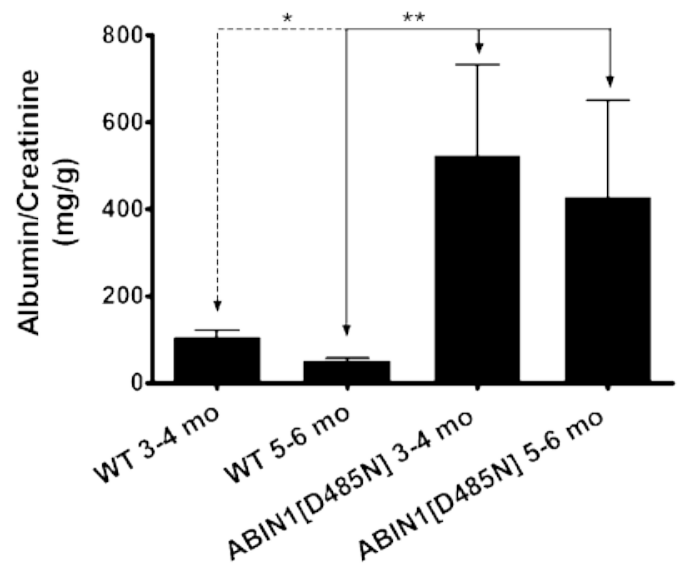

Figure 12 - Urine Albumin to Creatinine is Elevated in ABIN1[D485N] Mice

Urine albumin-to-creatinine ratio of 3- to 4-month-old and 5- to 6-month-old ABIN1[D485N] and WT mice (total $n=82$ ) show significant protein loss into the urine by 3-4 months of age in ABIN1[D485N] mice compared to ABIN1 WT. Significance was calculated using Mann-Whitney rank order analysis ( ${ }^{*} \mathrm{p}<0.05$ and $\left.{ }^{* *} \mathrm{p}<0.001\right)$.

Caster/Korte et al. 2013. 


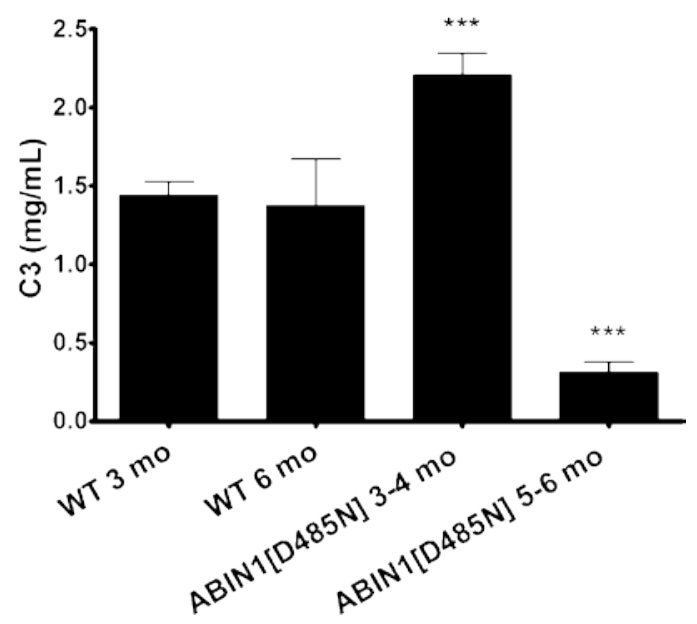

Figure 13 - Complement Component 3 Levels are Reduced in Late Disease

Reduced serum C3 levels are a clinical sign and diagnostic factor of SLE. Using the same cohort as that described in Figure 12, serum C3 levels were assessed and found to be lower than WT mice in older animals, although higher than WT in the younger animals. Significance was calculated using Mann-Whitney rank order analysis (*** $\mathrm{p}<0.0005)$.

Caster/Korte et al. 2013. 

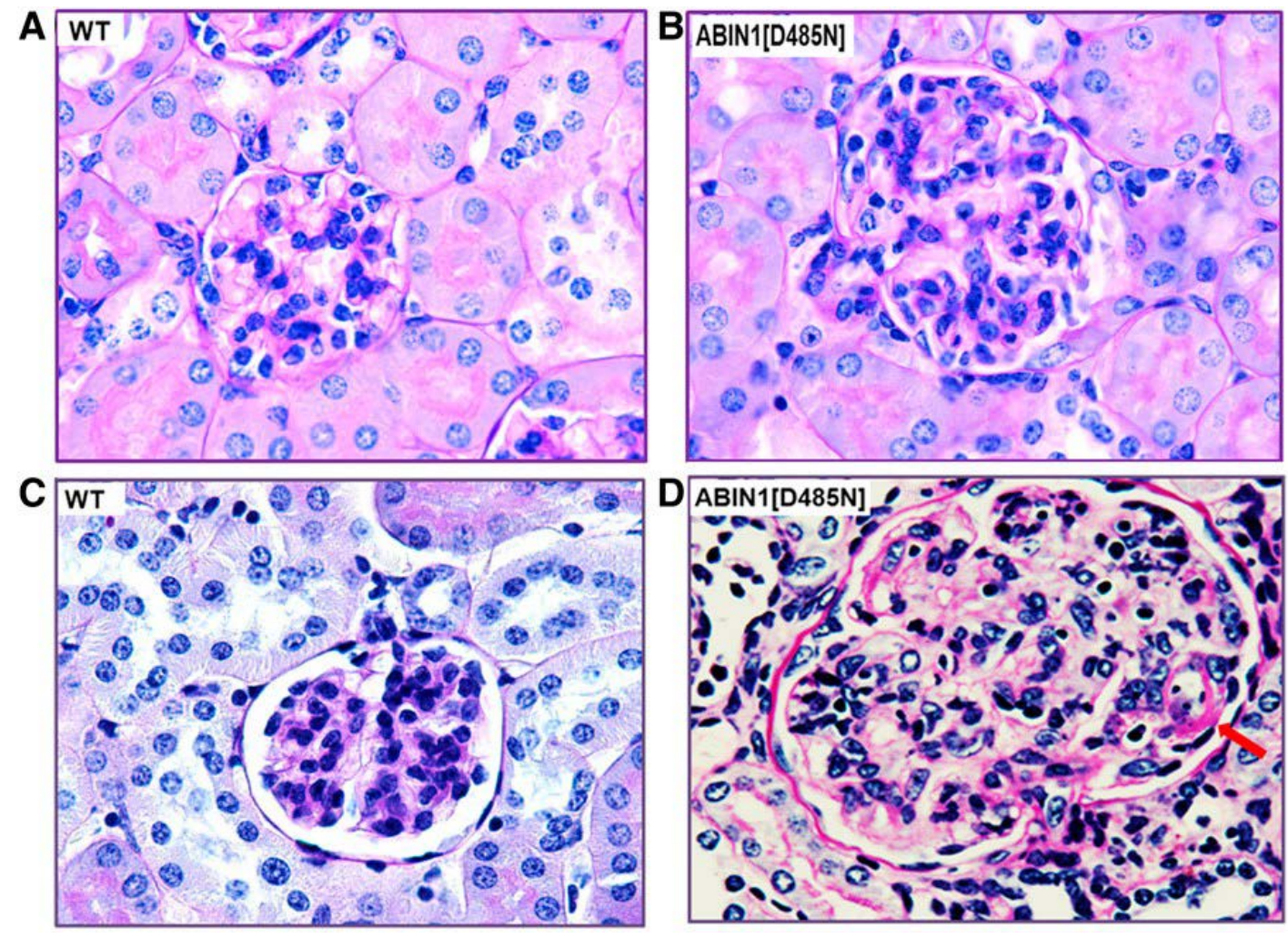

Figure 14 - Renal Histology of WT and ABIN1[D485N] Mice

Renal histology in wild-type and ABIN1[D485N] mice. (A) 100X magnification of a Periodic acid-Schiff (PAS) stained kidney section from a 3-4 month old wild-type (WT) mouse. (B) A comparative 100X PAS image shows that at 3-4 months the ABIN1[D485N] mouse kidneys display mesangial hypercellularity, matrix expansion and capillary loop thickening as compared to WT mouse kidneys. (C) 100X magnification of a Periodic acid-Schiff (PAS) stained kidney section from a 5-6 month old wild-type (WT) mouse. (D) A comparative $100 \mathrm{X}$ PAS image shows that at 5-6 months the ABIN1[D485N] mouse kidneys display severe mesangial hypercellularity, matrix expansion and "wire loops” (arrow) compared to WT mouse kidneys. 


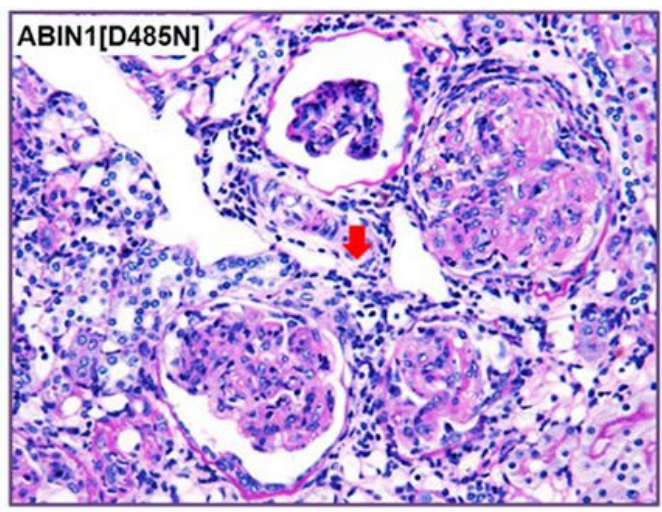

\section{Figure 15 - ABIN1[D485N] Mice Exhibit Interstitial Inflammation}

Representative 40X magnification PAS stain showing glomerular injury and extensive interstitial immune cell infiltration (arrow) observed in 5-6 month ABIN1[D485N] mouse kidneys.

Caster/Korte et al. 2013. 


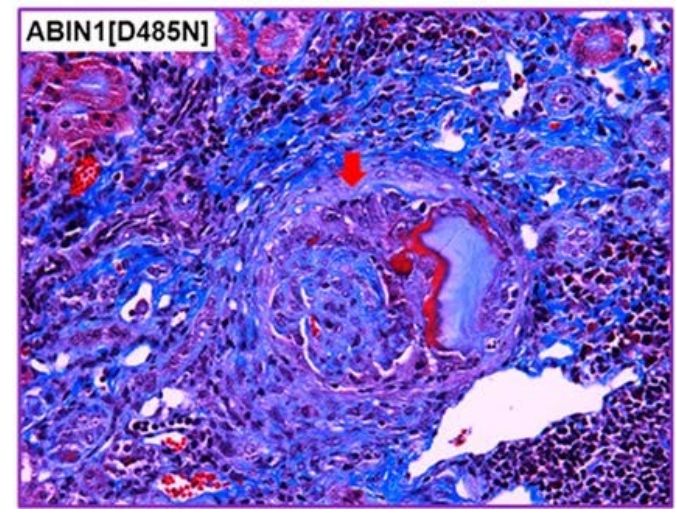

Figure 16 - ABIN1[D485N] Mice Exhibit Interstitial Fibrosis

Representative 40X magnification of Masson's trichrome staining showing examples of tubuleinterstitial fibrosis, glomerular fibrosis and crescent formation (arrow) and immune cell infiltration (lower right corner) in 5-6 month ABIN1[D485N] mouse kidneys.

Caster/Korte et al. 2013. 

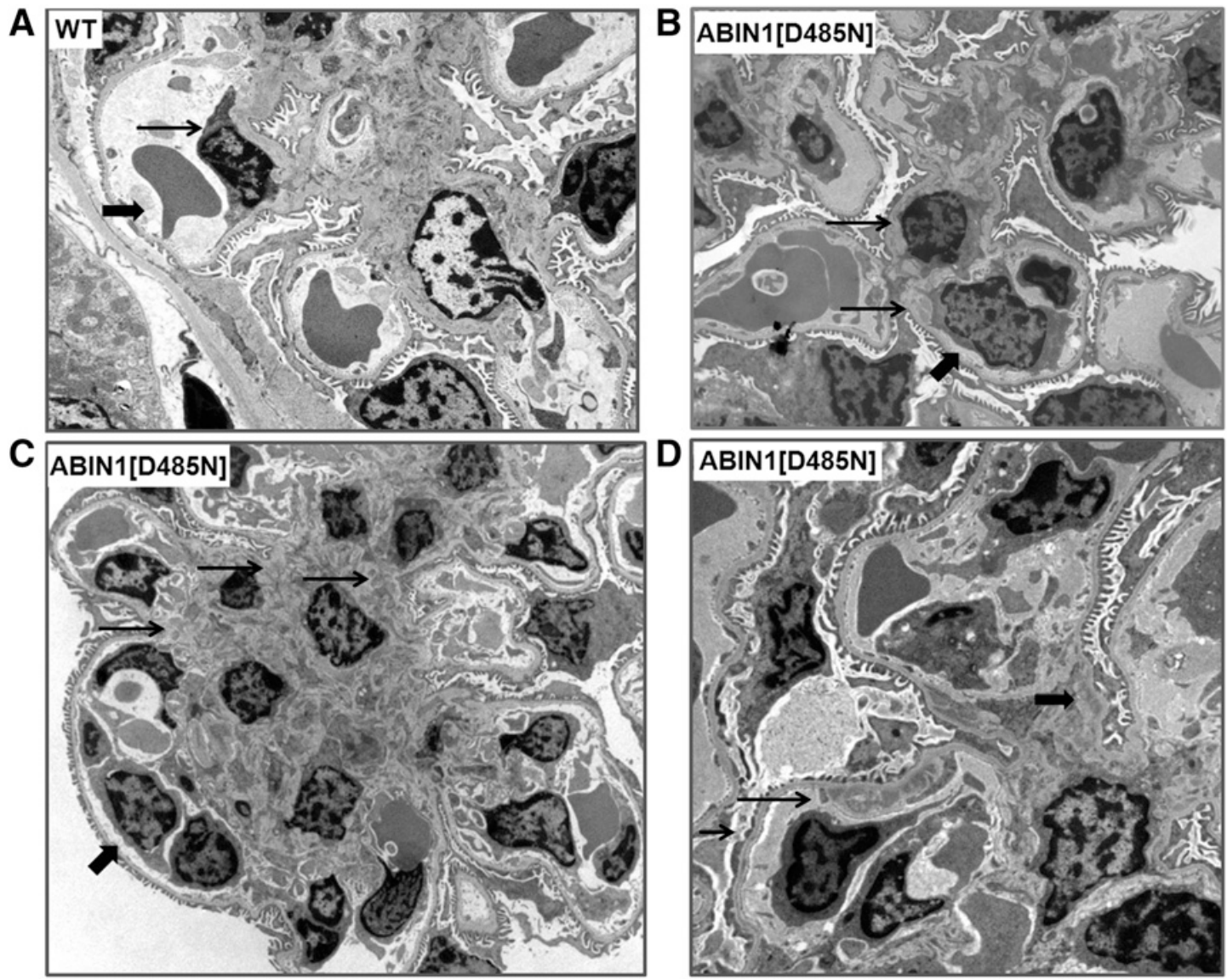

Figure 17 - ABIN1[D485N] mouse glomeruli display mesangial and subendothelial deposits.

(A) TEM image of WT mouse kidney (large arrow, erythrocyte; small arrow, endothelial cell). (B) Minimal mesangial expansion and immune deposits (small arrows) in a TEM image of a 4-month-old ABIN1[D485N] mouse kidney. Podocyte foot processes are intact. The large arrow points out a monocyte in a capillary. (C) Extensive mesangial expansion and immune deposits (small arrows) in a TEM image of a 6-month-old ABIN1[D485N] mouse kidney. The large arrow points out a monocyte in a capillary. (D) Example of subendothelial immune deposits (long small arrow), areas of podocyte effacement (short small arrow), and mesangial immunoglobulin deposits (large arrow) in a 6-month-old ABIN1[D485N] mouse kidney. Caster/Korte et al. 2013. 
demonstrating moderate mesangial expansion and small electron dense deposits in the mesangium (small arrows). Podocyte foot processes are intact. The large arrow points out a monocyte in a capillary. TEM in Figure 17c shows extensive mesangial expansion and mesangial deposits in a 6 month old ABIN1[D485N] mouse kidney (small arrows). The large arrow points out a monocyte in a capillary. Figure 17d shows a TEM from a 6 month old ABIN1[D485N] mouse demonstrating subendothelial (long, small arrow) and mesangial (large arrow) electron dense deposits and focal foot process effacement (short, small arrow).

Figure 18 shows immunofluorescence (IF) images of 5-6 month ABIN1[D485N] kidneys demonstrating IgG, IgA, IgM, C3, and C1q deposition along capillary loops and in the mesagium. IF from 3-4 month old ABIN1[D485N] mouse kidneys showed mild deposition of immunoglobulin and complement within the mesangium (not shown).

Our results show that loss of ABIN1 function in NF- $\mathrm{BB}$ activity is associated with the development of proteinuria and proliferative glomerulonephritis with features typical of class III and IV LN. Those changes become progressively worse between 3 and 6 months, at which time they are associated with low serum C3 levels. From our animal

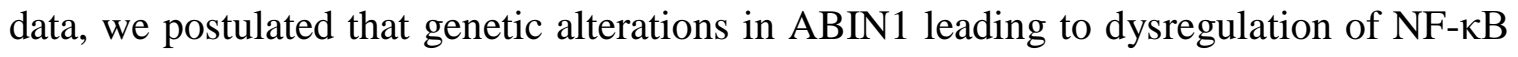
and MAPK activation could be involved in the development of LN. This hypothesis led us to evaluate SNPs in the gene for ABIN1, TNIP1, in SLE patients with and without LN.

\section{Association of TNIP1 SNPs and Human Lupus Nephritis}

To investigate a clinical relevance for ABIN1 dysfunction in human lupus nephritis (LN), 5 SNPs in TNIP1 previously associated with autoimmune diseases, including SLE, were 


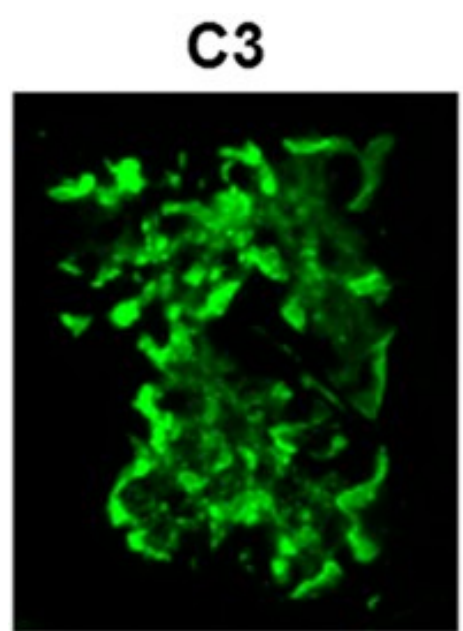

$\lg \mathrm{A}$

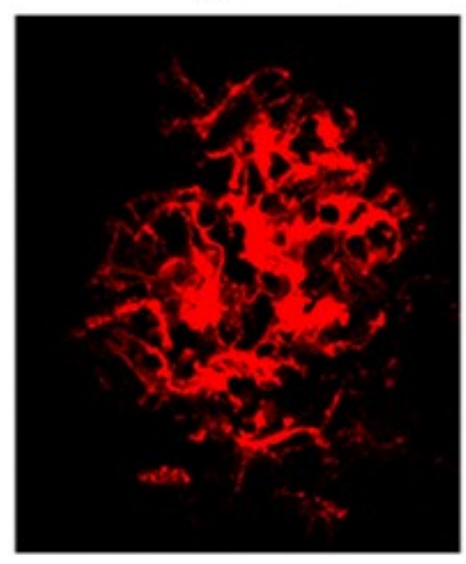

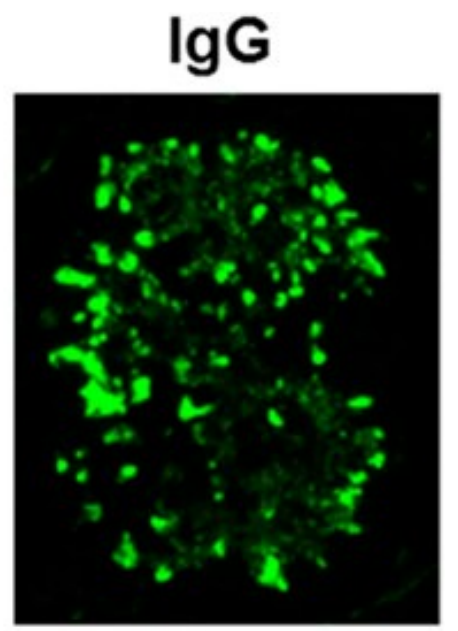

\section{$\lg M$}

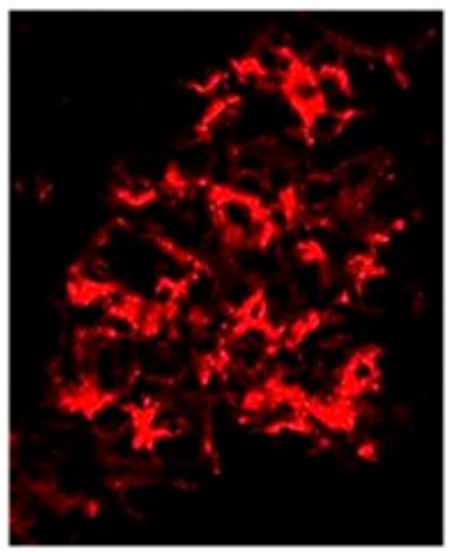

Figure 18 - Deposits in ABIN1[D485N] glomeruli contain complement factors and immunoglobulins.

The panels show examples of the immunofluorescence staining that was observed for the

different labeled complement factors and immunoglobulin subtypes in kidneys from 5 to 6-month-old ABIN1[D485N] mice. No significant immunofluorescence was observed for age-matched WT mice.

Caster/Korte et al. 2013. 
genotyped in a total of 16,999 individuals of European-ancestry (EA), African American (AA), Asian, Gullah and Hispanic as described in Chapter 2 (Table 7) ([65]). After applying quality control assessment, we studied 15,864 individuals consisting of 3,089 SLE cases with LN, 4,308 SLE cases without LN, 1,097 SLE cases with unknown status of LN, and 7,492 healthy controls (Table 8). To evaluate evidence for genetic association, we performed single-marker logistic regression analysis adjusting for gender and global ancestry estimates. Comparing SLE cases with lupus nephritis to SLE cases without LN (case/case analysis) showed strong associations surpassing the Bonferroni corrected significant threshold $P<0.01$ at rs7708392 in EAs $\left(P=3.66 \times 10^{-4}\right.$, odds ratio $(\mathrm{OR})=$ 1.22) and rs4958881 in AAs $\left(P=8.47 \times 10^{-3}, \mathrm{OR}=1.22\right)$ (Table 9). No SNPs reached the Bonferroni corrected significant threshold in other populations. Analysis of SLE cases with LN versus healthy control subjects confirmed the validity of our case only analysis (rs7708392 in EAs: $P=1.82 \times 10^{-11}$, OR $=1.44$; rs4958881 in AAs: $P=4.43 \times 10^{-3}$, OR $=1.20)$. Overall, our results indicate that polymorphisms in the region of TNIP1 are associated with LN in EAs and AAs.

\section{$\underline{4.3-\text { Discussion }}$}

Here I show that a knock-in mouse expressing an ABIN1 mutant, ABIN1[D485N], with impaired negative regulation of $\mathrm{NF}-\kappa \mathrm{B}$, developed progressive, proliferative glomerulonephritis with histologic features of class III and IV human lupus nephritis (LN), and 2 SNPs of the ABIN1 gene, TNIP1, are significantly associated with 
Table 7 - Sample summary before quality control adjustments.

\begin{tabular}{|c|c|c|c|c|c|c|c|c|}
\hline 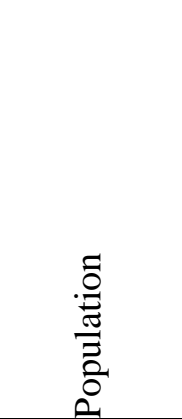 & 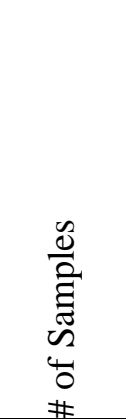 & 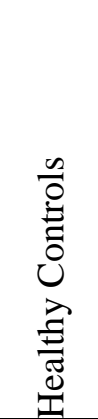 & 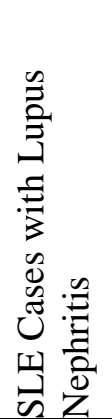 & 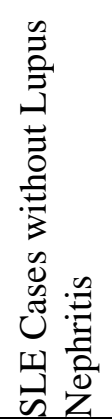 & 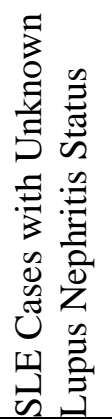 & $\frac{0}{\frac{\pi}{\pi}}$ & 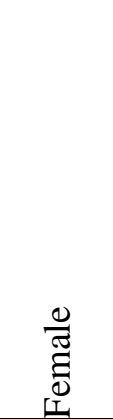 & 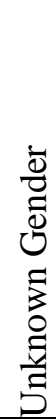 \\
\hline $\begin{array}{l}\text { European } \\
\text { American }\end{array}$ & 8066 & 3818 & 1243 & 2306 & 699 & 1642 & 6403 & 21 \\
\hline $\begin{array}{l}\text { African- } \\
\text { American }\end{array}$ & 3462 & 1893 & 636 & 663 & 270 & 723 & 2733 & 6 \\
\hline Asian & 2676 & 1348 & 561 & 633 & 134 & 296 & 2380 & 0 \\
\hline Gullah & 286 & 131 & 70 & 80 & 5 & 34 & 252 & 0 \\
\hline Hispanic & 2509 & 887 & 702 & 823 & 97 & 227 & 2279 & 3 \\
\hline Total & 16999 & 8077 & 3212 & 4505 & 1205 & 2922 & 14051 & 30 \\
\hline
\end{tabular}

Caster/Korte et al. 2013. 
Table 8 - Sample Summary Following Quality Control Adjustments

\begin{tabular}{|c|c|c|c|c|c|c|c|}
\hline 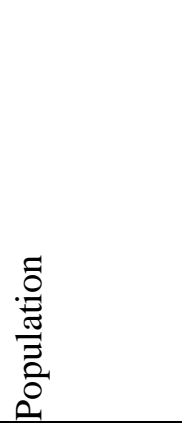 & 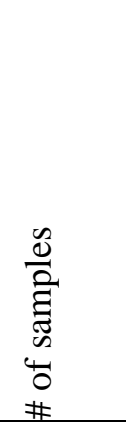 & 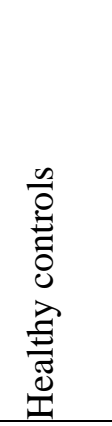 & 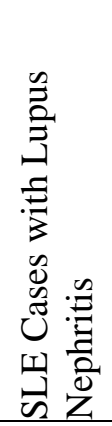 & 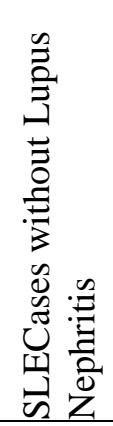 & 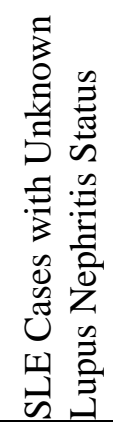 & $\frac{0}{\pi}$ & 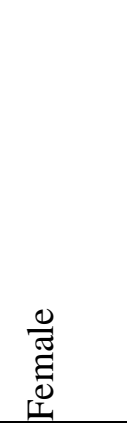 \\
\hline $\begin{array}{l}\text { European- } \\
\text { American }\end{array}$ & 7427 & 3491 & 1129 & 2161 & 646 & 1495 & 5932 \\
\hline $\begin{array}{l}\text { African- } \\
\text { American }\end{array}$ & 3338 & 1811 & 709 & 704 & 234 & 695 & 2643 \\
\hline Asian & 2525 & 1260 & 529 & 610 & 126 & 253 & 2272 \\
\hline Gullah & 275 & 123 & 70 & 79 & 3 & 33 & 242 \\
\hline Hispanic & 2299 & 807 & 652 & 754 & 88 & 207 & 2092 \\
\hline Total & 15864 & 7492 & 3089 & 4308 & 1097 & 2683 & 13181 \\
\hline
\end{tabular}

Caster/Korte et al. 2013. 


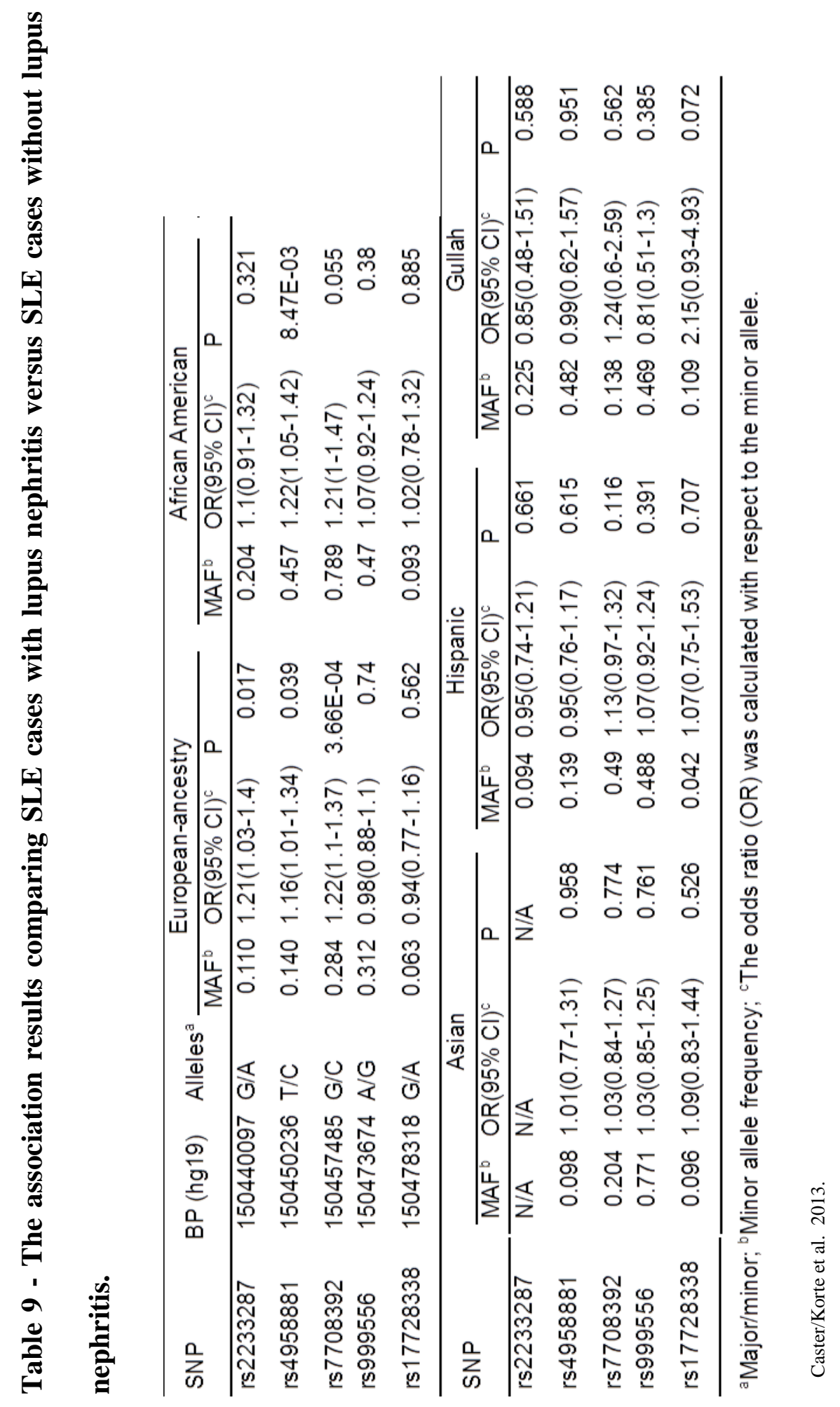


human LN. A role for NF- $\mathrm{BB}$ in the incidence and severity of LN was suggested previously by the correlation of enhanced NF- $\kappa$ B protein expression in disease kidney sections or reduction of disease phenotypes in animal models after administration of compounds that indirectly inhibit NF- $\mathrm{B}$ signaling [30-34]. Although the mechanisms regulating those effects of NF- $\kappa \mathrm{B}$ on $\mathrm{LN}$ have not been determined, these data provide a potential mechanism for NF-кB dysregulation in some patients with LN.

K63 and linear polyubiquitin conjugation mediates a number of protein-protein

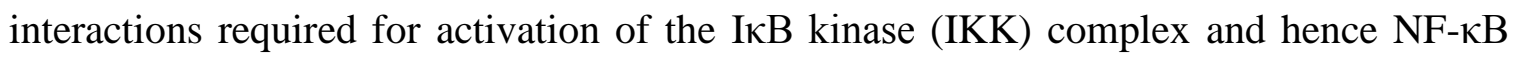
transcription [56,174-176]. ABIN1 contain a polyubiquitin binding domain (UBD) that is

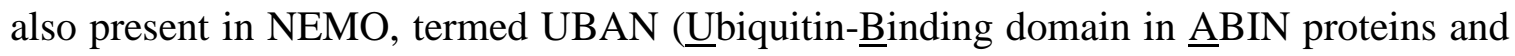
NEMO) [62]. . The UBAN in ABIN1 contains a highly conserved region consisting of amino acids 472-480 in humans and 485-495 in mice [62]. The Asp to Asn mutation at this conserved residue 485 (mouse) or 472 (human) of the UBAN renders ABIN1 incapable of binding recombinant Lys63 and linear polyubiquitin chains and polyubiquitinated NF- $\kappa$ B activators from cell lysates [44,50,51,53,62]. We previously reported that ABIN1[D485N] knock-in mice have enhanced NF- $\mathrm{B}$ signaling in B cells and bone marrow-derived macrophages and developed a SLE-like autoimmune disease, with enlarged spleens and lymph nodes, elevated levels of pathogenic immunoglobulins, and antinuclear antibodies in the serum as early as 4 months of age [53]. The present report shows that those mice develop proteinuria by 3-4 months of age, hypocomplementemia by 5-6 months, and renal histologic abnormalities including focal and diffuse glomerular hypercellularity involving both endocapillary and mesangial cells, increased mesangial matrix accumulation, “wire loop” thickening of glomerular capillary 
walls, immunoglobulin (IgG, IgA, and IgM) and complement deposition in a mesangial and capillary loop pattern, and mesangial and subendothelial electron dense deposits by electron microscopy. Those findings suggest that ABIN1[D485N] knock-in mice provide a model of human SLE, including the development of class III/IV LN. Our model indicates that SLE and LN can be initiated by disruption of ABIN1 NF- $\kappa B$ inhibitory function, at least in part, in B cells. Another recent report showed that ABIN1-deficient mice develop progressive lupus-like autoimmune phenotypes and glomerulonephritis [52]. In addition to NF- $\kappa$ B activation, we also showed in a previous report that JNK and p38 MAPK signaling was enhanced after stimulation with TLR agonists in B cells, bone marrow-derived macrophages (BMM), and dendritic cells isolated from ABIN1[D485N] mice [53]. A role for enhanced MAPK signalling in the development of glomerulonephritis (GN) has been suggested in several reports [177-180]. Stambe et al. showed that there was enhanced active p-p38 MAPK in glomeruli, tubules, myofibroblast and infiltrated neutrophils and macrophage in kidney biopsies from patients with different types of proliferative GN, including class III/IV LN and that elevated p-p38 MAPK correlated with renal dysfunction and histopathology [180]. The same group showed in a separate study that administration of a specific p38 MAPK inhibitor prevented renal injury and renal function loss from anti-glomerular basement membrane (GBM)-induced GN in rats [179]. Another report showed that proteinuria and glomerular cell proliferation-induced by anti-GBM was dependent on JNK activity in bone-marrow macrophages [177]. Taken together, the present study and the previous reports support the hypothesis that loss of ABIN1 K63 and linear polyubiquitin binding results in

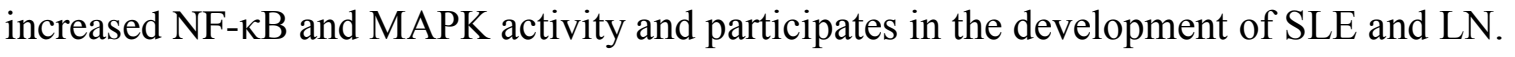


Variants in the ABIN1 gene TNIP1 have been reported in SLE patients, suggesting a role for ABIN1 in human autoimmune disease [65,67,68]. Gateva et al. showed an association for a TNIP1 variant (rs7708392) in European-Ancestry American and Swedish SLE patients [67]. Han et al. also reported a TNIP1 variant (rs10036748) in a Chinese Han SLE population [68]. This SNP also showed significant association with European population, and the frequency (77\%) was much higher in the European population than in the Chinese population (26\%) [68]. Both of these variants are in noncoding regions of TNIP1, and the functional consequence and association with LN were not reported. The associations of those TNIP1 variants were also replicated in Japanese (rs7708392) and Chinese Han (rs10036748) populations [181,182]. There was a stronger association for rs7708392 with LN in the Japanese population [181]. A replicate study of rs7708392 and rs10036748 in a southwest Chinese SLE population found a weak association, but as for the Japanese population, a significant association for rs7708392 with LN in their SLE population was apparent [69]. Consistent with those previous reports, the current study found association $\left(P=3.66 \times 10^{-4}\right.$, odds ratio $\left.(\mathrm{OR})=1.22\right)$ for rs7708392 when comparing American SLE patients of European-Ancestry with LN vs. those without LN, but this association was not significant in African, Asian or Hispanic American cohorts. The discord for the finding in our Asian cohort and the reported finding in southwest Chinese and Japanese populations with regards to LN association may be explained by the composition of our Asian population of 1012 total patients, which was $90 \%$ Korean. The present report also identified association for another TNIP1 variant (rs4958881) $\left(P=8.47 \times 10^{-3}\right.$, OR = 1.22) in African American SLE patients with LN vs. those without LN. rs4958881 was previously reported to be associated with 
systemic sclerosis (SSc), but not SLE [71]. Additionally, ABIN1 expression was decreased in SSc skin lesions and in dermal fibroblast from SSc patients, compared to controls, and transgenic expression of ABIN1 abrogated matrix protein expression induced by inflammatory cytokines in fibroblast from SSc patients [71]. This suggests that the rs4958881 variant could result in lower renal expression of ABIN1 in LN, leading to increased mesangial cell matrix production typically seen in class III and IV LN in humans [10] and found in the ABIN1[D485N] knock-in mice.

In summary, these data suggest that mutations in ABIN1 represent a new genetic basis for LN through the novel pathophysiologic mechanism of aberrant regulation of NF- $\mathrm{kB}$ and MAPK activity (Figure 19). This is supported by knock-in mice expressing a mutation of ABIN1 with loss of NF-KB and MAPK inhibitory function and a strong association of a novel TNIP1 polymorphism and LN in a SLE patient population with a higher incidence of LN. Our findings support an association between polymorphisms in TNIP1 and the development of LN. This mouse model closely replicates the human disease and, therefore, potentially serves as an effective tool to study diagnostic and therapeutic strategies for LN. 


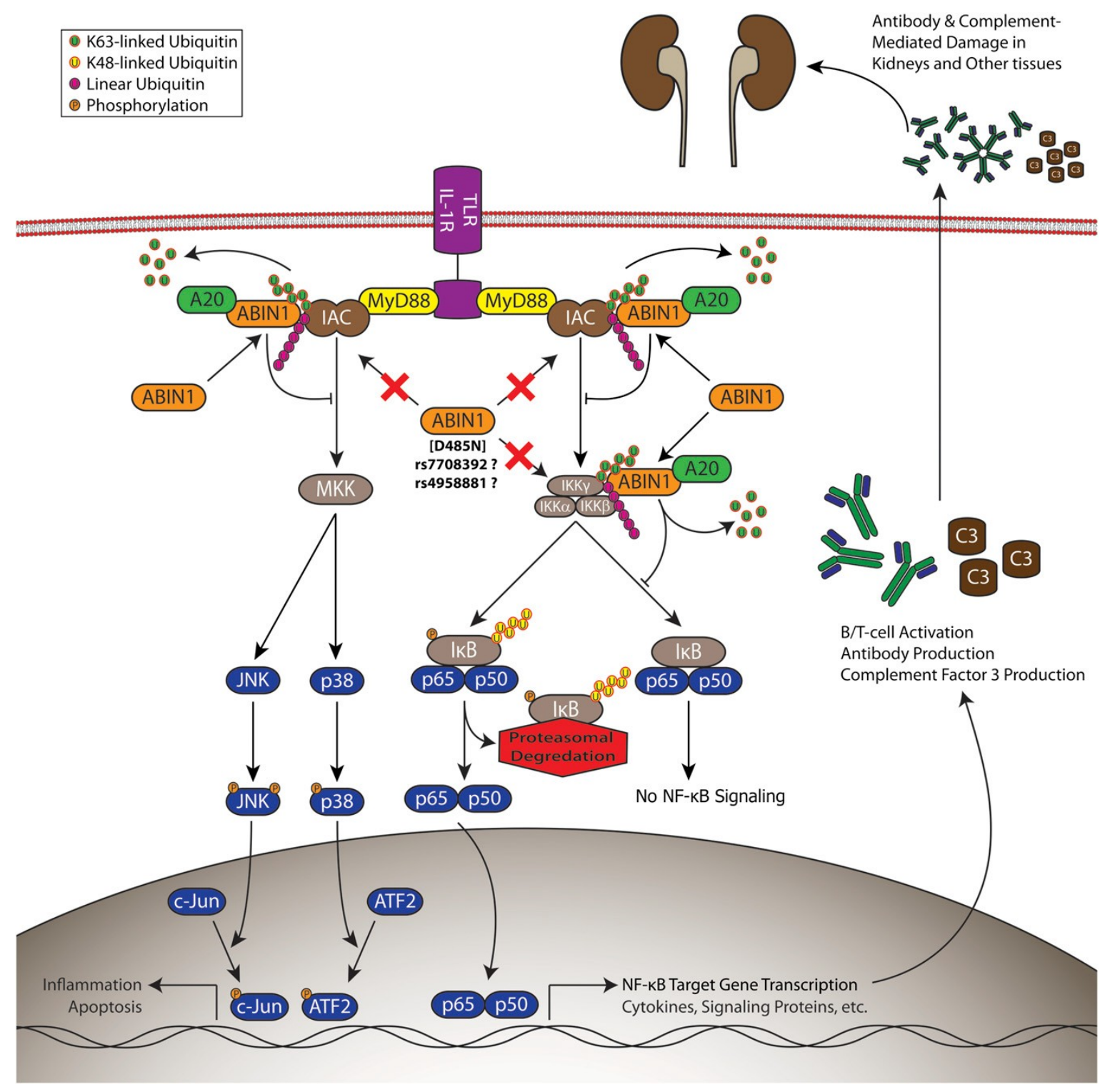

Figure 19 - ABIN1 aberrant regulation of NF-אB and MAPK signaling in LN.

Pro-inflammatory gene activation is mediated by activation of the upstream I kappa B kinase (IKK) complex or MAPK kinases (MKK) activation of JNK or p38 MAPK. IKK and MKKs are activated by an Inflammatory Activating Complex (IAC) consisting of IRAKs, TRAFs, RIPs, and TAK1, among others. ABIN1 binds to K63-linked and linear polyubiquitin chains and inhibits components of the IAC or IKK $\gamma$. 


\section{CHAPTER V}

\section{ABIN1 DETERMINES THE SEVERITY OF ANTIBODY-MEDIATED GLOMERULONEPHRITIS THROUGH A NOVEL PODOCYTE-NEUTROPHIL AXIS}

\section{$\underline{5.1-\text { Introduction }}$}

Proliferative glomerulonephritis (GN) is an important cause of end-stage renal failure [183]. The current paradigm for the pathogenesis of GN is that immunoglobulin deposition in the glomerulus, in the form of immune complexes or autoantibodies against constituents of the glomerulus, initiates a proinflammatory response through Fc receptor (FcR) ligation and activation of the complement cascade, leading to immune cell recruitment and activation. Mesangial cells respond to immunoglobulin and complement by regulating the glomerular microenvironment through the production of various cytokines and chemokines, resulting in increased endothelial cell expression of adhesion molecules that recruit and activate leukocytes [15,18]. Although inflammatory injury leads to podocyte dysfunction, the response of podocytes to immunoglobulin and/or complement deposition and their contribution to the inflammatory response are poorly understood, and only a few authors have published work to expand this field [22,23]. Although limited in number, these studies suggest that podocytes have the potential to 
actively participate in the modulation of the immune response. If podocytes can be shown to provide the cytokines and chemokines that influence glomerular inflammation, this may open up therapeutic avenues never before considered.

It is well established that neutrophils are pathogenic protagonists in antibodymediated GN, although recent effort has focused on cells that function in a more classic immunoregulatory role, such as monocytes, B-cells, T-cells, and dendritic cells [23-29]. Neutrophil granules contain both reactive oxygen species and proteases that cause injury to vascular tissue when released, and the glomerulus is in the unique situation to be constantly patrolled by neutrophils [25-28]. Still, direct injury of podocytes by neutrophils has not previously been evaluated.

As highlighted previously, NF- $\mathrm{BB}$ activation is associated with GN and is important to podocyte response to proinflammatory stimulation [35-37]. Inhibition of $\mathrm{NF}-\kappa \mathrm{B}$ signaling protects against development of disease, at least in part, through reduced expression of cytokines that are transcriptional targets of NF- $\kappa \mathrm{B}[30,33,34,38]$. Chapter IV of this dissertation as well as previous work describe ABIN1 as an important inhibitor of NF- $\mathrm{BB}$ activation $[54,55,59]$. It characterizes mice expressing the ubiquitin bindingdeficient mutant, ABIN1[D485N], as prone to spontaneous immune hyperactivation and severe GN, which is frequently fatal by 6 months $[53,184]$. The work outlined in Chapter IV describes a pathogenic state in which ABIN1 fails to inhibit NF- $\kappa B$ stimulation to allow rampant hyperactivation and uncontrolled inflammation. It did not define a specific cellular mechanism or tissue-specific interaction which could be a target for therapeutic pharmacologic intervention, or allow current therapies to be better targeted to affected tissues for reduced side effects. 
The present study used nephrotoxic sera (NTS) containing antibodies directed against the glomerular basement membrane (GBM) to induce a well-characterized model of nephrotoxic nephritis (NTN). NTS-induced damage in wild-type and ABIN1[D485N] mice is used to address the hypothesis that NF-кB-dependent proinflammatory mediator production by intrinsic renal cells, particularly podocytes, contributes to initiation and progression of GN through enhanced retention and activation of neutrophils. The model of acute NTN mimics the pathogenesis of a number of human glomerular diseases, including lupus nephritis and rapidly progressive GN, and anti-GBM GN is associated

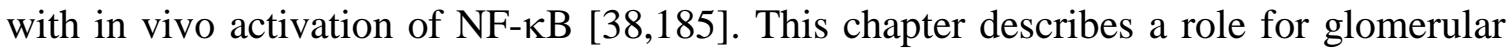
cell ABIN1 regulation of NF- $\kappa B$ in antibody-mediated GN, and we describe a novel pathogenic interaction between podocytes and neutrophils within the glomerulus.

\section{$\underline{5.2 \text { - Results }}$}

\section{ABIN1[D485N] mice exhibit exaggerated and localized renal disease compared to WT}

Chapter IV describes that ABIN1[D485N] mice spontaneously develop severe GN beginning at 3-4 months of age that progresses by 5-6 months [184]. To define the effects for this ABIN1 mutation on intrinsic glomerular cells, NTS-induced injury was induced at 6 weeks of age, immediately after weaning. Figure 20 shows urine albumin:creatinine (mg/g) ratios (UAC) from ABIN1[D485N] and WT mice obtained 


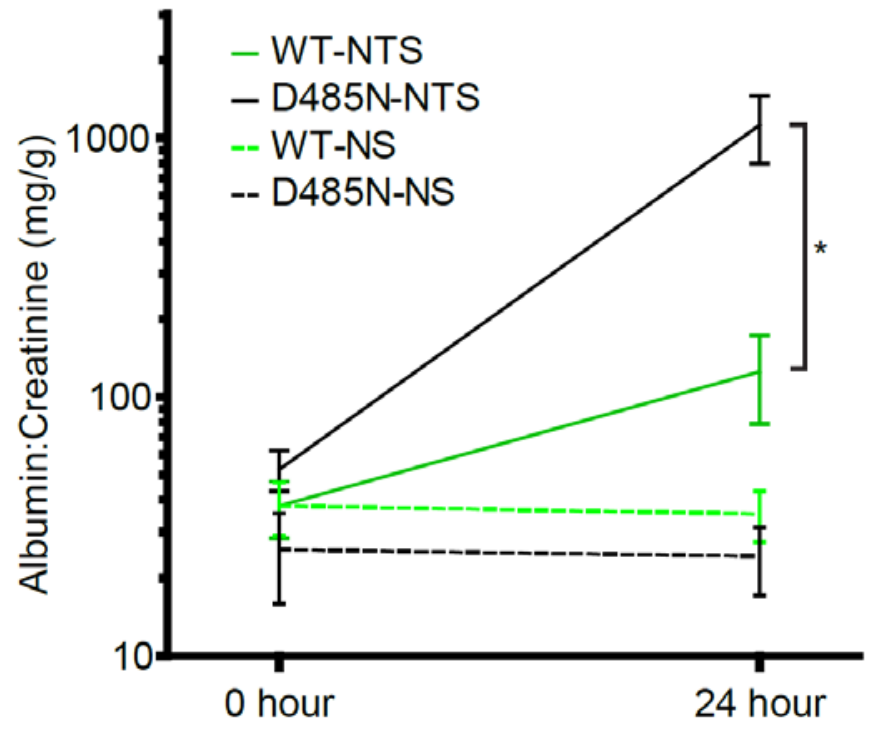

Figure 20 - ABIN1[D485N] Mice More Sensitive to NTS than WT.

NTS administered to mice at 6 weeks of produces significantly different levels of proteinuria, with ABIN1[D485N] animals exhibiting 9x the level of urine albumin. Normal serum (NS) served as a control and did not induce proteinuria. Injection of ontrol sera (NS), did not change proteinuria in either WT or ABIN1[D485N] mice. $(n=6-12$ per condition) Student's T-test was used to compare values between two groups. 
just prior to NTS tail vein injection (0 hour) and 24 hours after NTS injection. The 0 hour UAC of WT and ABIN1[D485N] mice were not different, and administration of an equal amount of normal serum immunoglobulin did not increase the 24 hour UAC above unstimulated levels in either WT or ABIN1[D485N] mice. Both WT and ABIN1[D485N] mice demonstrated a significant increase in UAC 24 hours after NTS administration, but the increase in UAC was 9-fold higher in ABIN1[D485N] mice compared to WT mice.

To separate the effects of ABIN1[D485N] on systemic immune activation and on intrinsic renal cells, NTN was induced in chimeric mice, as described in materials and methods. Figure 21 shows that NTS-induced proteinuria was similar in WT mice whether transplanted with WT or ABIN1[D485N] mouse bone marrow. Again, as shown in Figure 20, ABIN1[D485N] mice showed significantly greater albuminuria than wildtype mice, and that proteinuria was similar in ABIN1[D485N] mice transplanted with WT or ABIN1[D485N] mouse bone. Thus, differences in proteinuria in chimeric mice were due to the host genotype, not to the immune system genotype. This conclusion was supported by serum expression of common immune mediators. Figure 22 shows that no significant differences between WT and ABIN1[D485N] mice in serum levels of G-CSF, GM-CSF, IL1 $\alpha / \beta$, IL2, IL3, IL4, IL5, IL6, IL12p70/p40, TNF- $\alpha$, KC, MCP-1, MIP1 $\alpha / \beta$, or RANTES were observed.

To examine the local glomerular inflammatory response to NTS, the number of CD45 and myeloperoxidase (MPO) positive leukocytes infiltrating glomeruli was determined at $2 \mathrm{hr}$ and $24 \mathrm{hr}$ after NTS administration by immunohistochemistry (IHC). CD45 is expressed on all leukocytes, while myeloperoxidase (MPO) is highly expressed 


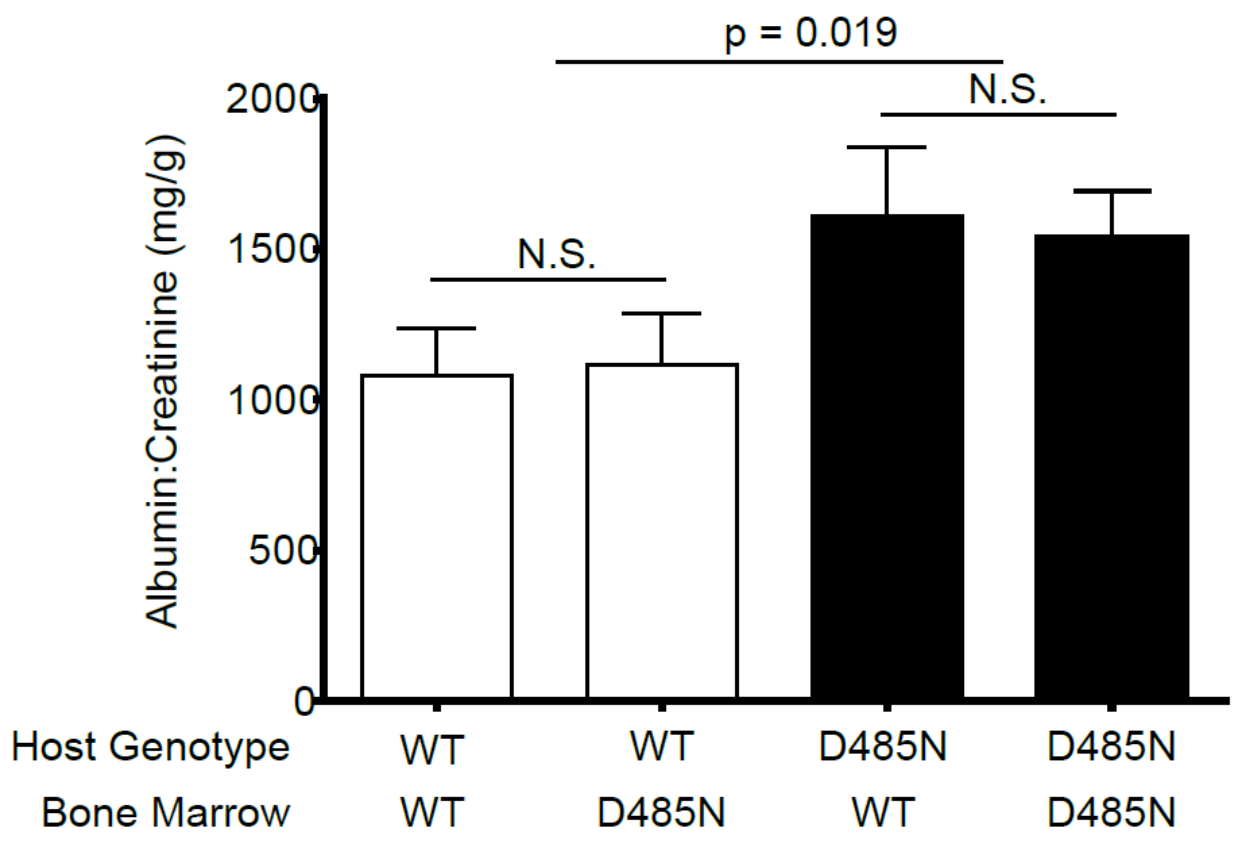

Figure 21 - Bone Marrow Transplant Suggests Host Genotype is an Important Factor in Response to NTS

Bone marrow transplant using ABIN1 WT hosts and either WT or ABIN1[D485N] bone marrow ( $\mathrm{n}=10$ each) produced no change in proteinuria at 13 weeks 24 hours post-NTS. Likewise, ABIN1[D485N] hosts did not respond differently due to immune genotypes ( $n=5$ each), however the host genotype was related to a significant change in proteinuria severity following NTS administration. ANOVA was used to compare values between the 4 conditions.

Korte, unpublished. 


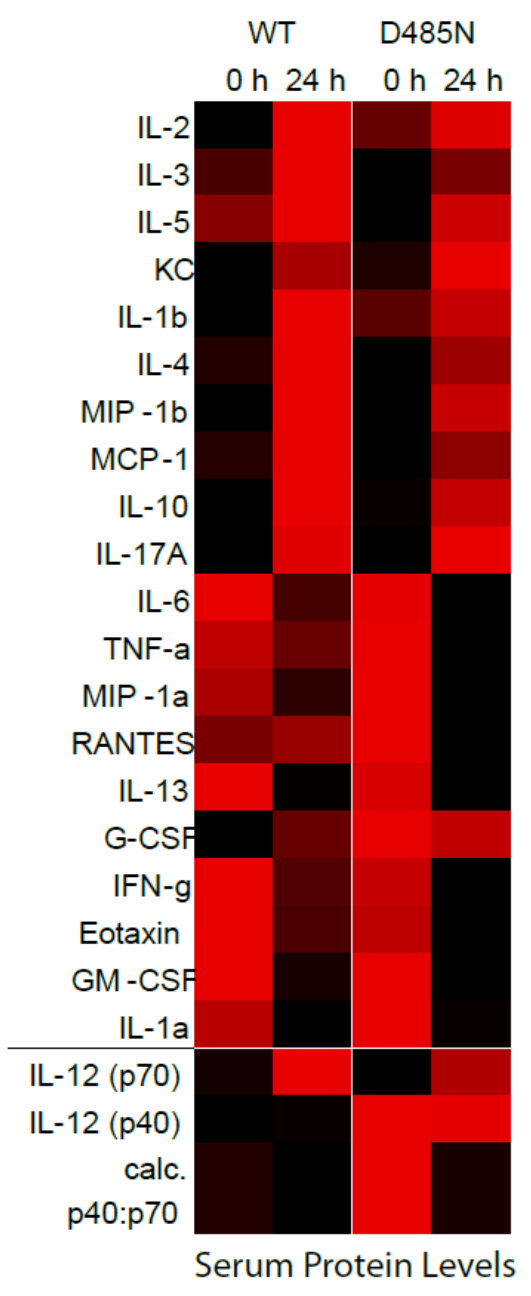

Figure 22 - Serum Cytokine Analysis of WT and ABIN1[D485N] Mice before and following NTS

Serum cytokine expression was relatively unchanged between ABIN1 WT and ABIN1[D485N] animals. Black corresponds to lower relative expression and increasing red corresponds to increasing expression of an individual cytokine within the four conditions. ( $\mathrm{n}=4$ per condition)

Korte, unpublished. 
in neutrophil azurophilic granules, modestly expressed in monocyte lysosomes, and lost from tissue macrophages[186]. At 2 hours, WT mice expressed an average $( \pm 95 \%$ confidence interval) of $1.44( \pm 0.28)$ CD45 positive cells per glomerulus while ABIN1[D485N] mice had $2.31( \pm 0.12)$ (Figure 23). At 24 hours, WT mice glomeruli decreased to $1.10( \pm 0.36)$ CD45 positive cells per glomeruli while ABIN1[D485N] increased slightly to $2.33( \pm 0.29)$ cells per glomeruli (Figure 23). Likewise, WT mice at 2 hours expressed $0.60( \pm 0.13)$ MPO positive cells per glomerulus while ABIN1[D485N] mice had more than twice the MPO positive cells with 1.34 ( \pm 0.19$)$ (Figure 23). At 24 hours, MPO positivity of WT mice glomeruli descreased to $0.23( \pm 0.11)$ MPO positive cells while ABIN1[D485N] maintained 0.95 $( \pm 0.35)$ cells per glomeruli (Fig. 1e, p > 0.05). Representative images are shown in Figure 24.

Structural and ultrastructural characteristics of NTS challenged ABIN1[D485N] and WT mice were assessed by light microscopy, transmission electron microscopy (TEM), and WT-1 IHC. Blinded analysis of Periodic acid-Schiff (PAS) stained kidney sections showed no differences in mesangial cellularity between WT and ABIN1[D485N] mice that received control serum or NTS ( $\mathrm{n}=5$ per condition) (Figure 25 and Figure 26). Mesangial matrix was significantly increased only in ABIN1[D485N] mice that received NTS. Endothelial cell and podocyte changes were assessed using TEM (Figure 27). Endothelial cells show a typical ultrastructure without obvious signs of pathology. Podocytes showed prominent foot process effacement and slit diaphragm loss that was diffuse in ABIN1[D485N] mice, but focal in WT mice. Glomerular expression of the podocyte nuclear marker, WT-1, typically decreases in GN, and that decrease 

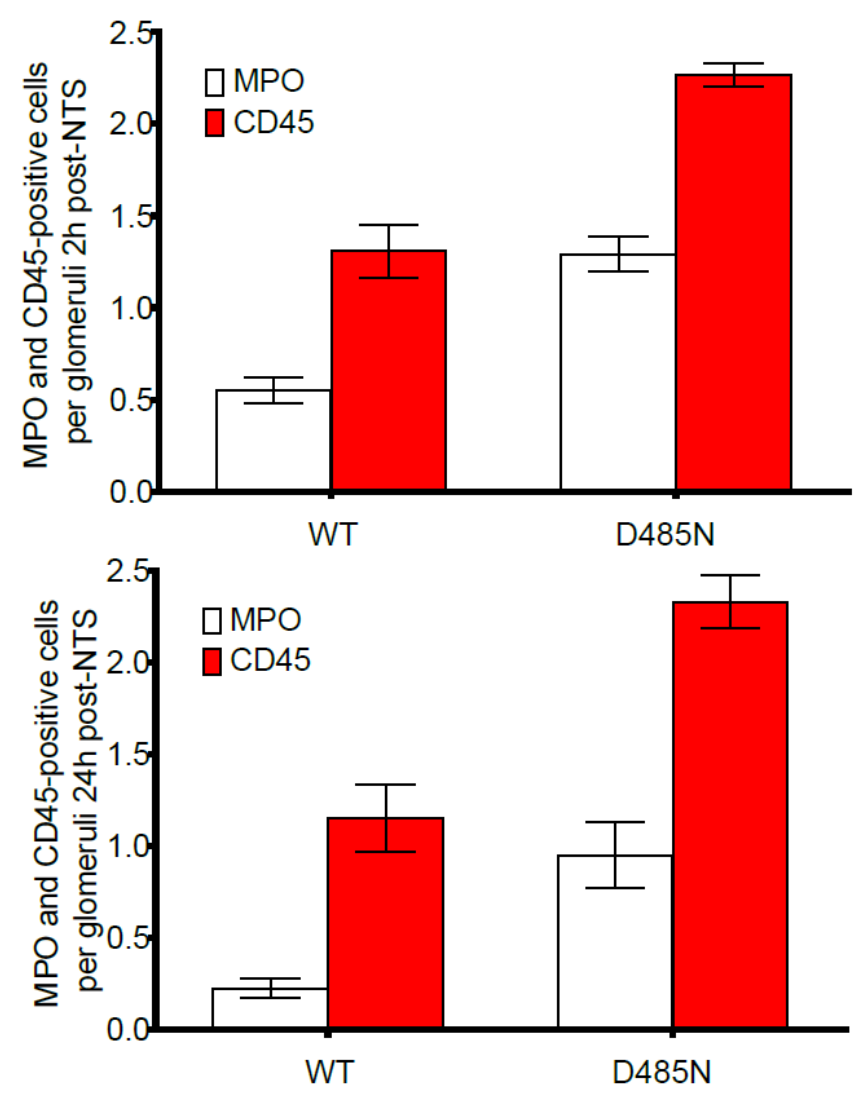

Figure 23 - CD45-positive and MPO-positive Cell Influx into Glomeruli

Immunohistochemistry was used to assess influx of general leukocytes $\left(\mathrm{CD} 45^{+}\right.$cells) and neutrophils with other minor staining ( $\mathrm{MPO}^{+}$cells) at both 2 hours and 24 hours post NTS administration. The result was increased CD45 and MPO staining at both time points for ABIN1[D485N] expressing mice. ( $\mathrm{n}=4-5$ per condition and 30 glomeruli per sample)

Korte, unpublished. 

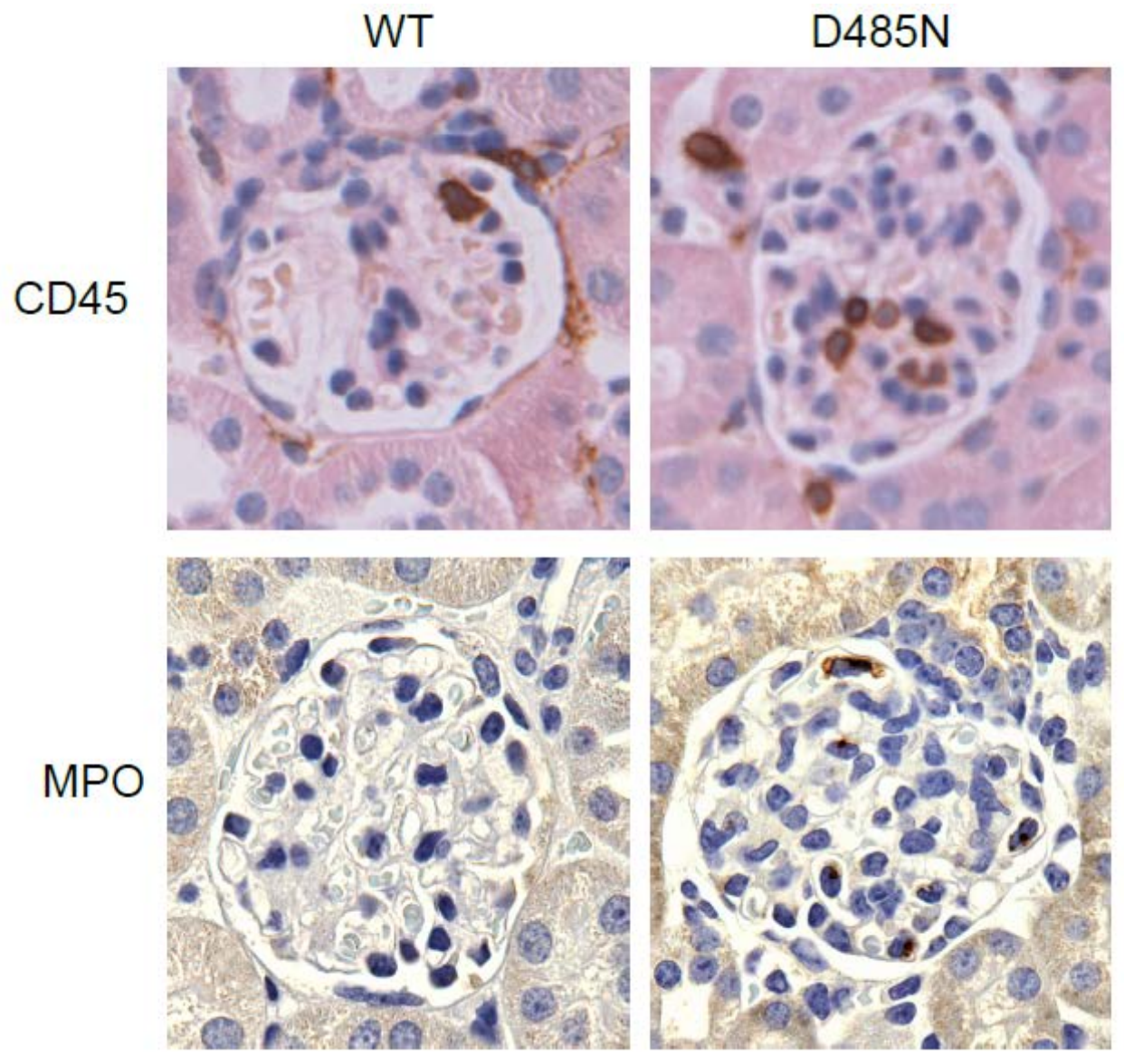

Figure 24 - Representative Images for Immune Cell Influx into Glomeruli Post-NTS

These representative images provide examples of what was described in Figure 23. 

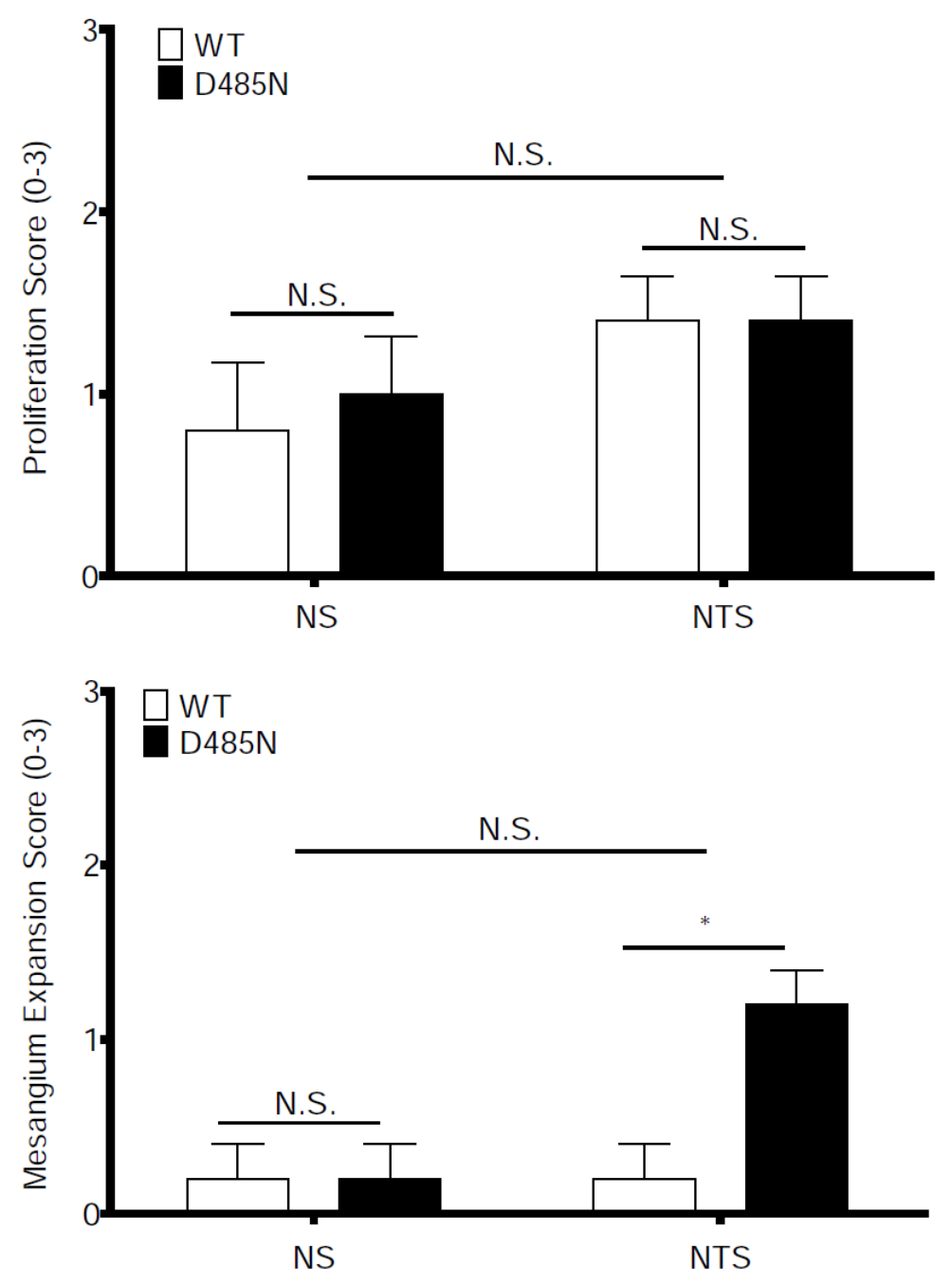

Figure 25 - Histological Analysis of PAS-stained Sections

Blinded analysis of PAS-stained sections of mouse kidney cortex 24 hours following administration of either NS or NTS found only minor differences. All conditions were statistically identical to ABIN1 WT animals given control serum (NS), with the exception of a slight elevation of matrix expansion in ABIN1[D485N] mice treated with NTS. ( $\mathrm{n}=$ 5 per condition with 30 glomeruli counted per sample.) Student's T-test compared values between two groups.

Korte, unpublished. 

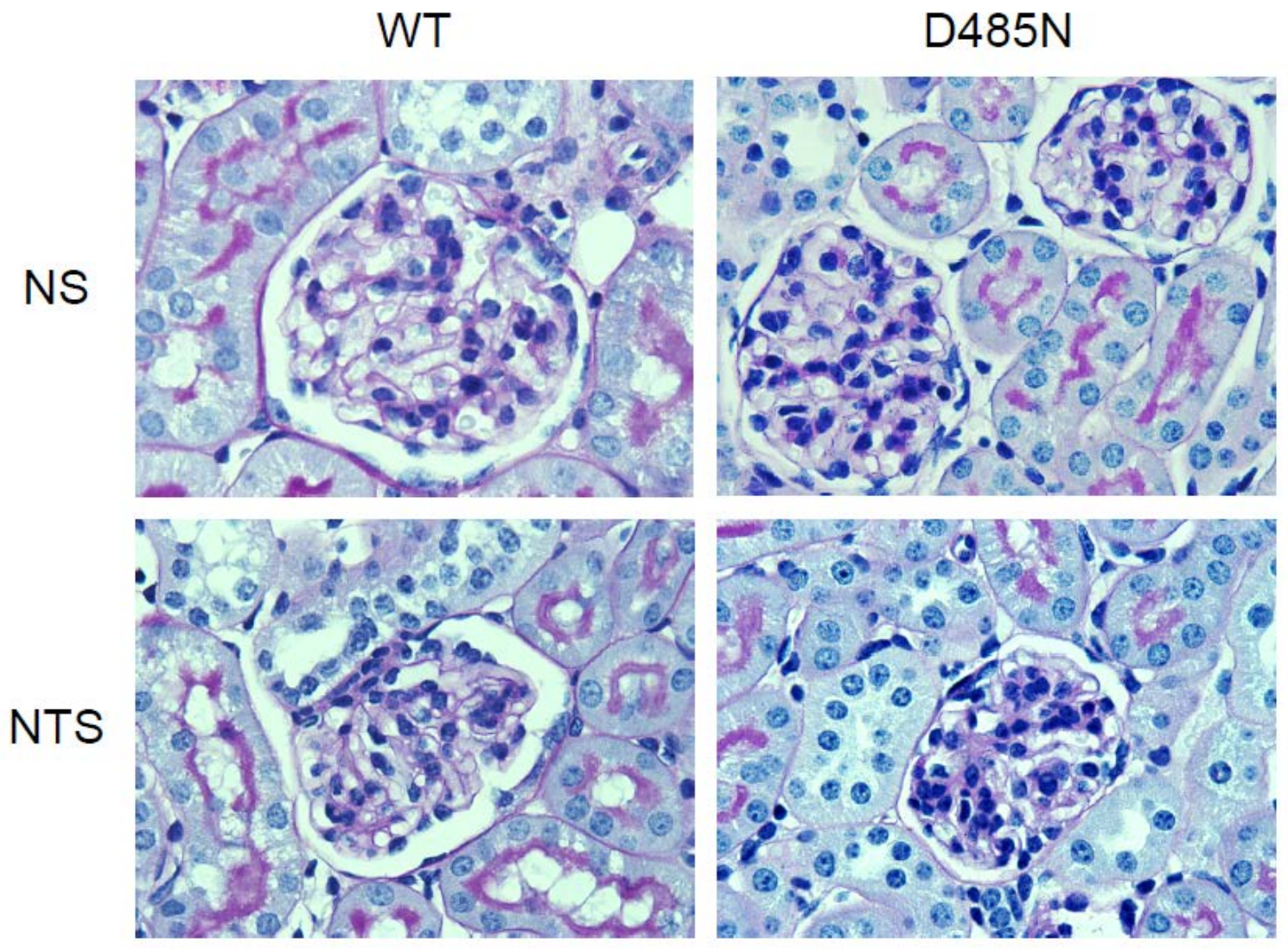

Figure 26 - Representative Images for Histological Analysis of Glomeruli Following NTS

These representative images provide examples of what was described in Figure 25. Korte, unpublished. 
a

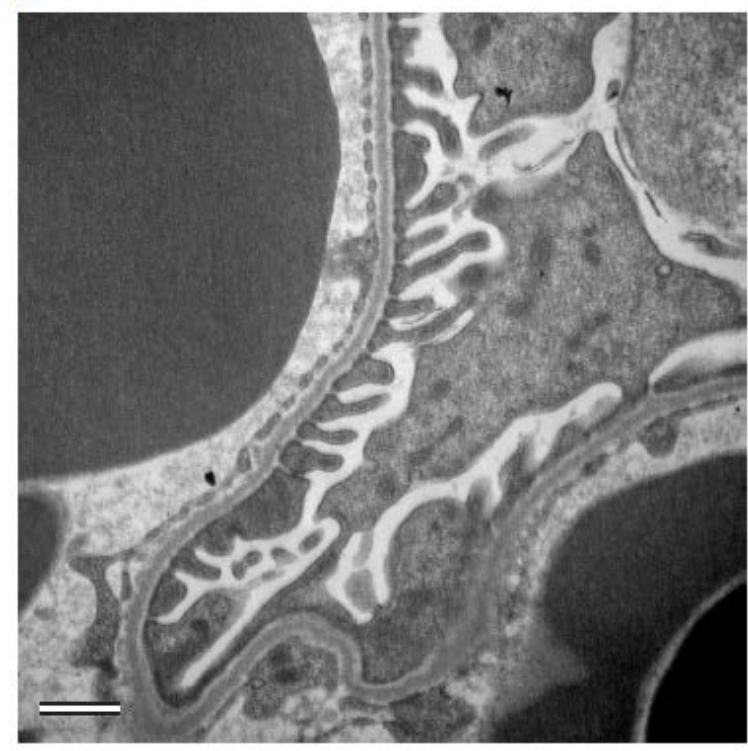

b

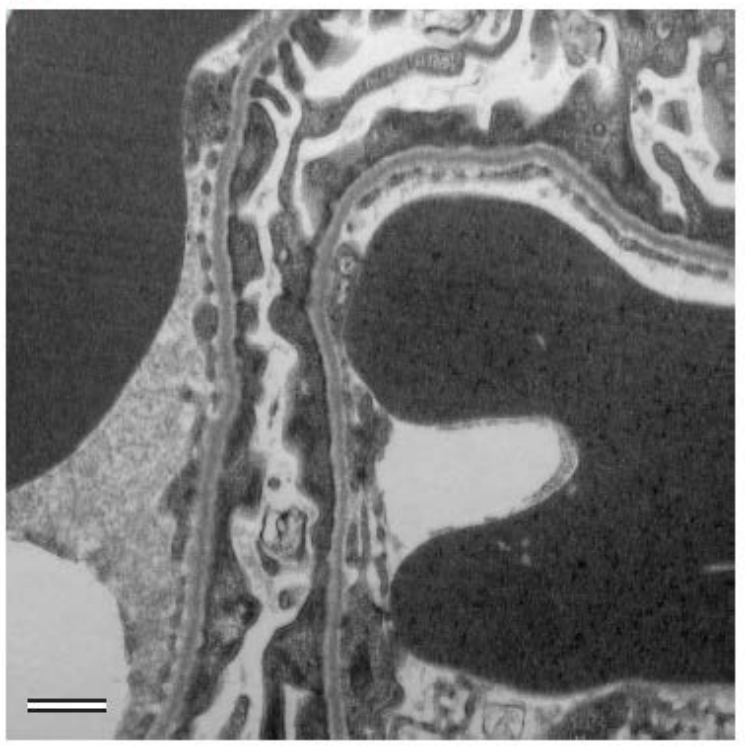

Figure 27 - Ultrastructural Analysis of NTS-induced Changes to Glomerular

\section{Basement Membrane}

Changes to the glomerular podocyte were noted 24 hours following NTS administration.

(a) TEM of ABIN1 WT glomeruli 24 hours post-NTS showed minor foot process

effacement. (b) ABIN1[D485N] mice show massive foot process effacement at 24 hours post-NTS. Representative images highlight this discrepancy. Endothelial cell changes and basement membrane thicken were not widespread. Scale bar represents 500nm. Korte, unpublished. 
corresponds to increased proteinuria and worsening renal function[187]. Figure 28 shows a significant reduction in WT-1 stained nuclei per glomerular section in ABIN1[D485N] mice, compared to WT, both at endogenous levels using normal sera as well as 24 hours following anti-GBM administration. Representative images are shown in Figure 29.

To determine production of mediators of inflammation by glomerular cells, glomeruli were purified from renal cortex by sieving, RNA was extracted, and expression levels of specific transcripts were determined using a custom qRT-PCR analysis. Genes included in this analysis were documented previously as contributing to GN either directly or as biomarkers and included adhesion molecules (VCAM1, ICAM1), profibrotic/proinflammatory mediators (TGF $\beta 1, \mathrm{C} 1 \mathrm{qB}, \mathrm{C} 3, \mathrm{CD} 74, \mathrm{EGF})$, interferons (IFN $\alpha 1$, IFN $\gamma)$, chemokines and cytokines (MCP-1, CCL5, CXCR7, TNF- $\alpha$, IL1 $\alpha$, IL12b, IL17a), cytoskeletal/matrix proteins (MYH9, MMP2, MMP7, TIMP2), or NF-кB

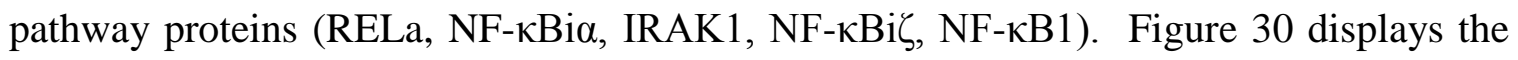
differences in glomerular gene expression between WT and ABIN1[D485N] mice 24 hours after NTS administration. mRNA expression of a number of proinflammatory cytokine and adhesion proteins was significantly increased in ABIN1[D485N] mouse glomeruli. Matrix metalloproteinase 7 (MMP7) exhibited a 40-fold increase in ABIN1[D485N] mouse glomeruli, compared to WT. Other notable anti-GBM-induced increases in ABIN1[D485N] mouse glomeruli included VCAM1 (3.00 fold), IL1 $\alpha$ (13.21 fold), IL17 $\alpha$ (2.14 fold), TNF- $\alpha$ (5.88 fold), CCL2/MCP-1 (1.97 fold) and EGF (1.98 fold). 


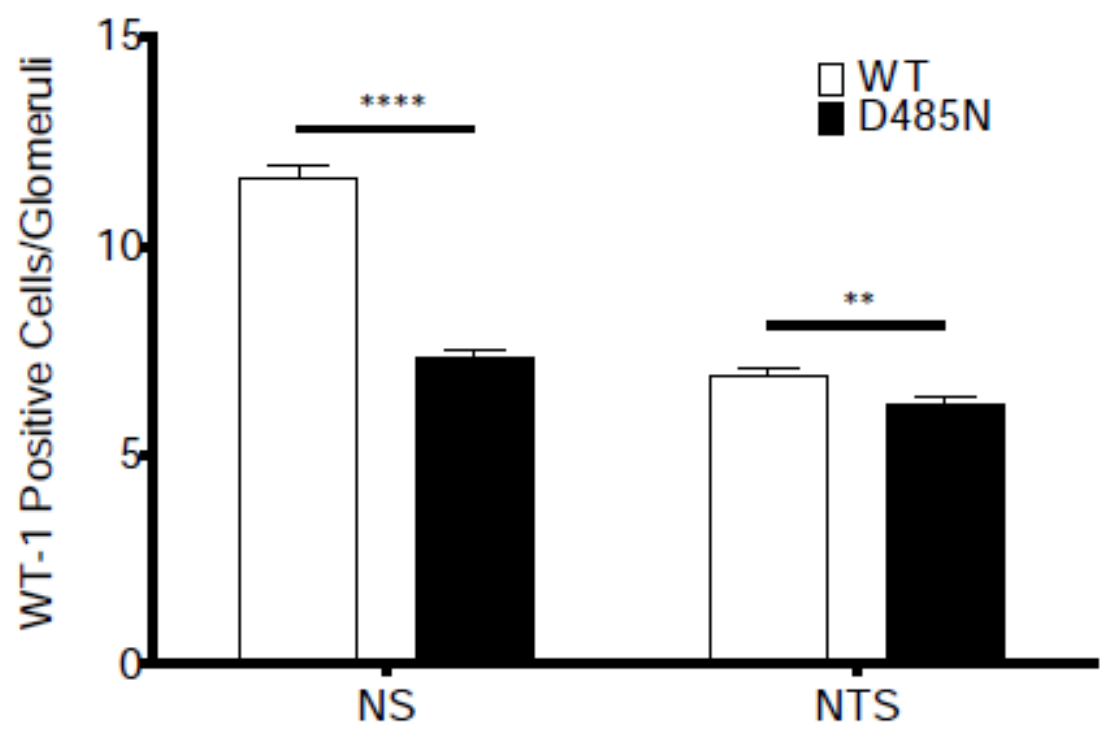

Figure 28 - WT1-staining per Glomeruli

Wilms Tumor 1 (WT1) is a nuclear marker of mature podocytes. Glomerular WT1 staining following control (NS) or NTS treated animals represents podocyte loss. ABIN1[D485N] mice exhibited a lower WT1 positive cell count per glomeruli than ABIN1 WT under either control or damaged conditions. $(n=4$ per condition, with 30 glomeruli counted for each sample) Bars represent SEM. T-test was used to compare values between two groups. $\left({ }^{* *} \mathrm{p}<0.01,{ }^{* * * *} \mathrm{p}<0.0001\right)$ 

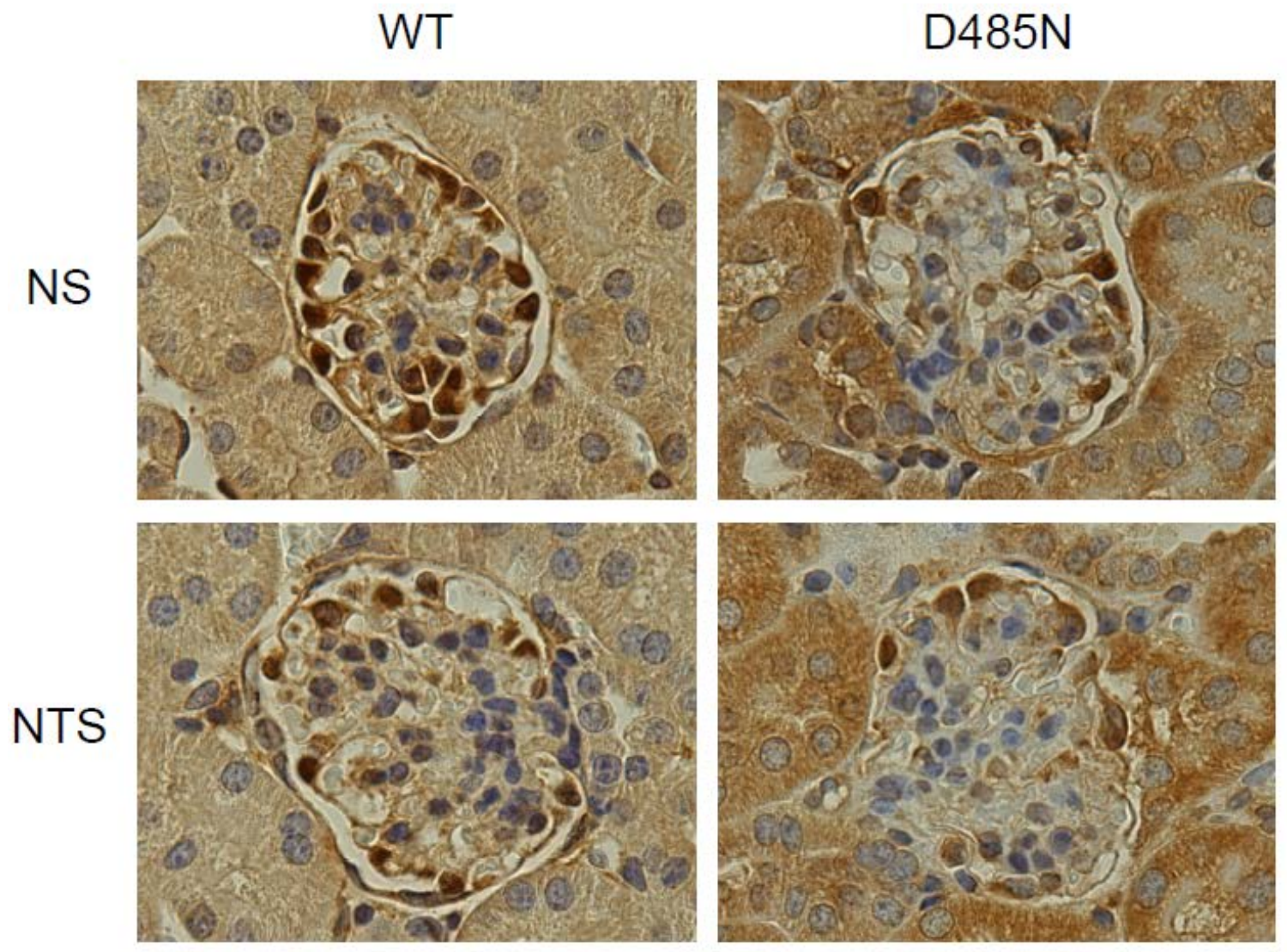

Figure 29 - Representative Images for WT1 Staining

These representative images provide examples of what was described in Figure 28. Korte, unpublished. 


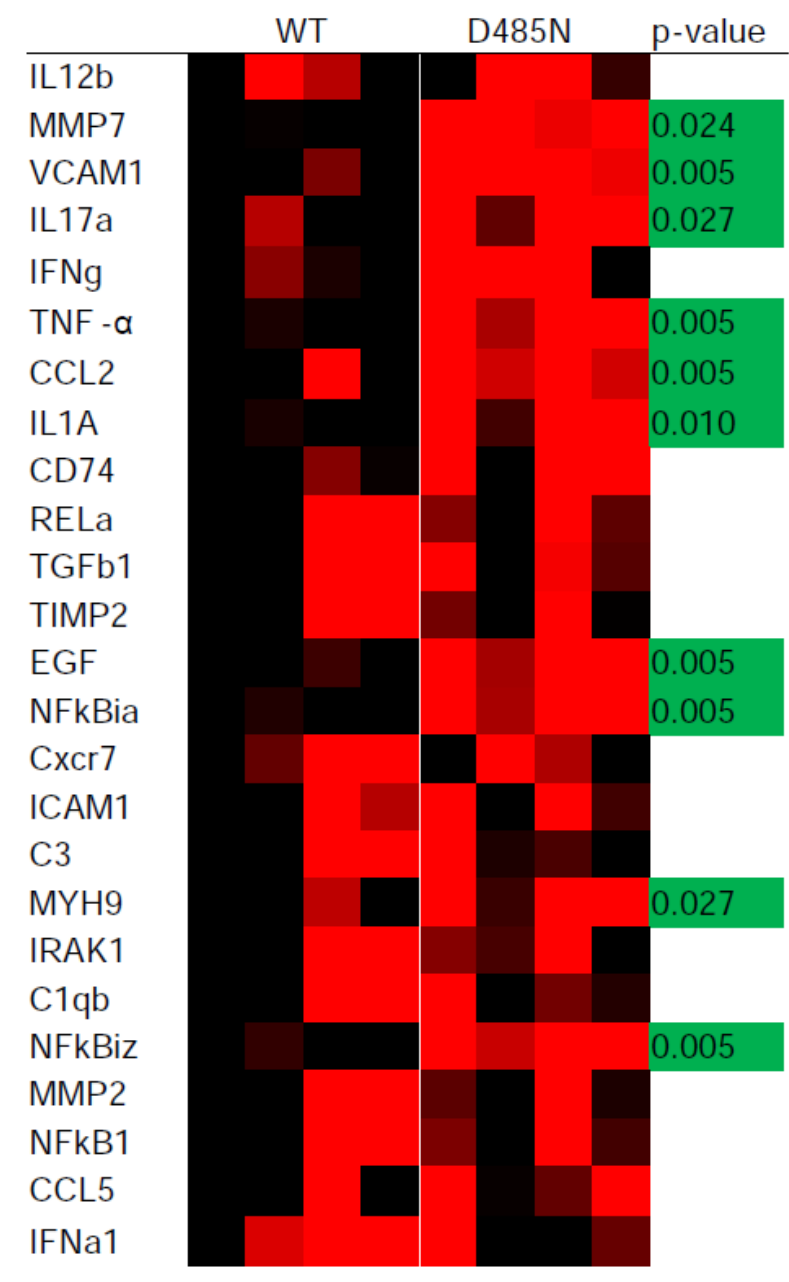

Glomerular Gene Expression

Figure 30 - Expression of Proinflammatory Markers from Isolated Glomeruli from WT and ABIN1[D485N] Mice

Isolated glomeruli from WT or ABIN1[D485N] kidneys showed marked transcriptional changes in a number of proinflammatory cytokines, chemokines and adhesion molecules. GAPDH served as a housekeeping gene and Student's T-test was used to compare between two groups. ( $\mathrm{n}=4$ per condition)

Korte, unpublished. 
Loss of ABIN1 ubiquitin binding function in podocytes results in excess production and secretion of NF- $\mathrm{K}$ targeted proinflammatory mediators.

Previous studies suggested NF- $\mathrm{BB}$ activation plays a role in development of GN

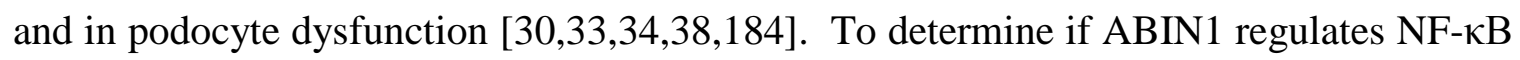
activation and proinflammatory responses in podocytes, changes in NF-кB-dependent gene expression were assessed in WT and ubiquitin binding null ABIN1[D472N] expressing podocytes following 3-hour stimulations with TNF- $\alpha$. The array assessed NF$\kappa \mathrm{B}$ target genes categorized as chemokines or cytokines (27), inflammatory markers (33), immune mediators (30). Figure 31 shows that proinflammatory NF- $\kappa \mathrm{B}$ target gene expression was upregulated in ABIN1[D472N] podocytes. Those findings were confirmed using single target RT-qPCR for notable genes involved in GN pathogenesis, including MCP-1 (CCL2), IL-8, CSF2 (GM-CSF), and CSF3 (G-CSF), and significant to at least $\mathrm{p}<0.01$ at every time point (Figure 32). Target gene expression in ABIN1[D472N] podocytes was found to be 4-10 fold higher for each target even without exogenous stimulation. To confirm that increased transcript results in increased protein secretion, podocyte supernatants were collected without contamination by FBS or exogenous TNF- $\alpha$, as outlined in Materials and Methods. ELISA for MCP-1, IL-8 and TNF- $\alpha$ confirmed that increased secretion of these NF- $\kappa$ B regulated proinflammatory proteins correlated with increased gene expression in ABIN1[D472N] podocytes (Figure 33).

Interaction between podocytes and neutrophils in proinflammatory activation and injury 


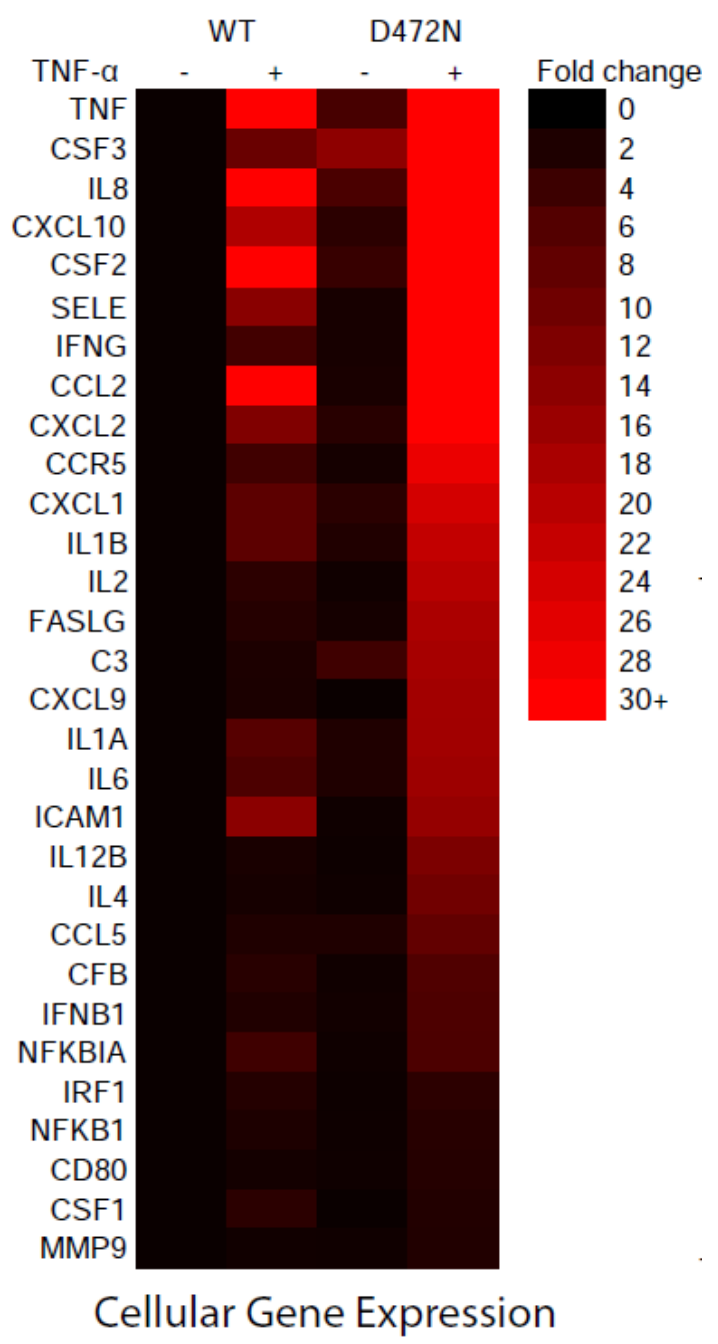

Figure 31 - Expression of Proinflammatory Factors in Cultured Podocytes

RT-qPCR array showed transcriptional changes similar to what was reported in isolated glomeruli following a 3-hour stimulation by TNF- $\alpha$. Notably, proinflammatory cytokines and chemokines were expressed at higher levels in ABIN1[D472N] expressing podocytes with or without stimulation. GAPDH served as a housekeeping gene and Student's Ttest was used to compare between two groups. ( $\mathrm{n}=2$ per condition)

Korte, unpublished. 

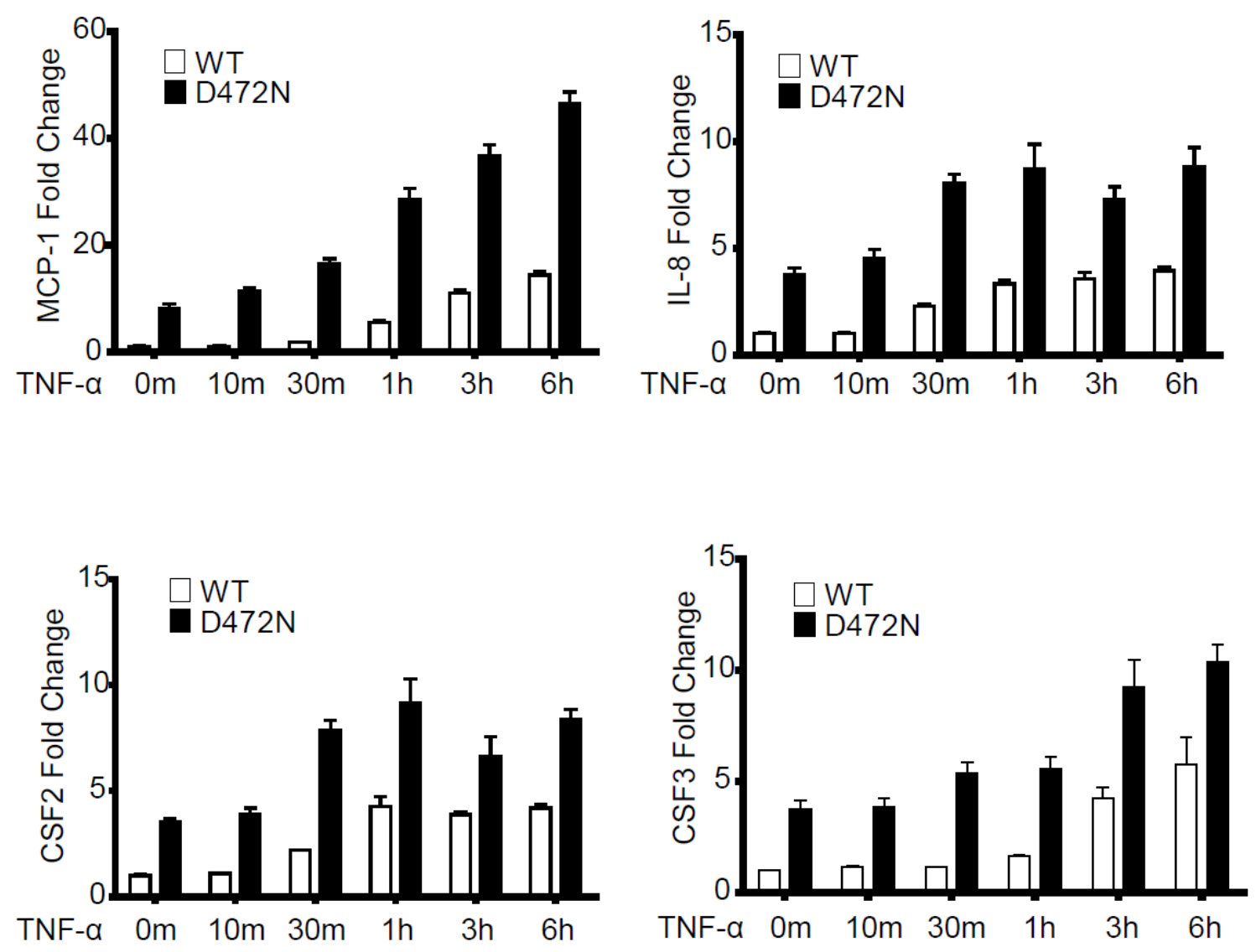

Figure 32 - Single Target RT-qPCR Validation of Array Data

Validation of four cytokines noted in the array showed significant increases in expression using single-target RT-qPCR with stimulation times ranging from 0-6 hours. Expression of these targets was shown to be 4-10 fold higher even at basal levels in cells expressing ABIN1[D472N]. GAPDH served as a housekeeping gene, all experiments were carried out in triplicate. ( $\mathrm{n}=3-5$ per condition) All points are significant by Student's T-test between WT and ABIN1[D472N] with $\mathrm{p}<0.01$.

Korte, unpublished. 

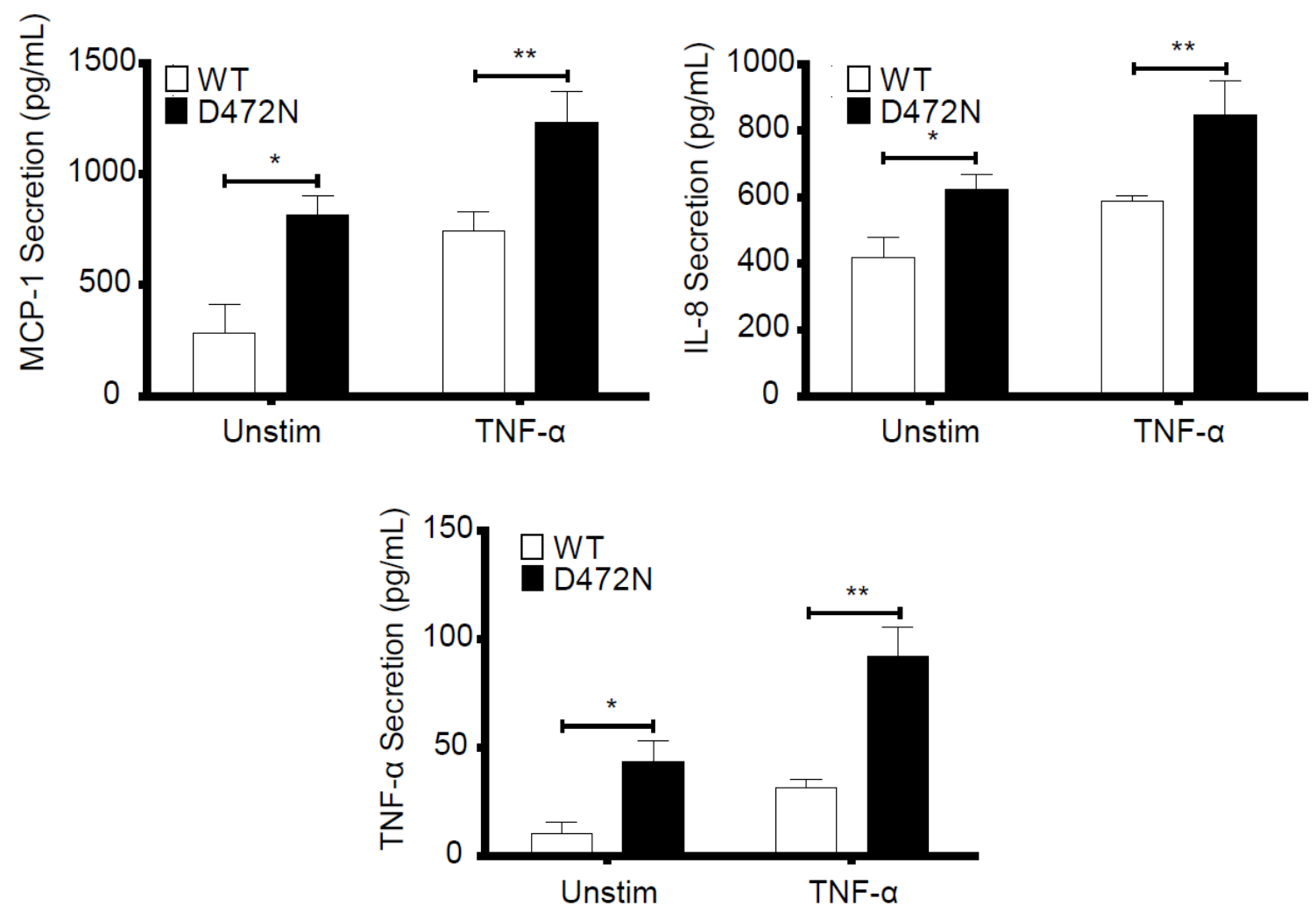

Figure 33 - ELISA Quantification of Secreted Cytokines from Cultured Podocytes

Quantification of secreted proteins by ELISA showed the differences in transcript corresponded to increased secretion of cytokines and chemokines into the cell culture supernatant. All experiments were completed in triplicate and repeated at least 3 times. Student's T-test compared values between two groups. $\left({ }^{*} \mathrm{p}<0.05,{ }^{* *} \mathrm{p}<0.01\right)$ Korte, unpublished. 
Figure 23 and Figure 24 show that glomerular leukocyte accumulation is enhanced in vivo in ABIN1[D485N] expressing mice following NTN. To determine if ABIN1 dysfunction in podocytes was a potential mechanism for enhanced leukocyte recruitment into the glomerulus, the ability of WT and ABIN1[D472N] podocyte supernatants on chemotaxis of primary human neutrophils was tested (Figure 34). Podocytes expressing WT ABIN1 produced a secretome capable of recruiting cells into a synthetic membrane when unstimulated, and secretome from WT cells previously stimulated with TNF- $\alpha$ increased this effect. ABIN1[D472N] expressing podocytes produced a secretome with stronger chemotactic capabilities than ABIN1 WT expressing cells at both basal levels and when stimulated. The ability of podocyte supernatants to stimulate neutrophil degranulation in primary human neutrophils was examined by measuring plasma membrane CD35 (secretory vesicle marker) and CD66b (specific granule marker) expression (Figure 35). Although podocyte supernatants following incubation with TNF- $\alpha$ induced a significant increase in CD35 and CD66b expression, there were no differences in degranulation induced by supernatants from WT and ABIN1[D472N] podocytes.

To determine if the increased chemoattraction and degranulation induced by podocyte products affected podocyte integrity, the effect of neutrophil granule contents on podocyte cytoskeletal morphology was assessed. Podocytes were cultured for $24 \mathrm{hr}$ with supernatants from primary human neutrophils stimulated without (control) or with TNF- $\alpha / \mathrm{fMLF}$ for 30 minutes, and actin stress fibers were visualized using rhodaminephalloidin staining. Both WT and ABIN1[D472N] podocytes displayed typical healthy stress fiber structure following 24 hour incubation with control neutrophil supernatant, 


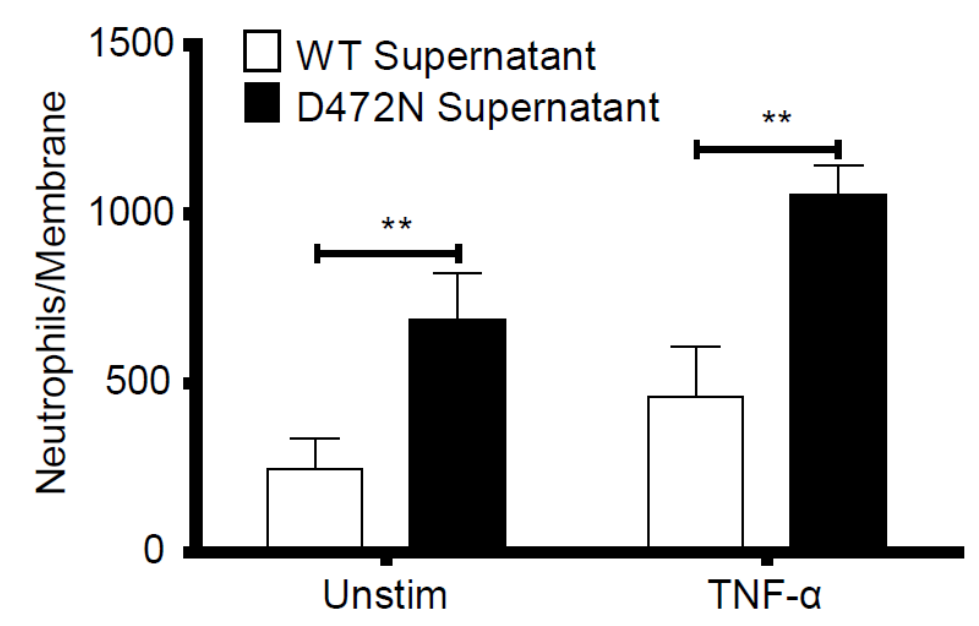

Figure 34 - Induction of Neutrophil Chemotaxis by Podocyte Cell Culture Supernatant

Chemotaxis of primary human donor neutrophils into synthetic membranes was induced by podocyte cell culture supernatant over control cell culture media, and stimulation of the podocytes before washing and collection of their media resulted in greater rates of chemotaxis. Furthermore, podocytes expressing ABIN1[D472N] were able to induce twice the rates of chemotaxis as ABIN1 WT. $(\mathrm{n}=3$ distinct cell culture supernatants per condition, each used to stimulated 3 different donors of primary neutrophils.) Student's T-test compares values between groups. $\left({ }^{* *} \mathrm{p}<0.01\right)$

Korte, unpublished. 

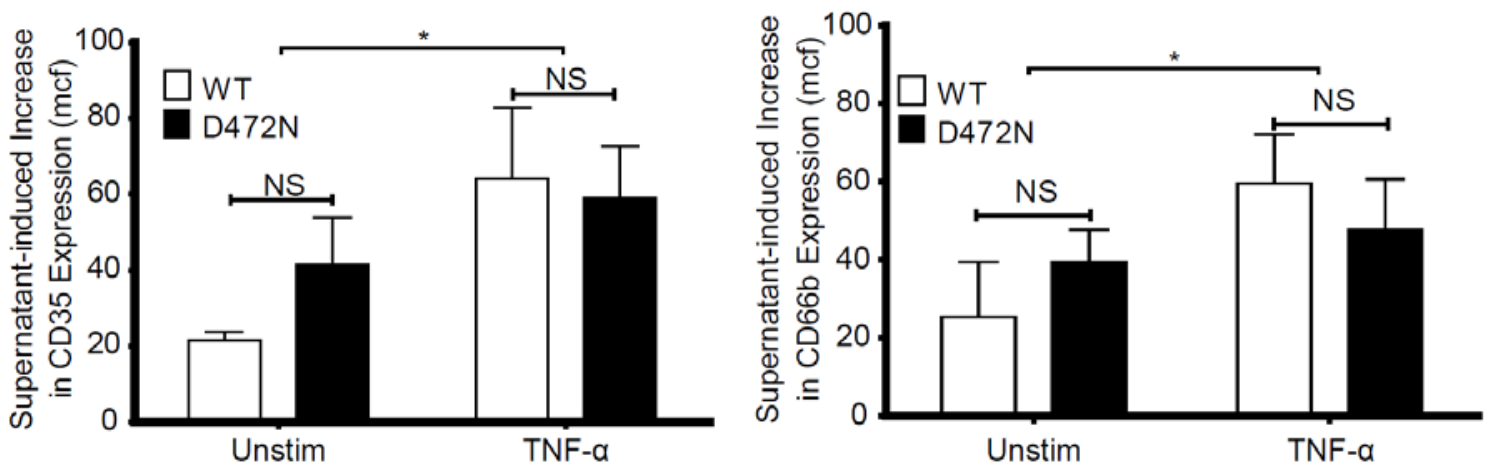

Figure 35 - Induction of Neutrophil Degranulation Markers by Podocyte Cell

\section{Culture Supernatant}

Podocyte cell culture supernatant was able to induce expression of degranulation markers, C35 and CD66b, on human primary neutrophils. No significant differences were noted between ABIN1 WT and ABIN1[D472N] podocyte supernatant, although podocytes stimulated before washing and collection of supernatant were able to induce significant expression of degranulation markers on primary neutrophils. Expression of CD35 and CD66b was quantified by flow cytometry. ( $\mathrm{n}=3$ distinct cell culture supernatants per condition, each used to stimulated 3 different donors of primary neutrophils.) Student's T-test compares values between groups. $\left({ }^{*} \mathrm{p}<0.01\right)$

Korte, unpublished. 
including long stress fibers extending at least half the length of the cell and crossing the nucleus, with a primarily unidirectional distribution (Figure 36). Anisotropy is a measurement of the angularity of the f-actin stress fibers which uses rotational statistics to quantify changes to f-actin organization using FibrilTool which utilizes raw images and avoids potential bias of reprocessing [84]. Figure 37 shows the anisotropy of WT and ABIN1[D472N] podocyte stress fibers. Anisotrophy was significantly reduced in ABIN1[D472N] podocytes, compared to WT. TNF- $\alpha /$ fMLF stimulated neutrophil supernatant caused a significant loss of stress fiber anisotropy compared to control supernatant in both WT and ABIN1[D472N] podocytes. Each cell assessed by FibrilTool was also examined for three additional features of podocyte morphologic alteration; transnuclear stress fiber loss, ring formation, and actin-rich center (ARC) formation (Figure 38). All three of these features were found to be significantly increased in ABIN1[D472] podocytes exposed to stimulated neutrophil granules, compared to WT. Multivariate logistical regression analysis produced odds ratios of 7.00, 3.87, and 3.85 for transnuclear fiber loss, ring formation, and actin rich center (ARC) formation, respectively, in ABIN1[D472] podocytes (Figure 39).

\section{Inhibition of neutrophil degranulation reduces proteinuria in vivo}

The altered podocyte cytoskeleton induced by supernatants from stimulated neutrophils and the ability of supernatants from stimulated podocytes to induce neutrophil degranulation suggested that release of granule neutrophil contents participates in podocyte injury leading to proteinuria in NTN. To test this hypothesis, we utilized a 


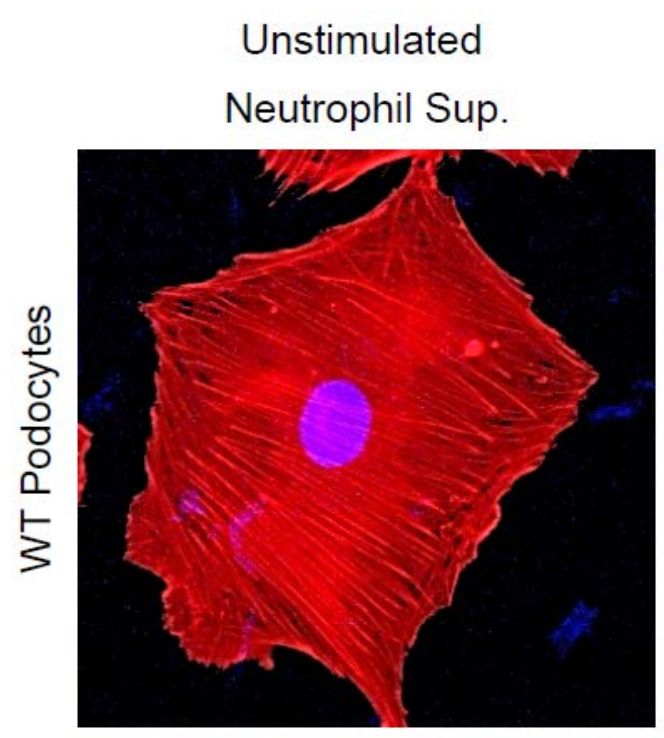

TNF-a-fMLF Stimulated

Neutrophil Sup.
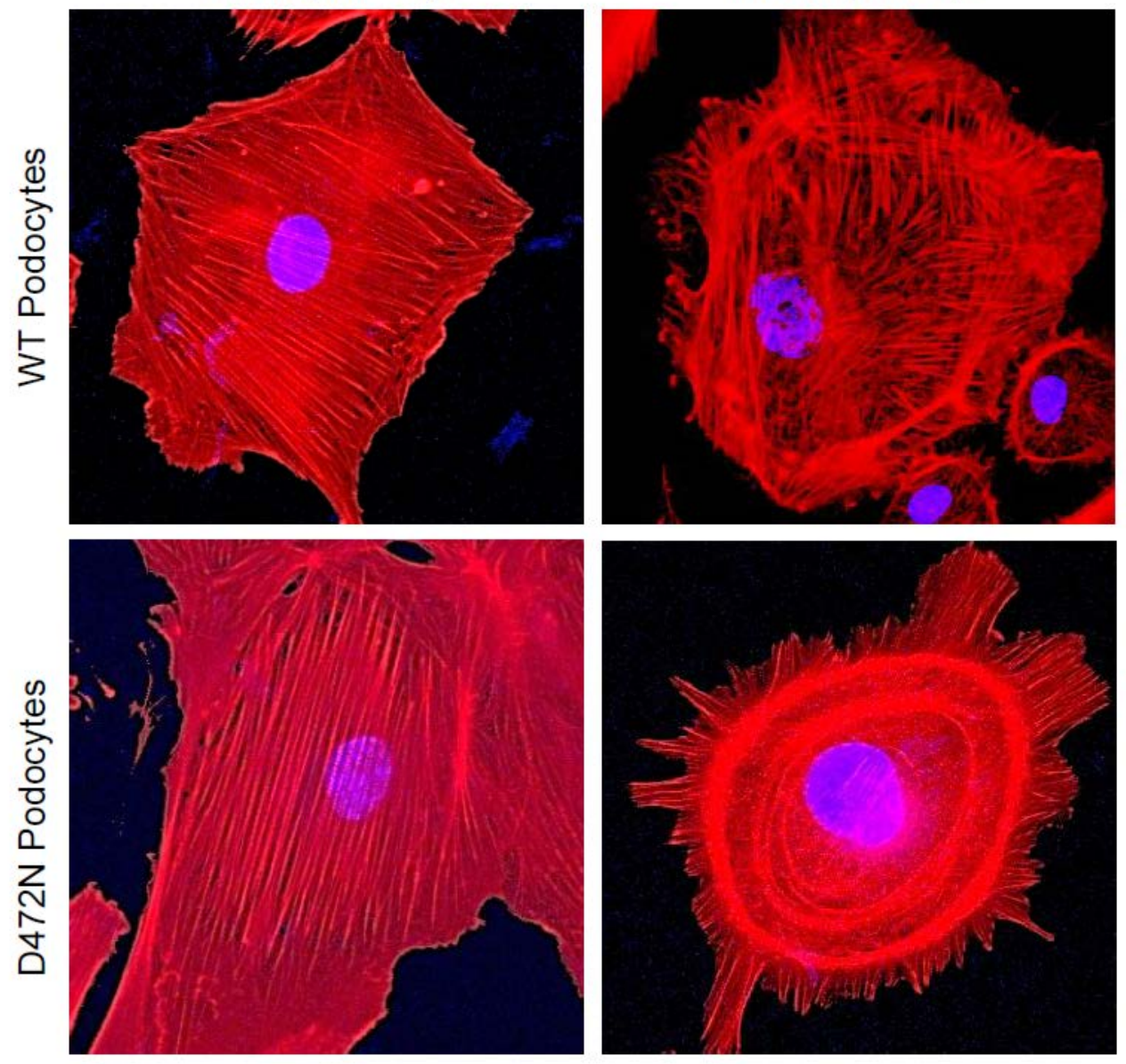

Figure 36 - Neutrophil Granules Induce Cytoskeletal Remodeling in Cultured

\section{Podocytes}

Neutrophil granules were shown to induce major cytoskeletal remodeling of cultured podocytes. Representative images of rhodamine-phalloidin stained f-actin show parallel stress fibers in podocytes exposed to supernatant from unstimulated neutrophils, but loss of both length and orientation of these stress fibers upon 24 hour exposure to supernatant from TNF- $\alpha$-fMLF stimulated neutrophils.

Korte, unpublished. 


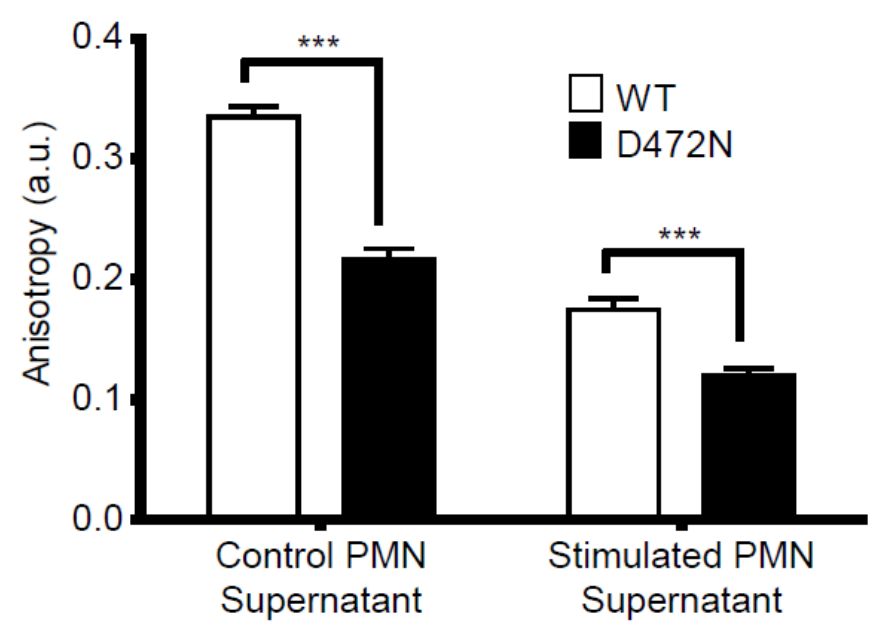

Figure 37 - Anisotropy of Podocyte F-actin Following Exposure to Neutrophil

\section{Granules}

The loss of stress fiber orientation is quantified using Fibriltool as described in the Chapter 2. The anisotropy of f-actin was significantly reduced by exposure to neutrophil $(\mathrm{PMN})$ granules and also in podocytes expressing ABIN1[D472N] ( $\mathrm{n}=3$ samples per condition with at least 30 cells analyzed per sample).

Korte, unpublished. 


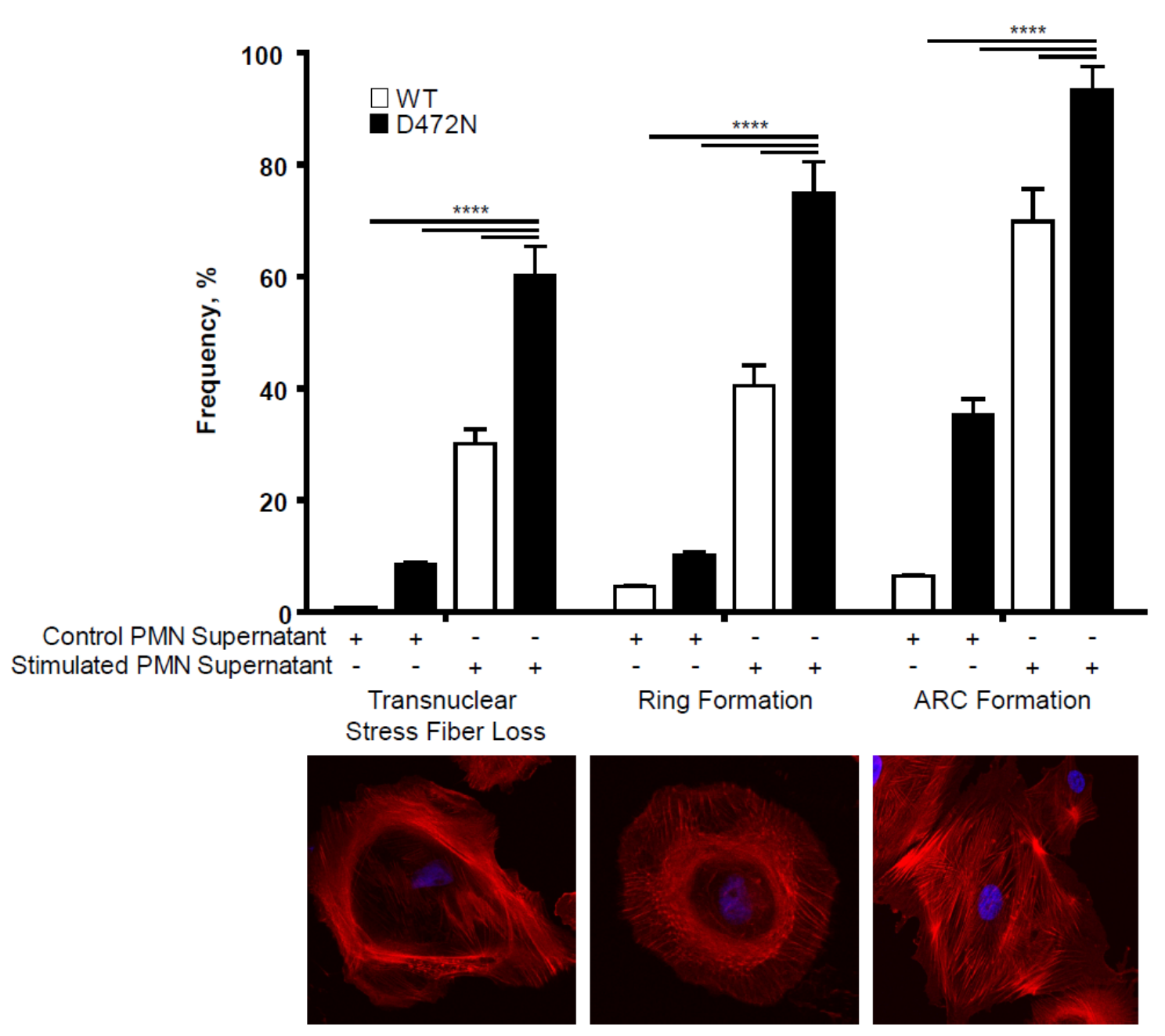

Figure 38 - Assessment of Common Morphogenic Pathologic Changes in Podocyte

\section{Cytoskeleton}

Other commonly cited morphological changes assessed were loss of transnuclear stress fibers, ring formation and ACR formation. Representative images for each are given. All experiments were completed in triplicate with at least 3 separate experiments performed. Multivariate logistic regression analysis was used to compare dichotomous outcomes of actin remodeling and accounted for both ABIN1 genotype and exposure to granules.

Korte, unpublished. 


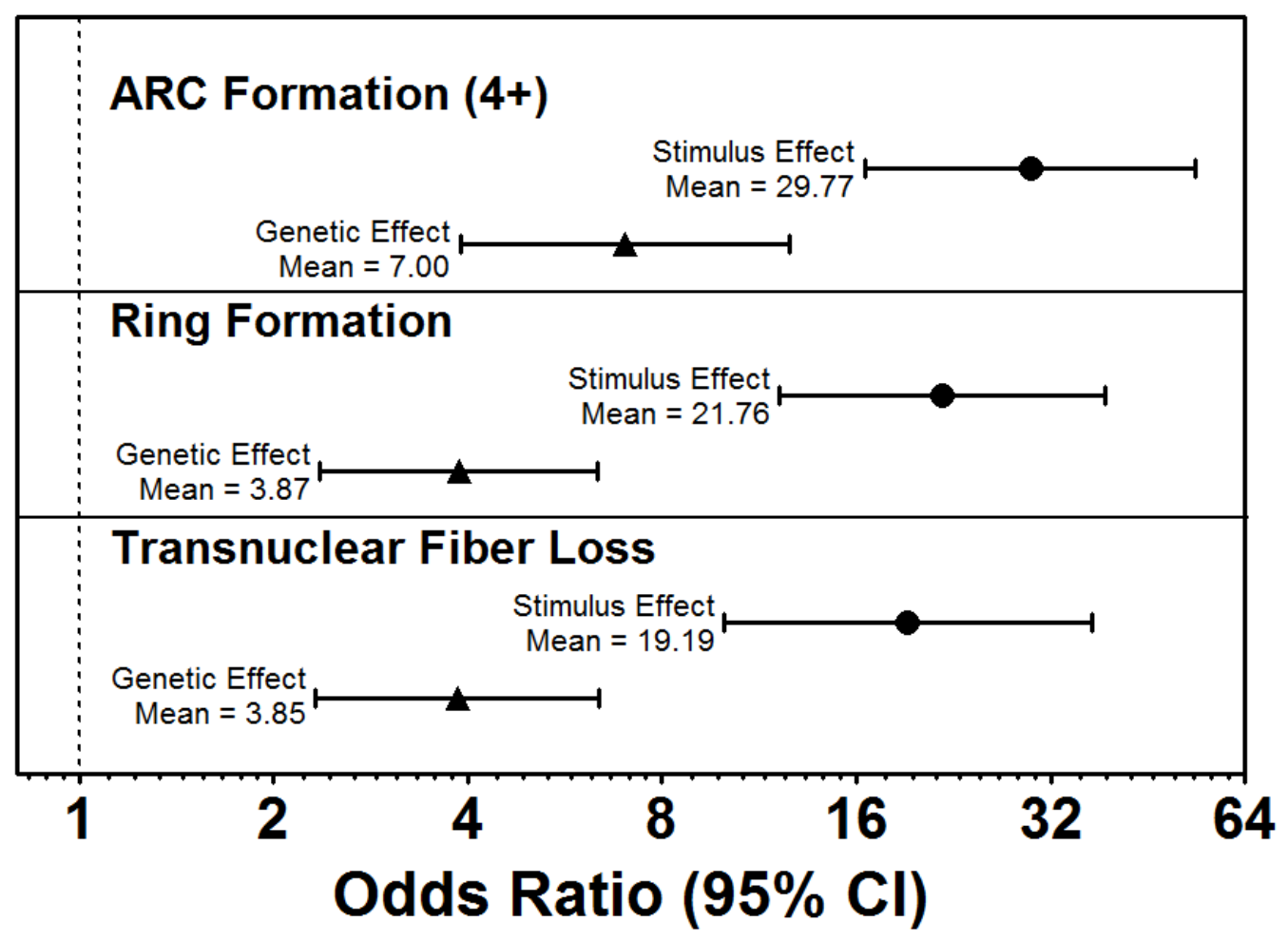

Figure 39 - Odds Ratio of Genetic Effect and Neutrophil Granules on Pathologic Podocyte Cytoskeletal Rearrangements

To properly separate the dichotomous outcomes resulting from the stimulus (neutrophil granules) versus the ABIN1 genotype, multivariate logistic regression analysis was used to odds ratios and clearly showed an impact of each on podocyte actin remodeling. Korte, unpublished. 
unique TAT-SNAP-23 fusion protein to inhibit neutrophil degranulation in vivo $[89,188-$ 190]. Both WT and ABIN1[D472N] mice treated with TAT-SNAP-23 at the time of anti-GBM administration and 6 hours later showed a significant reduction in proteinuria at 24 hours (Figure 40a). Although the absolute levels of proteinuria were higher in both groups of ABIN1[D472N] mice, compared to WT, the fold reduction in proteinuria as a response to TAT-SNAP-23 treatment was significantly lower in ABIN1[D485N] mice compared to WT (Figure 40b).

\section{$\underline{5.3-\text { Discussion }}$}

In the past few years, ABIN1 has emerged as a novel physiological inhibitor of NF- $\kappa \mathrm{B}$ and a genetic factor in autoimmunity and glomerulonephritis (GN), but a mechanistic role for ABIN1 function in GN had not been characterized [53,66,184]. In this study, we show that transgenic disruption of ABIN1 polyubiquitin binding activity (by mouse D485N and homologous human D472N mutation) results in enhanced podocyte injury and proinflammatory neutrophil activation following administration of nephrotoxic sera (NTS) containing antibodies directed against glomerular basement membrane (GBM) in mice and proinflammatory activation of cultured human-derived podocytes. The current paradigm is that GN is initiated from glomerular immune complex deposition, followed by proinflammatory activation by podocytes and other glomerular cells, however events regulating intrinsic kidney effects are comparatively understudied and unclearly defined [15,191]. Previous reports from our group and others 

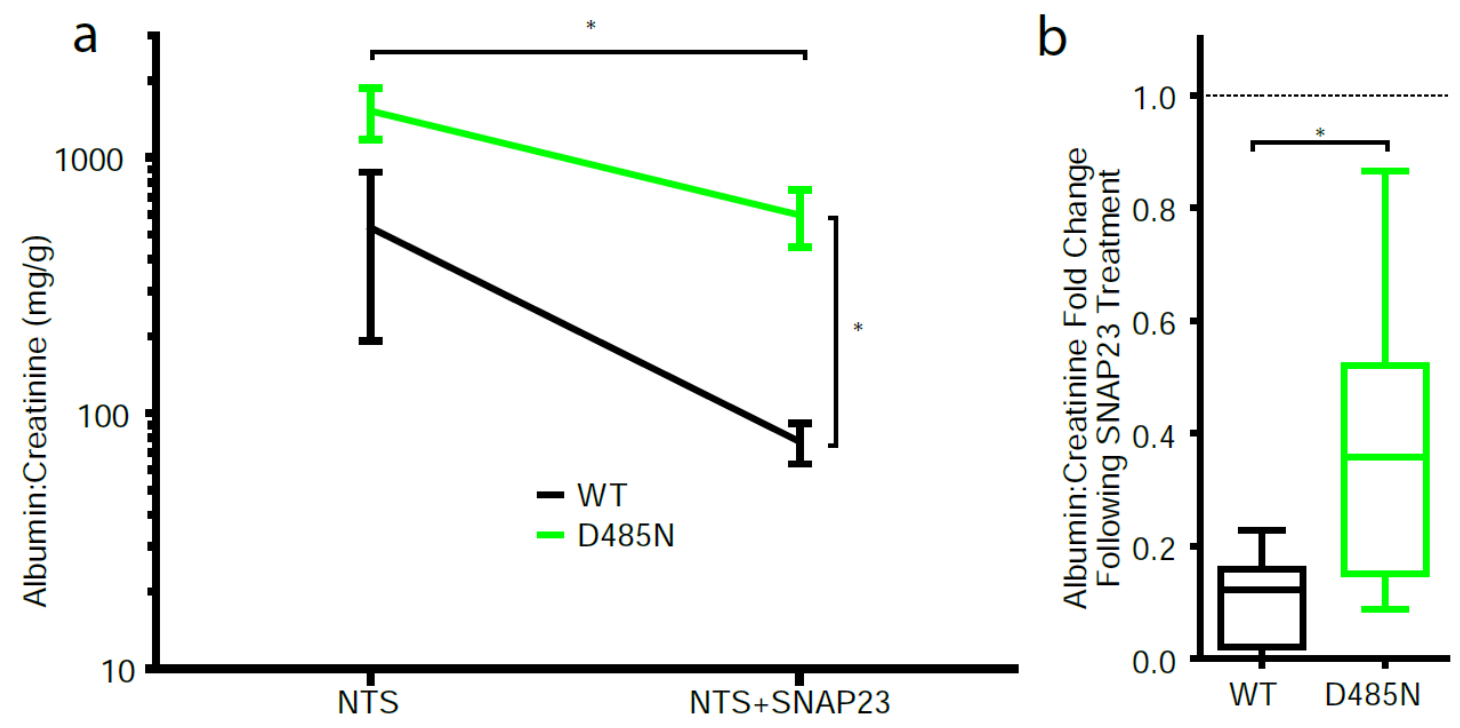

Figure 40 - TAT-SNAP23-mediated Inhibition of Neutrophil Degranulation Reduces Proteinuria

Inhibition of neutrophil degranulation in vivo reduces proteinuria due to NTS. (a) Administration of TAT-SNAP23 at 0 hours and 6 hours following administration of NTS markedly reduces proteinuria in ABIN1 WT expressing animals, but this reduction is muted in mutant animals. ( $n=6-9$ per condition) (b) Fold change reduction in average UAC following TAT-SNAP23 treatment was higher in WT mice than in ABIN1[D485N] mice. Student’s T-test compared values between two groups.

Korte, unpublished. 
indicate that immune deposition is mediated by autoantibody reactivity to endogenous and planted target glomerular antigens [5,192-195]. While it is presumed that these autoantibodies are nephritogenic, to date no pathogenic mechanisms have been defined. The NTS in vivo model was selected for our study for three reasons: (1) reports show glomerular NF-кB activation, (2) it shares the etiology of an important type of human GN without the requirement of presensitization of the immune system, and (3) rapid or acute development of proteinuria in response to NTS injection may allow investigation of the instigating pathogenic mechanism of podocyte injury [38,185,196]. Consistent with a podocyte injury postulate, we report no remarkable mesangial cell alterations, including a lack of mesangial cell expansion post NTS injection for both WT and ABIN1[D485N] mice and only minimal matrix expansion. There was, however, focal podocyte foot process effacement in WT kidneys on electron microscopy which was more pronounced in ABIN1[D485N] mice. Loss of glomerular WT-1 staining further supported podocyte dysfunction in $\alpha$-GBM-mediated GN. Urinary protein loss was more severe in ABIN1[D485N] mice following administration of $\alpha$-GBM. Proteinuria which was 9-fold higher in ABIN1[D485N] mice compared to WT and there was noted enrichment of $\mathrm{CD}^{+} 5^{+}$cells (leukocytes) and MPO staining (neutrophil azurophilic granules) in glomeruli of NTS treated ABIN1[D485N] mice compared to WT at both 2 hours and 24 hours following NTS administration. Interestingly, neutrophils were retained in the glomeruli at 24 hours to a much greater degree in ABIN1[D485N] mice than in WT which suggests either the immune system itself was mounting a stronger reaction to the NTS or something within the local tissue was acting on resident neutrophils and other immune cells to maintain their presence within the glomerulus. To explore the question 
of increased immune activation vs local tissue effects, bone marrow transplant produced chimeric mice and showed no effect of immune system genotype on the severity of GN resulting from NTS and instead suggested that the local genotype was indeed the driving force behind increased severity of renal dysfunction in ABIN1[D485N] mice. Differences between reactivity between 6-week old mice and 12-week old mice post bone marrow transplant were as expected and the well-described response of C57BL/6 mice to NTS allowed controlled glomerular damage that was neither the extreme nor the subdued categories of responses [197,198]. These findings indicated that glomerular loss of ABIN1 polyubiquitin binding function promotes autoantibody-mediated injury and proteinuria and suggests podocytes may be a cause rather than an effect of these pathogenic changes. The potential mechanism for $\alpha$-GBM-induced injury likely involves both immune and intrinsic renal tissues, but the mechanism behind their interaction is not well understood. Thus we sought to describe the functional mechanism for $\alpha$-GBMmediated injury using complementary in vivo, in vitro, and ex vivo techniques.

Glomerular immune cell infiltration and inflammation described above are hallmarks of GN. Going back to the 1990s, studies have shown correlation of elevated glomerular expression of NF-кB-regulated proinflammatory mediators MCP-1, TNF- $\alpha$, and IL-1 $\alpha$ with progression of GN in rodents and humans $[199,200]$. Renal cortex has previously been used to investigate renal expression changes of proinflammatory genes, but cortex samples will contain $70-90 \%$ tubule cells and their associated mRNA transcript, which would dampen early changes in a glomerulocentric injury like $\alpha$-GBMinduced nephritis. Thus, we performed RT-PCR analysis for NF-кB target and GNassociated genes using isolated glomeruli and found a significant increase in expression 
of many proinflammatory and chemotactic transcripts, including TNF- $\alpha$, MCP-1(CCL2), and IL-1 $\alpha$ in ABIN1[D485N] mice 24 hours after NTS injury. TNF- $\alpha$ is commonly overexpressed in both serum and urine of GN patients and correlates to disease severity, specifically in immune-complex GN, and podocytes can both produce and respond to TNF- $\alpha$ through the NF-kB pathway [201-206]. Thus, TNF- $\alpha$ was utilized as a stimulant to study inflammation and podocyte function relating to GN pathogenesis and ABIN1 function in vitro using human-derived cultured podocytes. Mirroring the in vivo findings, podocytes expressing the homologous human ABIN1 ubiquitin binding mutation (D472N) exhibit exacerbated NF-кB activation, increased MCP-1, CSF 1/2, and IL-8 mRNA expression, and amplified secretion of MCP-1, IL-8, and TNF- $\alpha$.

The glomerulus has been shown to be unique among capillary beds in its ability to recruit and retain leukocytes, allowing intrinsic renal cells a unique opportunity for intimate interaction with immune cells, specifically neutrophils [28]. An intrarenal role for neutrophils in the pathogenesis of human GN is implicated by their glomerular accumulation in kidney biopsies from patients with various type of GN (including MPGN, ANCA, LN and $\alpha$-GBM disease) and inversely by reduction in disease phenotypes with neutrophil depletion in various animal models of GN [3,207,208]. However, mechanisms for glomerular recruitment and retention of neutrophils and neutrophil-directed injury causing proteinuria in GN have not been resolved. The secretome of ABIN1[D472N] expressing podocytes amplified primary human neutrophil chemotaxis into synthetic membranes when compared to WT at both unstimulated levels and when the podocytes had been previously stimulated with TNF- $\alpha$. Furthermore, we found that podocytes possess the ability to induce neutrophil release of specific granules 
and secretory vesicles. These granules contain bactericidal proteins that also cause inflammatory tissue injury [209,210]. Although ABIN1[D472N] supernatant tended to produce higher levels of degranulation markers in neutrophils than WT at endogenous levels, this failed to achieve statistical significance. It was, however, clearly shown that stimulated podocytes of either genotype are able to produce a secretome capable of degranulating primary neutrophils. Thus, podocytes have the ability to both recruit neutrophils to the glomerulus and subsequently activate their release of injurious granule contents. To further explore this mechanism in vivo, we utilized an inhibitor of neutrophil degranulation, the chimeric protein TAT-SNAP23, which competitively binds complexed vesicular fusion proteins, vesicle-associated membrane protein-2 and syntaxin-4, to block release of granules from neutrophil cytosol $[188,189]$. Inhibition of neutrophil degranulation by TAT-SNAP23 resulted in marked decreases in $\alpha$-GBM-mediated proteinurea in both WT and ABIN1[D485N] expressing mice. It was interesting to note that TAT-SNAP23 pretreatment of WT mice resulted in a 9.5-fold reduction in UAC while ABIN1[D485N] mice only exhibited a 2.6 fold reduction. This is further evidence that the genotype of the intrinsic renal cells, specifically podocytes, is an underappreciated pathogenic factor in glomerular maintenance and protection from immune-mediated damage.

The interaction between podocytes and neutrophils is not one-sided and proper ABIN1 function within podocytes was shown to be of great importance for limiting pathogenic podocyte responses following an insult. Actin regulation is paramount in slit diaphragm maintenance and renal function, and disorganization of actin stress fibers is a classic sign of podocyte damage [211-215]. Neutrophil granules clearly induce 
remodeling of cultured podocyte actin fibers shown by a reduction in the anisotropy of podocyte actin fibers, as ABIN1[D472N] expressing podocytes exhibited stress fibers which were significantly less organized than WT when subjected to either control neutrophil supernatant (no or few granules) or neutrophil supernatant fully degranulated by 30 minute exposure to TNF- $\alpha$ fMLF (high granule concentration). Furthermore, additional metrics of podocyte cytoskeletal rearrangement reinforced the anisotropy findings, including loss of transnuclear stress fibers, ring-like actin formation, and ARC formation [214,216-219]. Multivariant logistical regression of phenotype frequency was used to determine statistical certainty of the dichotomous outcome and clearly showed that podocytes expressing ABIN1[D472N] were more likely to produced aberrant actin structures under control conditions and that development of these structures was even more frequent following 24 hours of exposure to a degranulated neutrophil supernatant. In total, actin rearrangement was strongly associated to ABIN1 genotype in cultured podocytes in a way that induced pathologic changes, both under stress and at baseline, which would promote a greater propensity for worsening disease both before and after a nephrotoxic insult.

When considering that podocytes can both recruit and activate neutrophils and that they undergo pathogenic responses as a result of exposure to granules released by activated neutrophils, there is clearly support for a mechanism of GN involving a podocyte-neutrophil axis of pathological interactions which promote proteinurea and worsening renal function. Furthermore, functional ABIN1 seems to be important in the regulation of podocyte responses to inhibit the damaging activity of this proinflammatory axis. These data support a novel pathogenic mechanism for GN involving accelerated 
neutrophil recruitment and activation through proinflammatory signaling within podocytes expressing aberrant ABIN1. These pathogenic processes described above represent a shift from the current paradigm of GN pathology shown in Figure 4. The findings of this dissertation are outlined graphically in the form of a healthy glomerulus (Figure 41) and an inflamed glomerulus (Figure 42) and aim to show the importance of podocytes in the pathogenesis of GN.

In closing, there is growing evidence for intraglomerular NF- $\mathrm{kB}$ activation and genetic variation in the upstream NF- $\mathrm{BB}$ inhibitor ABIN1 in the pathogenesis of GN [33,34,53,184,220,221]. Current treatment for GN involves systemic administration of large doses of corticosteroids, immunosuppressive drugs, and/or biologic agents. Those drugs are successful in inducing a remission of disease in only about $50 \%$ of patients, and those drugs are associated with significant side effects [222,223]. Our findings in this

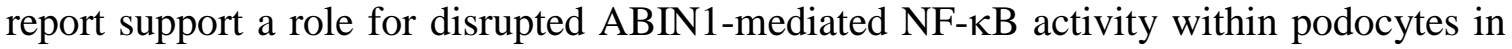
the proinflammatory activation and injury commonly described in GN. This indicates a plausible therapeutic impact for the development of a kidney-targeted NF- $\mathrm{BB}$ inhibitor, especially for treatment of GN patients with genetic variants for ABIN1 or other NF-kB regulators. We also show that a peptide inhibitor of neutrophil degranulation impeded the development of proteinuria in an acute model of immune complex-mediated GN, warranting further testing of this biologic in more severe, chronic GN models. 


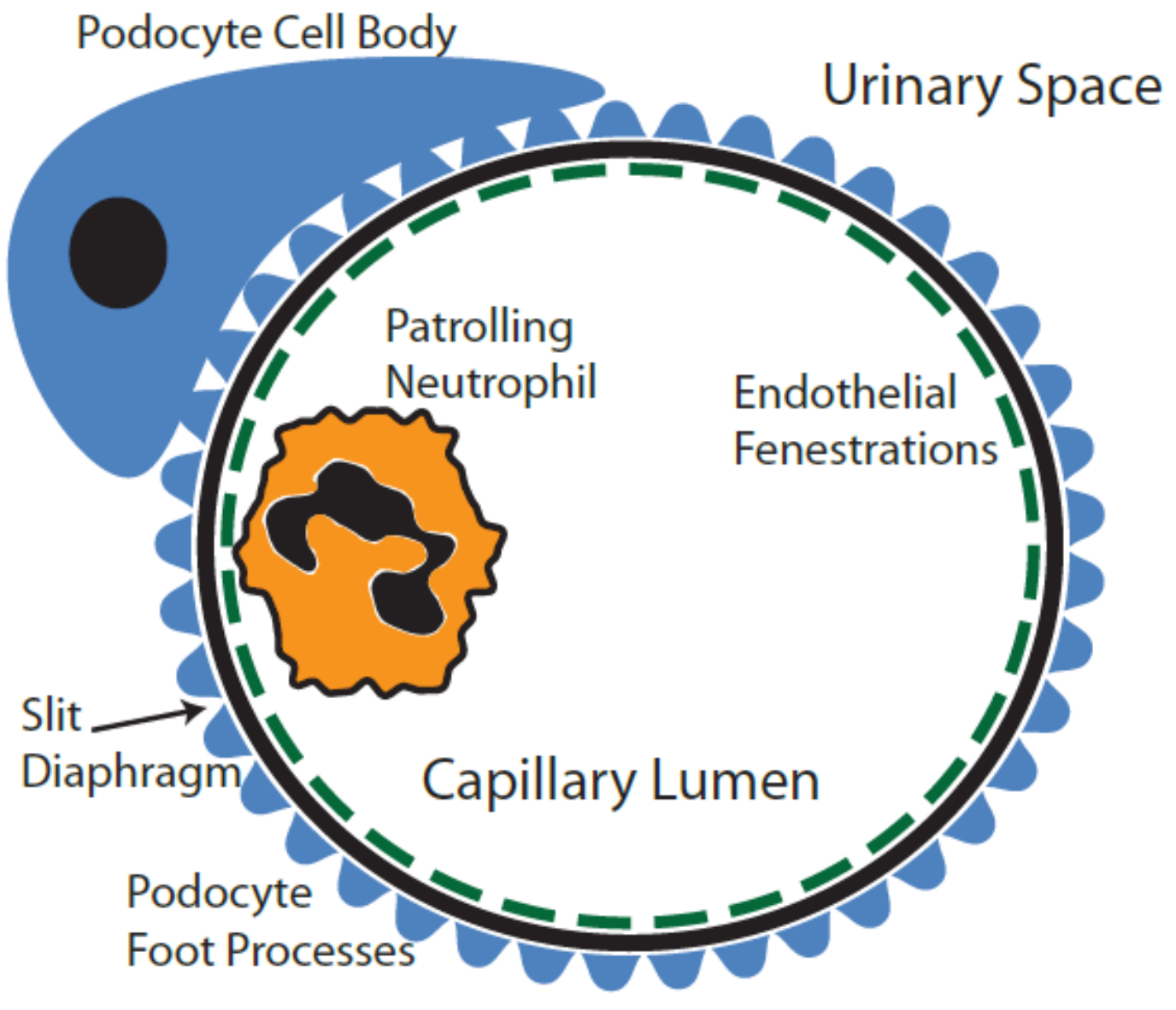

Figure 41 - Healthy Glomerular Capillary

This diagram represents the glomerular capillary in a healthy glomerulus. Podocyte foot processes are evenly spaced with well-defined slit diaphragms and the entire glomerular basement membrane is enveloped in foot processes. Neutrophils patrol the glomerulus constitutively but are not activated.

Korte, unpublished. 


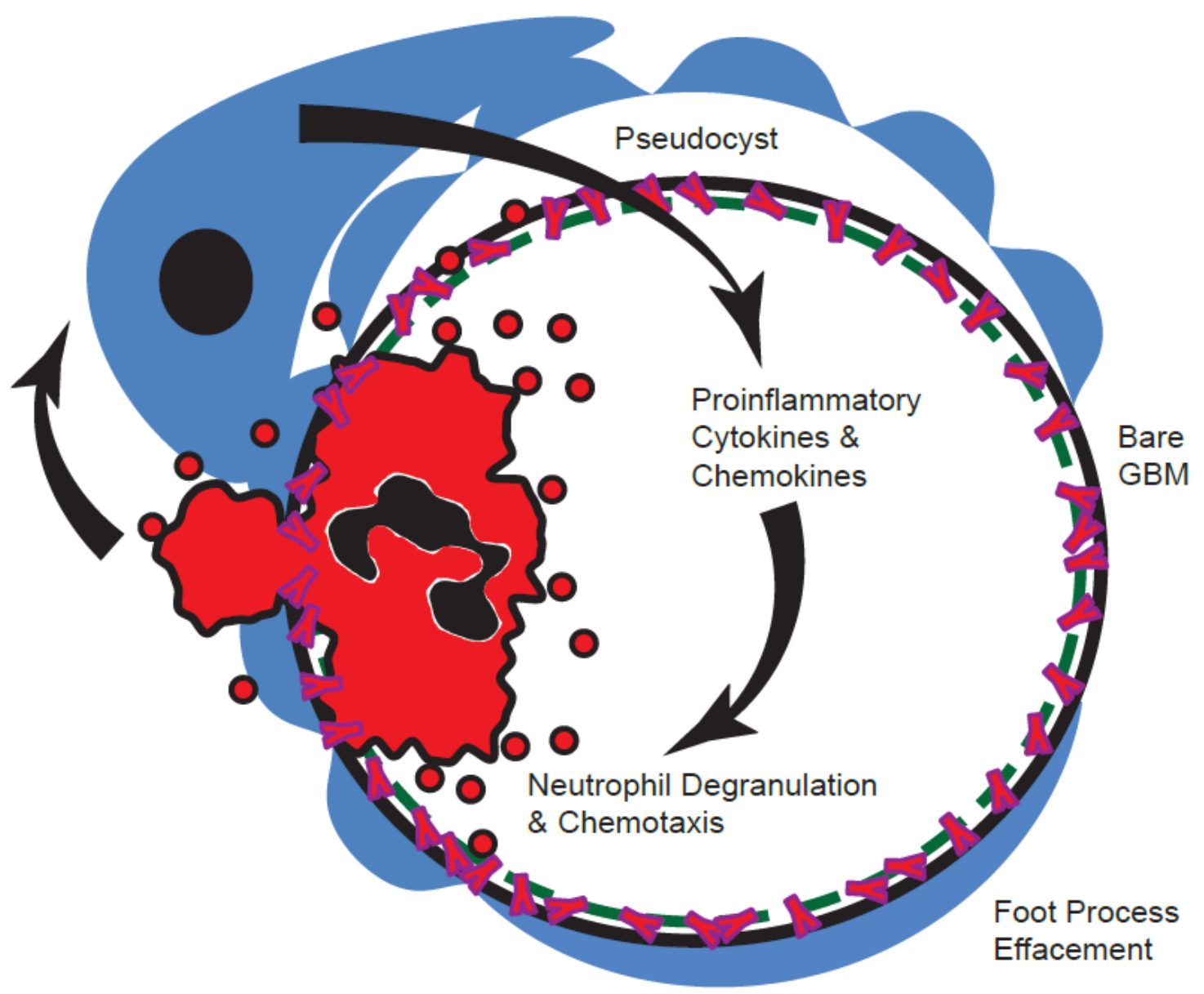

Figure 42 - Inflamed Glomerular Capillary

This diagram represents the proposed pro-inflammatory podocyte-neutrophil axis in the pathogenesis of glomerulonephritis. Antibody-mediated injury is augmented by NF-кBdirected inflammatory injury in the glomerulus which includes neutrophil chemotaxis, activation and degranulation, podocyte foot process effacement and shedding, leaving bare GBM, and pseudocyst formation. Our findings suggest that these injurious events are mediated by loss of podocyte ABIN1 ubiquitin binding activity ensued by genetic variants.

Korte, unpublished. 


\section{CHAPTER VI}

\section{CONCLUSION}

This work describes a novel and underappreciated cellular interaction leading to glomerulonephritis and describes the molecular mechanism by which it occurs. It reveals that podocytes are not the passive victims of glomerular injury as they have long been described. Podocytes are integral players within the glomerulus and have the ability to orchestrate changes in the molecular microenvironment which affect both themselves and the kidney as a whole. Mechanistically, this work shows an important role of ABIN1 as a critical inhibitor of NF- $\kappa \mathrm{B}$ in glomerular disease, and suggests polymorphisms in ABIN1 could predispose a patient to a number of diseases involving enhanced NF- $\mathrm{B}$ stimulation. And finally, it has shed light on early changes which occur following glomerular damage which initiate later pathology. Early recognition and inhibition of these early events may reduce or prevent the development of chronic glomerular disease in some patients.

It is my hope that this work will inspire future study in the function of ABIN1, glomerular disease and the potential for target tissues to provide inflammatory control mechanisms for what have long been thought to be immune-mediated diseases. To facilitate the development of subsequent projects, I have described in detail the beneficial function of mass spectrometry based techniques had have on discovery and outlined 
specifically how it was used in this project to identify physiologically important proteins from complex samples. Although in vivo models invariably present the most physiologically accurate data pertaining to any physiological process, it can be overwhelmingly difficult or impossible to obtain pure samples from single-cell populations. The combination of in vitro experimentation with relatively unbiased analysis methods can elucidate the importance of low abundant factors in disease that would certainly be lost among the noise of a more complex system.

Looking beyond this work, there are specific areas of study I hope to promote through the publication of this dissertation. The history of ABIN1 following its initial discovery and progressing into transgenic mouse models highlighted the complexity and promiscuity of ABIN1 as a ubiquitin binding protein. A list of ABIN1 interacting partners is likely vast, and will need to be identified within individual cell types before we may begin to understand the full influence ABIN1 has on human disease and homeostasis. Even when looking specifically at one cytokine (TNF- $\alpha$ ) as it stimulates one specific pathway (NF- $\mathrm{B})$, there are three different polyubiquitin linkages interacting involving multiple proteins, all of which have different roles and all of which become possible targets of ABIN1 binding. When considering the cell as a whole which may contain thousands of proteins regulated by ubiquitin binding, the potential of ABIN1 activity in any cell type becomes immense. Future studies can begin by using coimmunoprecipitation of ABIN1 and its interacting partners followed by identification using LC-MS/MS. Tissue specific polymorphisms exhibiting increased risk of disease, as described in the GWAS study in Chapter 4, highlight promising initial directions for this type of discovery. Importantly, these novel interactions may involve tissue specific 
pathways which harbor potent targets for precise and high affinity small-molecule therapeutics to treat disease only in the target tissue type, which could reduce off-target effects of systemic therapies in use today. 


\section{REFERENCES}

1 Ortega, L. M., Schultz, D. R., Lenz, O., Pardo, V. and Contreras, G. N. (2010) Review: Lupus nephritis: pathologic features, epidemiology and a guide to therapeutic decisions. Lupus 19, 557.

2 Floege, J. and Amann, K. (2016) Primary glomerulonephritides. The Lancet.

3 Jennette, J. C. and Falk, R. J. (1998) Pathogenesis of the vascular and glomerular damage in ANCA-positive vasculitis. Nephrol. Dial. Transplant. Off. Publ. Eur. Dial. Transpl. Assoc. - Eur. Ren. Assoc. 13 Suppl 1, 16-20.

4 Borchers, A. T., Leibushor, N., Naguwa, S. M., Cheema, G. S., Shoenfeld, Y. and Gershwin, M. E. (2012) Lupus nephritis: A critical review. Autoimmun Rev 12, 174-94.

5 Beck Jr, L. H., Bonegio, R. G. ., Lambeau, G., Beck, D. M., Powell, D. W., Cummins, T. D., Klein, J. B. and Salant, D. J. (2009) M-type phospholipase A2 receptor as target antigen in idiopathic membranous nephropathy. N. Engl. J. Med. $361,11-21$.

6 Goodpasture, E. W. (1919) THE SIGNIFICANCE OF CERTAIN PULMONARY LESIONS IN RELATION TO THE ETIOLOGY OF INFLUENZA: Am. J. Med. Sci. 158, 863-870.

7 Bagavant, H. and Fu, S. M. (2009) Pathogenesis of kidney disease in systemic lupus erythematosus. Curr. Opin. Rheumatol. 21, 489-494.

8 Tsokos, G. C. (2011) Systemic lupus erythematosus. N Engl J Med 365, 2110-21. 
9 Lahita, R. G. (Ed.). (2011) Systemic lupus erythematosus 5th ed., Elsevier Academic Press, Amsterdam; New York.

10 Weening, J. J., D D’AGATI, V., Schwartz, M. M., Seshan, S. V., Alpers, C. E., Appel, G. B., Balow, J. E., Bruijn, J. A., Cook, T., Ferrario, F., et al. (2004) The classification of glomerulonephritis in systemic lupus erythematosus revisited. Kidney Int. 65, 521-530.

11 Cervera, R., Khamashta, M. A., Font, J., Sebastiani, G. D., Gil, A., Lavilla, P., Aydintug, A. O., Jedryka-Góral, A., de Ramón, E., Fernández-Nebro, A., et al. (1999) Morbidity and mortality in systemic lupus erythematosus during a 5-year period. A multicenter prospective study of 1,000 patients. European Working Party on Systemic Lupus Erythematosus. Medicine (Baltimore) 78, 167-175.

12 Molino, C., Fabbian, F. and Longhini, C. (2009) Clinical approach to lupus nephritis: recent advances. Eur. J. Intern. Med. 20, 447-453.

13 Rahman, A. and Isenberg, D. A. (2008) Systemic lupus erythematosus. N. Engl. J. Med. 358, 929-939.

14 Buhaescu, I., Covic, A. and Deray, G. (2007) Treatment of proliferative lupus nephritis--a critical approach. Semin Arthritis Rheum 36, 224-37.

15 Iwata, Y., Furuichi, K., Kaneko, S. and Wada, T. (2011) The Role of Cytokine in the Lupus Nephritis. J. Biomed. Biotechnol. 2011, 1-7.

16 Robbins, S. L., Kumar, V. and Cotran, R. S. (Eds.). (2010) Robbins and Cotran pathologic basis of disease 8th ed., Saunders/Elsevier, Philadelphia, PA. 
17 Kriz, W., Shirato, I., Nagata, M., LeHir, M. and Lemley, K. V. (2013) The podocyte's response to stress: the enigma of foot process effacement. Am. J. Physiol. Renal Physiol. 304, F333-347.

18 Allam, R. and Anders, H.-J. (2008) The role of innate immunity in autoimmune tissue injury. Curr. Opin. Rheumatol. 20, 538-544.

19 Wu, S.-H., Lu, C., Dong, L. and Chen, Z.-Q. (2008) Signal transduction involved in CTGF-induced production of chemokines in mesangial cells. Growth Factors Chur Switz. 26, 192-200.

20 Yu, D., Petermann, A., Kunter, U., Rong, S., Shankland, S. J. and Floege, J. (2005) Urinary Podocyte Loss Is a More Specific Marker of Ongoing Glomerular Damage than Proteinuria. J. Am. Soc. Nephrol. 16, 1733-1741.

21 Sato, Y., Wharram, B. L., Lee, S. K., Wickman, L., Goyal, M., Venkatareddy, M., Chang, J. W., Wiggins, J. E., Lienczewski, C., Kretzler, M., et al. (2009) Urine Podocyte mRNAs Mark Progression of Renal Disease. J. Am. Soc. Nephrol. JASN 20, 1041-1052.

22 Hewins, P., Morgan, M. D., Holden, N., Neil, D., Williams, J. M., Savage, C. O. S. and Harper, L. (2006) IL-18 is upregulated in the kidney and primes neutrophil responsiveness in ANCA-associated vasculitis. Kidney Int. 69, 605-615.

23 Kuravi, S. J., McGettrick, H. M., Satchell, S. C., Saleem, M. A., Harper, L., Williams, J. M., Rainger, G. E. and Savage, C. O. S. (2014) Podocytes Regulate Neutrophil Recruitment by Glomerular Endothelial Cells via IL-6-Mediated Crosstalk. J. Immunol. 193, 234-243. 
24 Kniker, W. T. and Cochrane, C. G. (1965) PATHOGENIC FACTORS IN VASULAR LESIONS OF EXPERIMENTAL SERUM SICKNESS. J. Exp. Med. 122, 83-98.

25 Cochrane, C. G., Unanue, E. R. and Dixon, F. J. (1965) A ROLE OF POLYMORPHONUCLEAR LEUKOCYTES AND COMPLEMENT IN NEPHROTOXIC NEPHRITIS. J. Exp. Med. 122, 99-116.

26 Schrijver, G., Schalkwijk, J., Robben, J. C., Assmann, K. J. and Koene, R. A. (1989) Antiglomerular basement membrane nephritis in beige mice. Deficiency of leukocytic neutral proteinases prevents the induction of albuminuria in the heterologous phase. J. Exp. Med. 169, 1435-1448.

27 Schrijver, G., Bogman, M. J., Assmann, K. J., de Waal, R. M., Robben, H. C., van Gasteren, H. and Koene, R. A. (1990) Anti-GBM nephritis in the mouse: role of granulocytes in the heterologous phase. Kidney Int. 38, 86-95.

28 Devi, S., Li, A., Westhorpe, C. L. V., Lo, C. Y., Abeynaike, L. D., Snelgrove, S. L., Hall, P., Ooi, J. D., Sobey, C. G., Kitching, A. R., et al. (2013) Multiphoton imaging reveals a new leukocyte recruitment paradigm in the glomerulus. Nat. Med. 19, 107-112.

29 Kim, N. D. and Luster, A. D. (2015) The role of tissue resident cells in neutrophil recruitment. Trends Immunol. 36, 547-555.

30 Kalergis, A. M., Iruretagoyena, M. I., Barrientos, M. J., Gonzalez, P. A., Herrada, A. A., Leiva, E. D., Gutierrez, M. A., Riedel, C. A., Bueno, S. M. and Jacobelli, S. H. (2009) Modulation of nuclear factor-kappaB activity can influence the susceptibility to systemic lupus erythematosus. Immunology 128, e306-14. 
31 Kang, H. K., Ecklund, D., Liu, M. and Datta, S. K. (2009) Apigenin, a nonmutagenic dietary flavonoid, suppresses lupus by inhibiting autoantigen presentation for expansion of autoreactive Th1 and Th17 cells. Arthritis Res Ther 11, R59.

32 Zheng, L., Sinniah, R. and Hsu, S. I. (2006) Renal cell apoptosis and proliferation may be linked to nuclear factor-kappaB activation and expression of inducible nitric oxide synthase in patients with lupus nephritis. Hum Pathol 37, 637-47.

33 Zheng, L., Sinniah, R. and Hsu, S. I.-H. (2006) In situ glomerular expression of activated NF-kappaB in human lupus nephritis and other non-proliferative proteinuric glomerulopathy. Virchows Arch. Int. J. Pathol. 448, 172-183.

34 Zheng, L., Sinniah, R. and Hsu, S. I. (2008) Pathogenic role of NF-kappaB activation in tubulointerstitial inflammatory lesions in human lupus nephritis. $\mathrm{J}$ Histochem Cytochem 56, 517-29.

35 Bruggeman, L. A., Drawz, P. E., Kahoud, N., Lin, K., Barisoni, L. and Nelson, P. J. (2011) TNFR2 interposes the proliferative and NF- $\kappa$ B-mediated inflammatory response by podocytes to TNF- $\alpha$. Lab. Investig. J. Tech. Methods Pathol. 91, 413425.

36 Bao, H., Ge, Y., Peng, A. and Gong, R. (2015) Fine-tuning of NFkB by glycogen synthase kinase $3 \beta$ directs the fate of glomerular podocytes upon injury. Kidney Int. 87, 1176-1190.

37 Brähler, S., Ising, C., Hagmann, H., Rasmus, M., Hoehne, M., Kurschat, C., Kisner, T., Goebel, H., Shankland, S. J., Addicks, K., et al. (2012) Intrinsic 
proinflammatory signaling in podocytes contributes to podocyte damage and prolonged proteinuria. Am. J. Physiol. Renal Physiol.

38 Sakurai, H., Hisada, Y., Ueno, M., Sugiura, M., Kawashima, K. and Sugita, T. (1996) Activation of transcription factor NF-kappa B in experimental glomerulonephritis in rats. Biochim. Biophys. Acta 1316, 132-138.

39 Oeckinghaus, A., Hayden, M. S. and Ghosh, S. (2011) Crosstalk in NF-kappaB signaling pathways. Nat Immunol 12, 695-708.

40 Verma, I. M., Stevenson, J. K., Schwarz, E. M., Van Antwerp, D. and Miyamoto, S. (1995) Rel/NF-kappa B/I kappa B family: intimate tales of association and dissociation. Genes Dev 9, 2723-35.

41 Jacobs, M. D. and Harrison, S. C. (1998) Structure of an IkappaBalpha/NF-kappaB complex. Cell 95, 749-58.

42 Karin, M. (1999) How NF-kappaB is activated: the role of the IkappaB kinase (IKK) complex. Oncogene 18, 6867-74.

43 Walczak, H., Iwai, K. and Dikic, I. (2012) Generation and physiological roles of linear ubiquitin chains. BMC Biol 10, 23.

44 Tokunaga, F., Sakata, S., Saeki, Y., Satomi, Y., Kirisako, T., Kamei, K., Nakagawa, T., Kato, M., Murata, S., Yamaoka, S., et al. (2009) Involvement of linear polyubiquitylation of NEMO in NF-kappaB activation. Nat. Cell Biol. 11, 123-132.

45 Adhikari, A., Xu, M. and Chen, Z. J. (2007) Ubiquitin-mediated activation of TAK1 and IKK. Oncogene 26, 3214-26. 
46 Yamamoto, M., Sato, S., Hemmi, H., Hoshino, K., Kaisho, T., Sanjo, H., Takeuchi, O., Sugiyama, M., Okabe, M., Takeda, K., et al. (2003) Role of Adaptor TRIF in the MyD88-Independent Toll-Like Receptor Signaling Pathway. Science 301, 640-643.

47 Rahighi, S., Ikeda, F., Kawasaki, M., Akutsu, M., Suzuki, N., Kato, R., Kensche, T., Uejima, T., Bloor, S., Komander, D., et al. (2009) Specific recognition of linear ubiquitin chains by NEMO is important for NF-kappaB activation. Cell 136, 1098109.

48 Haas, T. L., Emmerich, C. H., Gerlach, B., Schmukle, A. C., Cordier, S. M., Rieser, E., Feltham, R., Vince, J., Warnken, U., Wenger, T., et al. (2009) Recruitment of the linear ubiquitin chain assembly complex stabilizes the TNF-R1 signaling complex and is required for TNF-mediated gene induction. Mol Cell 36, 831-44.

49 Heyninck, K., De Valck, D., Vanden Berghe, W., Van Criekinge, W., Contreras, R., Fiers, W., Haegeman, G. and Beyaert, R. (1999) The zinc finger protein A20 inhibits TNF-induced NF-kappaB-dependent gene expression by interfering with an RIP- or TRAF2-mediated transactivation signal and directly binds to a novel NFkappaB-inhibiting protein ABIN. J. Cell Biol. 145, 1471-1482.

50 Heyninck, K., Kreike, M. M. and Beyaert, R. (2003) Structure-function analysis of the A20-binding inhibitor of NF-kappa B activation, ABIN-1. FEBS Lett. 536, 135140.

51 Oshima, S., Turer, E. E., Callahan, J. A., Chai, S., Advincula, R., Barrera, J., Shifrin, N., Lee, B., Yen, B., Woo, T., et al. (2008) ABIN-1 is a ubiquitin sensor that restricts cell death and sustains embryonic development. Nature 457, 906-909. 
52 Zhou, J., Wu, R., High, A. A., Slaughter, C. A., Finkelstein, D., Rehg, J. E., Redecke, V. and Häcker, H. (2011) A20-binding inhibitor of NF-кB (ABIN1) controls Toll-like receptor-mediated CCAAT/enhancer-binding protein $\beta$ activation and protects from inflammatory disease. Proc. Natl. Acad. Sci. U. S. A. 108, E9981006.

53 Nanda, S. K., Venigalla, R. K. C., Ordureau, A., Patterson-Kane, J. C., Powell, D. W., Toth, R., C. Arthur, J. S. and Cohen, P. (2011) Polyubiquitin binding to ABIN1 is required to prevent autoimmunity. J. Exp. Med. 208, 1215 -1228.

54 Vereecke, L., Beyaert, R. and van Loo, G. (2009) The ubiquitin-editing enzyme A20 (TNFAIP3) is a central regulator of immunopathology. Trends Immunol. 30, 383-391.

55 Verstrepen, L., Carpentier, I., Verhelst, K. and Beyaert, R. (2009) ABINs: A20 binding inhibitors of NF-kappa B and apoptosis signaling. Biochem Pharmacol 78, 105-14.

56 Conze, D. B., Wu, C. J., Thomas, J. A., Landstrom, A. and Ashwell, J. D. (2008) Lys63-linked polyubiquitination of IRAK-1 is required for interleukin-1 receptorand toll-like receptor-mediated NF-kappaB activation. Mol Cell Biol 28, 3538-47.

57 Laplantine, E., Fontan, E., Chiaravalli, J., Lopez, T., Lakisic, G., Veron, M., Agou, F. and Israel, A. (2009) NEMO specifically recognizes K63-linked poly-ubiquitin chains through a new bipartite ubiquitin-binding domain. EMBO J 28, 2885-95.

58 Windheim, M., Stafford, M., Peggie, M. and Cohen, P. (2008) Interleukin-1 (IL-1) induces the Lys63-linked polyubiquitination of IL-1 receptor-associated kinase 1 to 
facilitate NEMO binding and the activation of IkappaBalpha kinase. Mol Cell Biol 28, 1783-91.

59 Mauro, C., Pacifico, F., Lavorgna, A., Mellone, S., Iannetti, A., Acquaviva, R., Formisano, S., Vito, P. and Leonardi, A. (2006) ABIN-1 binds to NEMO/IKKgamma and co-operates with A20 in inhibiting NF-kappaB. J. Biol. Chem. 281, 18482-18488.

60 Evans, P. C., Ovaa, H., Hamon, M., Kilshaw, P. J., Hamm, S., Bauer, S., Ploegh, H. L. and Smith, T. S. (2004) Zinc-finger protein A20, a regulator of inflammation and cell survival, has de-ubiquitinating activity. Biochem J 378, 727-34.

61 Shembade, N., Ma, A. and Harhaj, E. W. (2010) Inhibition of NF-kappaB signaling by A20 through disruption of ubiquitin enzyme complexes. Science 327, 1135-9.

62 Wagner, S., Carpentier, I., Rogov, V., Kreike, M., Ikeda, F., Löhr, F., Wu, C.-J., Ashwell, J. D., Dötsch, V., Dikic, I., et al. (2008) Ubiquitin binding mediates the NF-kappaB inhibitory potential of ABIN proteins. Oncogene 27, 3739-3745.

63 Wiggins, J. E., Patel, S. R., Shedden, K. A., Goyal, M., Wharram, B. L., Martini, S., Kretzler, M. and Wiggins, R. C. (2010) NFkappaB promotes inflammation, coagulation, and fibrosis in the aging glomerulus. J. Am. Soc. Nephrol. JASN 21, $587-597$.

64 Hussain, S., Romio, L., Saleem, M., Mathieson, P., Serrano, M., Moscat, J., DiazMeco, M., Scambler, P. and Koziell, A. (2009) Nephrin deficiency activates NFkappaB and promotes glomerular injury. J. Am. Soc. Nephrol. JASN 20, 17331743. 
65 Adrianto, I., Wang, S., Wiley, G. B., Lessard, C. J., Kelly, J. A., Adler, A. J., Glenn, S. B., Williams, A. H., Ziegler, J. T., Comeau, M. E., et al. (2012) Two independent functional risk haplotypes in TNIP1 are associated with systemic lupus erythematosus. Arthritis Rheum.

66 Adrianto, I., Wen, F., Templeton, A., Wiley, G., King, J. B., Lessard, C. J., Bates, J. S., Hu, Y., Kelly, J. A., Kaufman, K. M., et al. (2011) Association Between a Functional Variant Downstream of TNFAIP3 and Systemic Lupus Erythematosus. Nat Genet 43, 253-258.

67 Gateva, V., Sandling, J. K., Hom, G., Taylor, K. E., Chung, S. A., Sun, X., Ortmann, W., Kosoy, R., Ferreira, R. C., Nordmark, G., et al. (2009) A large-scale replication study identifies TNIP1, PRDM1, JAZF1, UHRF1BP1 and IL10 as risk loci for systemic lupus erythematosus. Nat. Genet. 41, 1228-1233.

68 Han, J.-W., Zheng, H.-F., Cui, Y., Sun, L.-D., Ye, D.-Q., Hu, Z., Xu, J.-H., Cai, Z.M., Huang, W., Zhao, G.-P., et al. (2009) Genome-wide association study in a Chinese Han population identifies nine new susceptibility loci for systemic lupus erythematosus. Nat. Genet. 41, 1234-1237.

69 Zhong, H., Li, X. L., Li, M., Hao, L. X., Chen, R. W., Xiang, K., Qi, X. B., Ma, R. Z. and Su, B. (2011) Replicated associations of TNFAIP3, TNIP1 and ETS1 with systemic lupus erythematosus in a southwestern Chinese population. Arthritis Res Ther 13, R186.

70 Hochberg, M. C. (1997) Updating the American College of Rheumatology revised criteria for the classification of systemic lupus erythematosus. Arthritis Rheum 40, 1725. 
71 Allanore, Y., Saad, M., Dieude, P., Avouac, J., Distler, J. H., Amouyel, P., MatucciCerinic, M., Riemekasten, G., Airo, P., Melchers, I., et al. (2011) Genome-wide scan identifies TNIP1, PSORS1C1, and RHOB as novel risk loci for systemic sclerosis. PLoS Genet 7, e1002091.

72 Nair, R. P., Duffin, K. C., Helms, C., Ding, J., Stuart, P. E., Goldgar, D., Gudjonsson, J. E., Li, Y., Tejasvi, T., Feng, B. J., et al. (2009) Genome-wide scan reveals association of psoriasis with IL-23 and NF-kappaB pathways. Nat Genet 41, 199-204.

73 Sun, L. D., Cheng, H., Wang, Z. X., Zhang, A. P., Wang, P. G., Xu, J. H., Zhu, Q. X., Zhou, H. S., Ellinghaus, E., Zhang, F. R., et al. (2010) Association analyses identify six new psoriasis susceptibility loci in the Chinese population. Nat. Genet. 42, 1005-9.

74 Bowes, J., Orozco, G., Flynn, E., Ho, P., Brier, R., Marzo-Ortega, H., Coates, L., McManus, R., Ryan, A. W., Kane, D., et al. (2011) Confirmation of TNIP1 and IL23A as susceptibility loci for psoriatic arthritis. Ann Rheum Dis 70, 1641-4.

75 Hoggart, C. J., Parra, E. J., Shriver, M. D., Bonilla, C., Kittles, R. A., Clayton, D. G. and McKeigue, P. M. (2003) Control of confounding of genetic associations in stratified populations. Am. J. Hum. Genet. 72, 1492-1504.

76 Hoggart, C. J., Shriver, M. D., Kittles, R. A., Clayton, D. G. and McKeigue, P. M. (2004) Design and analysis of admixture mapping studies. Am. J. Hum. Genet. 74, 965-978. 
77 Price, A. L., Patterson, N. J., Plenge, R. M., Weinblatt, M. E., Shadick, N. A. and Reich, D. (2006) Principal components analysis corrects for stratification in genome-wide association studies. Nat. Genet. 38, 904-909.

78 Lessard, C. J., Adrianto, I., Kelly, J. A., Kaufman, K. M., Grundahl, K. M., Adler, A., Williams, A. H., Gallant, C. J., Anaya, J. M., Bae, S. C., et al. (2011) Identification of a systemic lupus erythematosus susceptibility locus at 11p13 between PDHX and CD44 in a multiethnic study. Am J Hum Genet 88, 83-91.

79 Purcell, S., Neale, B., Todd-Brown, K., Thomas, L., Ferreira, M. A., Bender, D., Maller, J., Sklar, P., de Bakker, P. I., Daly, M. J., et al. (2007) PLINK: a tool set for whole-genome association and population-based linkage analyses. Am J Hum Genet 81, 559-75.

80 Smith, M. W., Patterson, N., Lautenberger, J. A., Truelove, A. L., McDonald, G. J., Waliszewska, A., Kessing, B. D., Malasky, M. J., Scafe, C., Le, E., et al. (2004) A high-density admixture map for disease gene discovery in african americans. Am J Hum Genet 74, 1001-13.

81 Halder, I., Shriver, M., Thomas, M., Fernandez, J. R. and Frudakis, T. (2008) A panel of ancestry informative markers for estimating individual biogeographical ancestry and admixture from four continents: utility and applications. Hum Mutat 29, 648-58.

82 Saleem, M. A., O’Hare, M. J., Reiser, J., Coward, R. J., Inward, C. D., Farren, T., Xing, C. Y., Ni, L., Mathieson, P. W. and Mundel, P. (2002) A conditionally immortalized human podocyte cell line demonstrating nephrin and podocin expression. J. Am. Soc. Nephrol. JASN 13, 630-638. 
83 Barati, M. T., Gould, J. C., Salyer, S. A., Isaacs, S., Wilkey, D. W. and Merchant, M. L. (2016) Influence of Acute High Glucose on Protein Abundance Changes in Murine Glomerular Mesangial Cells. J. Diabetes Res. 2016.

84 Boudaoud, A., Burian, A., Borowska-Wykręt, D., Uyttewaal, M., Wrzalik, R., Kwiatkowska, D. and Hamant, O. (2014) FibrilTool, an ImageJ plug-in to quantify fibrillar structures in raw microscopy images. Nat. Protoc. 9, 457-463.

85 Nesvizhskii, A. I., Keller, A., Kolker, E. and Aebersold, R. (2003) A statistical model for identifying proteins by tandem mass spectrometry. Anal. Chem. 75, 4646-4658.

86 Cummins, T. D., Barati, M. T., Coventry, S. C., Salyer, S. A., Klein, J. B. and Powell, D. W. (2010) Quantitative mass spectrometry of diabetic kidney tubules identifies GRAP as a novel regulator of TGF-[beta] signaling. Biochim. Biophys. Acta BBA-Proteins Proteomics 1804, 653-661.

87 Powell, D. W., Weaver, C. M., Jennings, J. L., McAfee, K. J., He, Y., Weil, P. A. and Link, A. J. (2004) Cluster analysis of mass spectrometry data reveals a novel component of SAGA. Mol. Cell. Biol. 24, 7249.

88 Ma, X., Conklin, D. J., Li, F., Dai, Z., Hua, X., Li, Y., Xu-Monette, Z. Y., Young, K. H., Xiong, W., Wysoczynski, M., et al. (2015) The oncogenic microRNA miR21 promotes regulated necrosis in mice. Nat. Commun. 6, 7151.

89 McLeish, K. R., Uriarte, S. M., Tandon, S., Creed, T. M., Le, J. and Ward, R. A. (2013) Exocytosis of neutrophil granule subsets and activation of prolyl isomerase 1 are required for respiratory burst priming. J. Innate Immun. 5, 277-289. 
90 Link, A. J., Eng, J., Schieltz, D. M., Carmack, E., Mize, G. J., Morris, D. R., Garvik, B. M. and Yates, J. R., 3rd. (1999) Direct analysis of protein complexes using mass spectrometry. Nat. Biotechnol. 17, 676-682.

91 Powell, D. W., Merchant, M. L. and Link, A. J. (2006) Discovery of regulatory molecular events and biomarkers using 2D capillary chromatography and mass spectrometry. Expert Rev. Proteomics 3, 63-74.

92 Powell, D. W., Rane, M. J., Joughin, B. A., Kalmukova, R., Hong, J.-H., Tidor, B., Dean, W. L., Pierce, W. M., Klein, J. B., Yaffe, M. B., et al. (2003) Proteomic identification of 14-3-3zeta as a mitogen-activated protein kinase-activated protein kinase 2 substrate: role in dimer formation and ligand binding. Mol. Cell. Biol. 23, 5376-5387.

93 Clarke, C. H., Buckley, J. A. and Fung, E. T. (2005) SELDI-TOF-MS proteomics of breast cancer. Clin. Chem. Lab. Med. CCLM FESCC 43, 1314-1320.

94 Lu, X., Chen, X., Huang, L., Zhu, C., Gu, Y. and Ye, S. (2010) Anti-alphainternexin autoantibody from neuropsychiatric lupus induce cognitive damage via inhibiting axonal elongation and promote neuron apoptosis. PloS One 5, e11124.

95 Katsumata, Y., Kawaguchi, Y., Baba, S., Hattori, S., Tahara, K., Ito, K., Iwasaki, T., Yamaguchi, N., Oyama, M., Kozuka-Hata, H., et al. (2011) Identification of three new autoantibodies associated with systemic lupus erythematosus using two proteomic approaches. Mol. Cell. Proteomics MCP 10, M110.005330.

96 Seko, Y., Matsumoto, A., Fukuda, T., Imai, Y., Fujimura, T., Taka, H., Mineki, R., Murayama, K., Hirata, Y. and Nagai, R. (2007) A case of neonatal lupus 
erythematosus presenting delayed dilated cardiomyopathy with circulating autoantibody to annexin A6. Int. Heart. J. 48, 407-415.

97 Iaccarino, L., Bettio, S., Zen, M., Nalotto, L., Gatto, M., Ramonda, R., Punzi, L. and Doria, A. (2013) Premature coronary heart disease in SLE: can we prevent progression? Lupus 22, 1232-1242.

98 Manzi, S., Meilahn, E. N., Rairie, J. E., Conte, C. G., Medsger, T. A., Jr, JansenMcWilliams, L., D’Agostino, R. B. and Kuller, L. H. (1997) Age-specific incidence rates of myocardial infarction and angina in women with systemic lupus erythematosus: comparison with the Framingham Study. Am. J. Epidemiol. 145, 408-415.

99 Esdaile, J. M., Abrahamowicz, M., Grodzicky, T., Li, Y., Panaritis, C., du Berger, R., Côte, R., Grover, S. A., Fortin, P. R., Clarke, A. E., et al. (2001) Traditional Framingham risk factors fail to fully account for accelerated atherosclerosis in systemic lupus erythematosus. Arthritis Rheum. 44, 2331-2337.

100 Asanuma, Y., Oeser, A., Shintani, A. K., Turner, E., Olsen, N., Fazio, S., Linton, M. F., Raggi, P. and Stein, C. M. (2003) Premature Coronary-Artery Atherosclerosis in Systemic Lupus Erythematosus. N. Engl. J. Med. 349, 2407-2415.

101 Lawrence, R. C., Felson, D. T., Helmick, C. G., Arnold, L. M., Choi, H., Deyo, R. A., Gabriel, S., Hirsch, R., Hochberg, M. C., Hunder, G. G., et al. (2008) Estimates of the prevalence of arthritis and other rheumatic conditions in the United States: Part II. Arthritis Rheum. 58, 26-35. 
102 Faurschou, M., Mellemkjaer, L., Starklint, H., Kamper, A.-L., Tarp, U., Voss, A. and Jacobsen, S. (2011) High risk of ischemic heart disease in patients with lupus nephritis. J. Rheumatol. 38, 2400-2405.

103 Appenzeller, S., Pineau, C. and Clarke, A. (2011) Acute lupus myocarditis: Clinical features and outcome. Lupus 20, 981-988.

104 Wijetunga, M. and Rockson, S. (2002) Myocarditis in systemic lupus erythematosus. Am. J. Med. 113, 419-423.

105 Comín-Colet, J., Sánchez-Corral, M. A., Alegre-Sancho, J. J., Valverde, J., LópezGómez, D., Sabaté, X., Juan-Mas, A. and Esplugas, E. (2001) Complete heart block in an adult with systemic lupus erythematosus and recent onset of hydroxychloroquine therapy. Lupus 10, 59-62.

106 Bidani, A. K., Roberts, J. L., Schwartz, M. M. and Lewis, E. J. (1980) Immunopathology of cardiac lesions in fatal systemic lupus erythematosus. Am. J. Med. 69, 849-858.

107 Gazarian, M., Feldman, B. M., Benson, L. N., Gilday, D. L., Laxer, R. M. and Silverman, E. D. (1998) Assessment of myocardial perfusion and function in childhood systemic lupus erythematosus. J. Pediatr. 132, 109-116.

108 Kurien, B. T., Newland, J., Paczkowski, C., Moore, K. L. and Scofield, R. H. (2000) Association of neutropenia in systemic lupus erythematosus (SLE) with anti-Ro and binding of an immunologically cross-reactive neutrophil membrane antigen. Clin. Exp. Immunol. 120, 209-217.

109 Wang, L., Dai, Y., Qi, S., Sun, B., Wen, J., Zhang, L. and Tu, Z. (2010) Comparative proteome analysis of peripheral blood mononuclear cells in systemic 
lupus erythematosus with iTRAQ quantitative proteomics. Rheumatol. Int. 2012 Mar;32(3):585-93.

110 Ottosson, L., Salomonsson, S., Hennig, J., Sonesson, S.-E., Dörner, T., Raats, J., Kuchroo, V. K., Sunnerhagen, M. and Wahren-Herlenius, M. (2005) Structurally derived mutations define congenital heart block-related epitopes within the 200-239 amino acid stretch of the Ro52 protein. Scand. J. Immunol. 61, 109-118.

111 Serada, S., Fujimoto, M., Takahashi, T., He, P., Hayashi, A., Tanaka, T., Hagihara, K., Yamadori, T., Mochizuki, M., Norioka, N., et al. (2007) Proteomic analysis of autoantigens associated with systemic lupus erythematosus: Anti-aldolase A antibody as a potential marker of lupus nephritis. PROTEOMICS-Clinical Appl. 1, $185-191$.

112 Pavon EJ, Munoz P, Lario A, Longobardo V, Carrascal M, Abi $\tilde{A}_{i} \mathrm{~J}$, Martin AB, Arias SA, Callejas-Rubio JL, Sola R, et al. (2006) Proteomic analysis of plasma from patients with systemic lupus erythematosus: increased presence of haptoglobin alpha2 polypeptide chains over the alpha1 isoforms. Proteomics 6 Suppl 1, S282S292.

113 Van Vlierberghe, H., Langlois, M. and Delanghe, J. (2004) Haptoglobin polymorphisms and iron homeostasis in health and in disease. Clin. Chim. Acta Int. J. Clin. Chem. 345, 35-42.

114 Chapelle, J. P., Albert, A., Smeets, J. P., Heusghem, C. and Kulbertus, H. E. (1982) Effect of the haptoglobin phenotype on the size of a myocardial infarct. N. Engl. J. Med. 307, 457-463. 
115 Reed, J. H., Neufing, P. J., Jackson, M. W., Clancy, R. M., Macardle, P. J., Buyon, J. P. and Gordon, T. P. (2007) Different temporal expression of immunodominant Ro60/60 kDa-SSA and La/SSB apotopes. Clin. Exp. Immunol. 148, 153-160.

116 Briassouli, P., Komissarova, E. V., Clancy, R. M. and Buyon, J. P. (2010) Role of the urokinase plasminogen activator receptor in mediating impaired efferocytosis of anti-SSA/Ro-bound apoptotic cardiocytes: Implications in the pathogenesis of congenital heart block. Circ. Res. 107, 374-387.

117 Gunteski-Hamblin, A. M., Song, G., Walsh, R. A., Frenzke, M., Boivin, G. P., Dorn, G. W., 2nd, Kaetzel, M. A., Horseman, N. D. and Dedman, J. R. (1996) Annexin VI overexpression targeted to heart alters cardiomyocyte function in transgenic mice. Am. J. Physiol. 270, H1091-1100.

118 Dai, Y., Hu, C., Huang, Y., Huang, H., Liu, J. and Lv, T. (2008) A proteomic study of peripheral blood mononuclear cells in systemic lupus erythematosus. Lupus 17, 799-804.

119 Li, T., Zheng, B., Huang, Z., Lin, Q., Zhao, L., Liao, Z., Zhao, J., Lin, Z. and Gu, J. (2010) Screening disease-associated proteins from sera of patients with rheumatoid arthritis: a comparative proteomic study. Chin. Med. J. (Engl.) 123, 537-543.

120 Cui, Y., Huang, M., He, Y., Zhang, S. and Luo, Y. (2011) Genetic ablation of apolipoprotein A-IV accelerates Alzheimer's disease pathogenesis in a mouse model. Am. J. Pathol. 178, 1298-1308.

121 Yi, L., Zeng, X., Tan, H., Ge, L., Ji, X.-X., Lin, M. and Su, Q. (2009) Proteomics analysis of apoptosis initiation induced by diallyl disulfide in human leukemia HL60 cells. Ai Zheng Aizheng Chin. J. Cancer 28, 33-37. 
122 Ohsawa, S., Watanabe, T., Katada, T., Nishina, H. and Miura, M. (2008) Novel antibody to human BASP1 labels apoptotic cells post-caspase activation. Biochem. Biophys. Res. Commun. 371, 639-643.

123 Kimura, A., Kanoh, Y., Sakurai, T., Koumura, A., Yamada, M., Hayashi, Y., Tanaka, Y., Hozumi, I., Takemura, M., Seishima, M., et al. (2010) Antibodies in patients with neuropsychiatric systemic lupus erythematosus. Neurology 74, 1372.

124 Kimura, A., Sakurai, T., Yamada, M., Koumura, A., Hayashi, Y., Tanaka, Y., Hozumi, I., Takemura, M., Seishima, M. and Inuzuka, T. (2010) Elevated Anti-Heat Shock Protein 60 Antibody Titer is Related to White Matter Hyperintensities. J. Stroke Cerebrovasc. Dis. Off. J. Natl. Stroke Assoc.

125 Reyes-Thomas, J., Blanco, I. and Putterman, C. (2010) Urinary Biomarkers in Lupus Nephritis. Clin. Rev. Allergy Immunol. 40, 138-150.

126 Oates, J. C., Varghese, S., Bland, A. M., Taylor, T. P., Self, S. E., Stanislaus, R., Almeida, J. S. and Arthur, J. M. (2005) Prediction of urinary protein markers in lupus nephritis. Kidney Int. 68, 2588-2592.

127 Mosley, K., Tam, F. W. K., Edwards, R. J., Crozier, J., Pusey, C. D. and Lightstone, L. (2006) Urinary proteomic profiles distinguish between active and inactive lupus nephritis. Rheumatology 45, $1497-1504$.

128 Zhang, X., Jin, M., Wu, H., Nadasdy, T., Nadasdy, G., Harris, N., Green-Church, K., Nagaraja, H., Birmingham, D. J., Yu, C.-Y., et al. (2008) Biomarker Discovery for Lupus Nephritis Through Longitudinal Urine Proteomics. Kidney Int. 74, 799_ 807. 
129 Zhang, X. and Rovin, B. H. (2010) Hepcidin expression by human monocytes in response to adhesion and pro-inflammatory cytokines. Biochim. Biophys. Acta 1800, $1262-1267$.

130 Suzuki, M., Ross, G. F., Wiers, K., Nelson, S., Bennett, M., Passo, M. H., Devarajan, P. and Brunner, H. I. (2007) Identification of a urinary proteomic signature for lupus nephritis in children. Pediatr. Nephrol. Berl. Ger. 22, 2047-2057.

131 Suzuki, M., Wiers, K., Brooks, E. B., Greis, K. D., Haines, K., Klein-Gitelman, M. S., Olson, J., Onel, K., O’Neil, K. M., Silverman, E. D., et al. (2009) Initial Validation of a Novel Protein Biomarker Panel for Active Pediatric Lupus Nephritis. Pediatr. Res. 65, 530-536.

132 Varghese, S. A., Powell, T. B., Budisavljevic, M. N., Oates, J. C., Raymond, J. R., Almeida, J. S. and Arthur, J. M. (2007) Urine Biomarkers Predict the Cause of Glomerular Disease. J. Am. Soc. Nephrol. JASN 18, 913-922.

133 Dong, J., Li, H., Wang, J.-B., Yao, Y. and Yang, Q.-R. (2011) Predictors for neuropsychiatric development in Chinese adolescents with systemic lupus erythematosus. Rheumatol. Int.

134 Sibbitt, W. L., Jr, Brandt, J. R., Johnson, C. R., Maldonado, M. E., Patel, S. R., Ford, C. C., Bankhurst, A. D. and Brooks, W. M. (2002) The incidence and prevalence of neuropsychiatric syndromes in pediatric onset systemic lupus erythematosus. J. Rheumatol. 29, 1536-1542.

135 Kimura, A., Sakurai, T., Tanaka, Y., Hozumi, I., Takahashi, K., Takemura, M., Saito, K., Seishima, M. and Inuzuka, T. (2008) Proteomic analysis of autoantibodies 
in neuropsychiatric systemic lupus erythematosus patient with white matter hyperintensities on brain MRI. Lupus 17, 16-20.

136 Ndhlovu, M., Preuss, B. E., Dengjel, J., Stevanovic, S., Weiner, S. M. and Klein, R. (2011) Identification of $\alpha$-tubulin as an autoantigen recognized by sera from patients with neuropsychiatric systemic lupus erythematosus. Brain. Behav. Immun. 25, 279-285.

137 Zhang, Y., Wang, J., Xiang, D., Wang, D. and Xin, X. (2009) Alterations in the expression of the apurinic/apyrimidinic endonuclease-1/redox factor-1 (APE1/Ref1) in human ovarian cancer and indentification of the therapeutic potential of APE1/Ref-1 inhibitor. Int. J. Oncol. 35, 1069-1079.

138 Johnson, R. B., Fearon, K., Mason, T. and Jindal, S. (1989) Cloning and characterization of the yeast chaperonin HSP60 gene. Gene 84, 295-302.

139 Zhu, J., Quyyumi, A. A., Rott, D., Csako, G., Wu, H., Halcox, J. and Epstein, S. E. (2001) Antibodies to human heat-shock protein 60 are associated with the presence and severity of coronary artery disease: evidence for an autoimmune component of atherogenesis. Circulation 103, 1071-1075.

140 Kao, S.-H., Hsu, T.-C., Yu, J.-S., Chen, J.-T., Li, S.-L., Lai, W.-X. and Tzang, B.-S. (2010) Proteomic analysis for the anti-apoptotic effects of cystamine on apoptosisprone macrophage. J. Cell. Biochem. 110, 660-670.

141 Dardik, R. and Inbal, A. (2006) Complex formation between tissue transglutaminase II (tTG) and vascular endothelial growth factor receptor 2 (VEGFR-2): proposed mechanism for modulation of endothelial cell response to VEGF. Exp. Cell Res. 312, 2973-2982. 
142 Pillai, A., Veeranan-Karmegam, R., Dhandapani, K. M. and Mahadik, S. P. (2008) Cystamine prevents haloperidol-induced decrease of BDNF/TrkB signaling in mouse frontal cortex. J. Neurochem. 107, 941-951.

143 D’souza, A., Kurien, B. T., Rodgers, R., Shenoi, J., Kurono, S., Matsumoto, H., Hensley, K., Nath, S. K. and Scofield, R. H. (2008) Detection of catalase as a major protein target of the lipid peroxidation product 4-HNE and the lack of its genetic association as a risk factor in SLE. BMC Med. Genet. 9, 62.

144 Ye, S., Pang, H., Gu, Y. Y., Hua, J., Chen, X. G., Bao, C. D., Wang, Y., Zhang, W., Qian, J., Tsao, B. P., et al. (2003) Protein interaction for an interferon-inducible systemic lupus associated gene, IFIT1. Rheumatology 42, 1155.

145 Walters, E., Rider, V., Abdou, N. I., Greenwell, C., Svojanovsky, S., Smith, P. and Kimler, B. F. (2009) Estradiol targets T cell signaling pathways in human systemic lupus. Clin. Immunol. Orlando Fla 133, 428-436.

146 Pachner, A. R., Warth, J. D., Pace, A. and Goelz, S. (2009) Effect of neutralizing antibodies on biomarker responses to interferon beta: the INSIGHT study. Neurology 73, 1493-1500.

147 Higgs, B. W., Liu, Z., White, B., Zhu, W., White, W. I., Morehouse, C., Brohawn, P., Kiener, P. A., Richman, L., Fiorentino, D., et al. (2011) Patients with systemic lupus erythematosus, myositis, rheumatoid arthritis and scleroderma share activation of a common type I interferon pathway. Ann. Rheum. Dis.

148 Davas, E. M., Tsirogianni, A., Kappou, I., Karamitsos, D., Economidou, I. and Dantis, P. C. (1999) Serum IL-6, TNF $\alpha$, p55 srTNF $\alpha$, p75 srTNF $\alpha$, srIL-2 $\alpha$ Levels 
and Disease Acitivity in Systemic Lupus Erythematosus. Clin. Rheumatol. 18, 1722.

149 Libby, P., Ridker, P. M. and Maseri, A. (2002) Inflammation and Atherosclerosis. Circulation 105, 1135-1143.

150 Boring, L., Gosling, J., Cleary, M. and Charo, I. F. (1998) Decreased lesion formation in CCR2-/- mice reveals a role for chemokines in the initiation of atherosclerosis. Nature 394, 894-897.

151 Tedgui, A. and Mallat, Z. (2006) Cytokines in Atherosclerosis: Pathogenic and Regulatory Pathways. Physiol. Rev. 86, 515-581.

152 Schlöndorff, D. and Banas, B. (2009) The mesangial cell revisited: no cell is an island. J. Am. Soc. Nephrol. JASN 20, 1179-1187.

153 Nee, L. E., McMorrow, T., Campbell, E., Slattery, C. and Ryan, M. P. (2004) TNFalpha and IL-1beta-mediated regulation of MMP-9 and TIMP-1 in renal proximal tubular cells. Kidney Int. 66, 1376-1386.

154 Yagi, H., Soto-Gutierrez, A., Navarro-Alvarez, N., Nahmias, Y., Goldwasser, Y., Kitagawa, Y., Tilles, A. W., Tompkins, R. G., Parekkadan, B. and Yarmush, M. L. (2010) Reactive Bone Marrow Stromal Cells Attenuate Systemic Inflammation via sTNFR1. Mol. Ther. 18, 1857-1864.

155 Choi, H. M., Jo, S.-K., Kim, S. H., Lee, J. W., Cho, E., Hyun, Y. Y., Cha, J. J., Kang, Y. S., Cha, D. R., Cho, W. Y., et al. (2013) Glucocorticoids attenuate septic acute kidney injury. Biochem. Biophys. Res. Commun. 435, 678-684. 
156 Pascual, V., Allantaz, F., Arce, E., Punaro, M. and Banchereau, J. (2005) Role of interleukin-1 (IL-1) in the pathogenesis of systemic onset juvenile idiopathic arthritis and clinical response to IL-1 blockade. J. Exp. Med. 201, 1479-1486.

157 Clinton, S. K., Underwood, R., Hayes, L., Sherman, M. L., Kufe, D. W. and Libby, P. (1992) Macrophage colony-stimulating factor gene expression in vascular cells and in experimental and human atherosclerosis. Am. J. Pathol. 140, 301-316.

158 Kleemann, R., Zadelaar, S. and Kooistra, T. (2008) Cytokines and atherosclerosis: a comprehensive review of studies in mice. Cardiovasc. Res. 79, 360-376.

159 Pan, X., Huang, L., Chen, J., Dai, Y. and Chen, X. (2011) Analysis of synovial fluid in knee joint of osteoarthritis:5 proteome patterns of joint inflammation based on matrix-assisted laser desorption/ionization time-of-flight mass spectrometry. Int. Orthop.

160 Dieudé, M., Senécal, J.-L. and Raymond, Y. (2004) Induction of endothelial cell apoptosis by heat-shock protein 60-reactive antibodies from anti-endothelial cell autoantibody-positive systemic lupus erythematosus patients. Arthritis Rheum. 50, $3221-3231$.

161 Schett, G., Xu, Q., Amberger, A., Van der Zee, R., Recheis, H., Willeit, J. and Wick, G. (1995) Autoantibodies against heat shock protein 60 mediate endothelial cytotoxicity. J. Clin. Invest. 96, 2569-2577.

162 Qazi, K. R., Torregrosa Paredes, P., Dahlberg, B., Grunewald, J., Eklund, A. and Gabrielsson, S. (2010) Proinflammatory exosomes in bronchoalveolar lavage fluid of patients with sarcoidosis. Thorax 65, 1016-1024. 
163 Fietta, A., Bardoni, A., Salvini, R., Passadore, I., Morosini, M., Cavagna, L., Codullo, V., Pozzi, E., Meloni, F. and Montecucco, C. (2006) Analysis of bronchoalveolar lavage fluid proteome from systemic sclerosis patients with or without functional, clinical and radiological signs of lung fibrosis. Arthritis Res. Ther. 8, R160.

164 Rottoli, P., Magi, B., Cianti, R., Bargagli, E., Vagaggini, C., Nikiforakis, N., Pallini, V. and Bini, L. (2005) Carbonylated proteins in bronchoalveolar lavage of patients with sarcoidosis, pulmonary fibrosis associated with systemic sclerosis and idiopathic pulmonary fibrosis. Proteomics 5, 2612-2618.

165 Bargagli, E., Penza, F., Vagaggini, C., Magi, B., Perari, M. G. and Rottoli, P. (2007) Analysis of carbonylated proteins in bronchoalveolar lavage of patients with diffuse lung diseases. Lung 185, 139-144.

166 Bargagli, E., Olivieri, C., Prasse, A., Bianchi, N., Magi, B., Cianti, R., Bini, L. and Rottoli, P. (2008) Calgranulin B (S100A9) levels in bronchoalveolar lavage fluid of patients with interstitial lung diseases. Inflammation 31, 351-354.

167 Tamburro, D., Facchiano, F., Petricoin, E. F., Liotta, L. A. and Zhou, W. (2010) Mass spectrometry-based characterization of the vitreous phosphoproteome. Proteomics Clin. Appl. 4, 839-846.

168 Schiess, R., Wollscheid, B. and Aebersold, R. (2009) Targeted proteomic strategy for clinical biomarker discovery. Mol. Oncol. 3, 33-44.

169 Liao, H., Wu, J., Kuhn, E., Chin, W., Chang, B., Jones, M. D., O’Neil, S., Clauser, K. R., Karl, J., Hasler, F., et al. (2004) Use of mass spectrometry to identify protein 
biomarkers of disease severity in the synovial fluid and serum of patients with rheumatoid arthritis. Arthritis Rheum. 50, 3792-3803.

170 Whiteaker, J. R., Zhao, L., Abbatiello, S. E., Burgess, M., Kuhn, E., Lin, C., Pope, M. E., Razavi, M., Anderson, N. L., Pearson, T. W., et al. (2011) Evaluation of large scale quantitative proteomic assay development using peptide affinity-based mass spectrometry. Mol. Cell. Proteomics MCP 10, M110.005645.

171 Bhinder, S., Singh, A. and Majithia, V. (2010) Membranous (class V) renal disease in systemic lupus erythematosus may be more common than previously reported: results of a 6-year retrospective analysis. Am. J. Med. Sci. 339, 230-232.

172 Brosius, F. C., Alpers, C. E., Bottinger, E. P., Breyer, M. D., Coffman, T. M., Gurley, S. B., Harris, R. C., Kakoki, M., Kretzler, M., Leiter, E. H., et al. (2009) Mouse Models of Diabetic Nephropathy. J. Am. Soc. Nephrol. 20, 2503-2512.

173 Petri, M., Orbai, A.-M., Alarcón, G. S., Gordon, C., Merrill, J. T., Fortin, P. R., Bruce, I. N., Isenberg, D., Wallace, D. J., Nived, O., et al. (2012) Derivation and validation of the Systemic Lupus International Collaborating Clinics classification criteria for systemic lupus erythematosus. Arthritis Rheum. 64, 2677-2686.

174 Wu, C. J., Conze, D. B., Li, T., Srinivasula, S. M. and Ashwell, J. D. (2006) Sensing of Lys 63-linked polyubiquitination by NEMO is a key event in NF-kappaB activation [corrected]. Nat Cell Biol 8, 398-406.

175 Ea, C. K., Deng, L., Xia, Z. P., Pineda, G. and Chen, Z. J. (2006) Activation of IKK by TNFalpha requires site-specific ubiquitination of RIP1 and polyubiquitin binding by NEMO. Mol Cell 22, 245-57. 
176 Ikeda, F., Deribe, Y. L., Skanland, S. S., Stieglitz, B., Grabbe, C., Franz-Wachtel, M., van Wijk, S. J., Goswami, P., Nagy, V., Terzic, J., et al. (2011) SHARPIN forms a linear ubiquitin ligase complex regulating NF-kappaB activity and apoptosis. Nature 471, 637-41.

177 Ikezumi, Y., Hurst, L., Atkins, R. C. and Nikolic-Paterson, D. J. (2004) Macrophage-mediated renal injury is dependent on signaling via the JNK pathway. J Am Soc Nephrol 15, 1775-84.

178 Sheryanna, A., Bhangal, G., McDaid, J., Smith, J., Manning, A., Foxwell, B. M., Feldmann, M., Cook, H. T., Pusey, C. D. and Tam, F. W. (2007) Inhibition of p38 mitogen-activated protein kinase is effective in the treatment of experimental crescentic glomerulonephritis and suppresses monocyte chemoattractant protein-1 but not IL-1beta or IL-6. J Am Soc Nephrol 18, 1167-79.

179 Stambe, C., Atkins, R. C., Tesch, G. H., Kapoun, A. M., Hill, P. A., Schreiner, G. F. and Nikolic-Paterson, D. J. (2003) Blockade of p38alpha MAPK ameliorates acute inflammatory renal injury in rat anti-GBM glomerulonephritis. J Am Soc Nephrol 14, 338-51.

180 Stambe, C., Nikolic-Paterson, D. J., Hill, P. A., Dowling, J. and Atkins, R. C. (2004) p38 Mitogen-activated protein kinase activation and cell localization in human glomerulonephritis: correlation with renal injury. J Am Soc Nephrol 15, 326-36.

181 Kawasaki, A., Ito, S., Furukawa, H., Hayashi, T., Goto, D., Matsumoto, I., Kusaoi, M., Ohashi, J., Graham, R. R., Matsuta, K., et al. (2010) Association of TNFAIP3 interacting protein 1, TNIP1 with systemic lupus erythematosus in a Japanese population: a case-control association study. Arthritis Res Ther 12, R174-R174. 
182 He, C. F., Liu, Y. S., Cheng, Y. L., Gao, J. P., Pan, T. M., Han, J. W., Quan, C., Sun, L. D., Zheng, H. F., Zuo, X. B., et al. (2010) TNIP1, SLC15A4, ETS1, RasGRP3 and IKZF1 are associated with clinical features of systemic lupus erythematosus in a Chinese Han population. Lupus 19, 1181-6.

183 Saran, R., Li, Y., Robinson, B., Ayanian, J., Balkrishnan, R., Bragg-Gresham, J., Chen, J. T. L., Cope, E., Gipson, D., He, K., et al. (2015) US Renal Data System 2014 Annual Data Report: Epidemiology of Kidney Disease in the United States. Am. J. Kidney Dis. Off. J. Natl. Kidney Found. 66, Svii, S1-305.

184 Caster, D. J., Korte, E. A., Nanda, S. K., McLeish, K. R., Oliver, R. K., G’sell, R. T., Sheehan, R. M., Freeman, D. W., Coventry, S. C., Kelly, J. A., et al. (2013) ABIN1 dysfunction as a genetic basis for lupus nephritis. J. Am. Soc. Nephrol. JASN 24, 1743-1754.

185 Fu, Y., Du, Y. and Mohan, C. (2007) Experimental anti-GBM disease as a tool for studying spontaneous lupus nephritis. Clin. Immunol. Orlando Fla 124, 109-118.

186 Klebanoff, S. J. (2005) Myeloperoxidase: friend and foe. J. Leukoc. Biol. 77, 598625.

187 Kim, Y. H., Goyal, M., Kurnit, D., Wharram, B., Wiggins, J., Holzman, L., Kershaw, D. and Wiggins, R. (2001) Podocyte depletion and glomerulosclerosis have a direct relationship in the PAN-treated rat. Kidney Int. 60, 957-968.

188 Uriarte, S. M., Rane, M. J., Luerman, G. C., Barati, M. T., Ward, R. A., Nauseef, W. M. and McLeish, K. R. (2011) Granule exocytosis contributes to priming and activation of the human neutrophil respiratory burst. J. Immunol. Baltim. Md 1950 187, 391-400. 
189 Bai, J., Tang, L., Lomas-Neira, J., Chen, Y., McLeish, K. R., Uriarte, S. M., Chung, C.-S. and Ayala, A. (2015) TAT-SNAP-23 treatment inhibits the priming of neutrophil functions contributing to shock and/or sepsis-induced extra-pulmonary acute lung injury. Innate Immun. 21, 42-54.

190 Uriarte, S. M., Rane, M. J., Merchant, M. L., Jin, S., Lentsch, A. B., Ward, R. A. and McLeish, K. R. (2013) Inhibition of neutrophil exocytosis ameliorates acute lung injury in rats. Shock Augusta Ga 39, 286-292.

191 Caster, D. J., Hobeika, L., Klein, J. B., Powell, D. W. and McLeish, K. R. (2015) Changing the concepts of immune-mediated glomerular diseases through proteomics. Proteomics Clin. Appl. 9, 967-971.

192 Bruschi, M., Sinico, R. A., Moroni, G., Pratesi, F., Migliorini, P., Galetti, M., Murtas, C., Tincani, A., Madaio, M., Radice, A., et al. (2014) Glomerular autoimmune multicomponents of human lupus nephritis in vivo: $\alpha$-enolase and annexin AI. J. Am. Soc. Nephrol. JASN 25, 2483-2498.

193 Bruschi, M., Galetti, M., Sinico, R. A., Moroni, G., Bonanni, A., Radice, A., Tincani, A., Pratesi, F., Migliorini, P., Murtas, C., et al. (2015) Glomerular Autoimmune Multicomponents of Human Lupus Nephritis In Vivo (2): Planted Antigens. J. Am. Soc. Nephrol. JASN 26, 1905-1924.

194 Caster, D. J., Korte, E. A., Merchant, M. L., Klein, J. B., Wilkey, D. W., Rovin, B. H., Birmingham, D. J., Harley, J. B., Cobb, B. L., Namjou, B., et al. (2015) Autoantibodies targeting glomerular annexin A2 identify patients with proliferative lupus nephritis. Proteomics Clin. Appl. 
195 Onishi, S., Adnan, E., Ishizaki, J., Miyazaki, T., Tanaka, Y., Matsumoto, T., Suemori, K., Shudou, M., Okura, T., Takeda, H., et al. (2015) Novel Autoantigens Associated with Lupus Nephritis. PloS One 10, e0126564.

196 Pusey, C. D. (2003) Anti-glomerular basement membrane disease. Kidney Int. 64, $1535-1550$.

197 Xie, C., Sharma, R., Wang, H., Zhou, X. J. and Mohan, C. (2004) Strain distribution pattern of susceptibility to immune-mediated nephritis. J. Immunol. Baltim. Md 1950 172, 5047-5055.

198 Xie, C., Zhou, X. J., Liu, X. and Mohan, C. (2003) Enhanced susceptibility to endorgan disease in the lupus-facilitating NZW mouse strain. Arthritis Rheum. 48, 1080-1092.

199 Rovin, B. H., Rumancik, M., Tan, L. and Dickerson, J. (1994) Glomerular expression of monocyte chemoattractant protein-1 in experimental and human glomerulonephritis. Lab. Investig. J. Tech. Methods Pathol. 71, 536-542.

200 Sinniah, R., Rui-Mei, L. and Kara, A. (1999) Up-regulation of cytokines in glomerulonephritis associated with murine malaria infection. Int. J. Exp. Pathol. 80, 87-95.

201 Gabay, C., Cakir, N., Moral, F., Roux-Lombard, P., Meyer, O., Dayer, J. M., Vischer, T., Yazici, H. and Guerne, P. A. (1997) Circulating levels of tumor necrosis factor soluble receptors in systemic lupus erythematosus are significantly higher than in other rheumatic diseases and correlate with disease activity. J. Rheumatol. 24, 303-308. 
202 Idasiak-Piechocka, I., Oko, A., Pawliczak, E., Kaczmarek, E. and Czekalski, S. (2010) Urinary excretion of soluble tumour necrosis factor receptor 1 as a marker of increased risk of progressive kidney function deterioration in patients with primary chronic glomerulonephritis. Nephrol. Dial. Transplant. Off. Publ. Eur. Dial. Transpl. Assoc. - Eur. Ren. Assoc. 25, 3948-3956.

203 Brennan, D. C., Yui, M. A., Wuthrich, R. P. and Kelley, V. E. (1989) Tumor necrosis factor and IL-1 in New Zealand Black/White mice. Enhanced gene expression and acceleration of renal injury. J. Immunol. Baltim. Md 1950 143, $3470-3475$.

204 Boswell, J. M., Yui, M. A., Burt, D. W. and Kelley, V. E. (1988) Increased tumor necrosis factor and IL-1 beta gene expression in the kidneys of mice with lupus nephritis. J. Immunol. Baltim. Md 1950 141, 3050-3054.

205 Neale, T. J., Rüger, B. M., Macaulay, H., Dunbar, P. R., Hasan, Q., Bourke, A., Murray-McIntosh, R. P. and Kitching, A. R. (1995) Tumor necrosis factor-alpha is expressed by glomerular visceral epithelial cells in human membranous nephropathy. Am. J. Pathol. 146, 1444-1454.

206 Abkhezr, M., Kim, E. Y., Roshanravan, H., Nikolos, F., Thomas, C., Hagmann, H., Benzing, T. and Dryer, S. E. (2015) Pleiotropic signaling evoked by tumor necrosis factor in podocytes. Am. J. Physiol. Renal Physiol. 309, F98-108.

207 Simpson, I. J., Amos, N., Evans, D. J., Thomson, N. M. and Peters, D. K. (1975) Guinea-pig nephrotoxic nephritis. I. The role of complement and polymorphonuclear leucocytes and the effect of antibody subclass and fragments in the heterologous phase. Clin. Exp. Immunol. 19, 499-511. 
208 Holdsworth, S. R. and Tipping, P. G. (2007) Leukocytes in glomerular injury. Semin. Immunopathol. 29, 355-374.

209 Lominadze, G., Powell, D. W., Luerman, G. C., Link, A. J., Ward, R. A. and McLeish, K. R. (2005) Proteomic analysis of human neutrophil granules. Mol. Cell. Proteomics MCP 4, 1503-1521.

210 Uriarte, S. M., Powell, D. W., Luerman, G. C., Merchant, M. L., Cummins, T. D., Jog, N. R., Ward, R. A. and McLeish, K. R. (2008) Comparison of proteins expressed on secretory vesicle membranes and plasma membranes of human neutrophils. J. Immunol. Baltim. Md 1950 180, 5575-5581.

211 Sistani, L., Dunér, F., Udumala, S., Hultenby, K., Uhlen, M., Betsholtz, C., Tryggvason, K., Wernerson, A. and Patrakka, J. (2011) Pdlim2 is a novel actinregulating protein of podocyte foot processes. Kidney Int. 80, 1045-1054.

212 Akilesh, S., Suleiman, H., Yu, H., Stander, M. C., Lavin, P., Gbadegesin, R., Antignac, C., Pollak, M., Kopp, J. B., Winn, M. P., et al. (2011) Arhgap24 inactivates Rac1 in mouse podocytes, and a mutant form is associated with familial focal segmental glomerulosclerosis. J. Clin. Invest. 121, 4127-4137.

213 Faul, C., Donnelly, M., Merscher-Gomez, S., Chang, Y. H., Franz, S., Delfgaauw, J., Chang, J.-M., Choi, H. Y., Campbell, K. N., Kim, K., et al. (2008) The actin cytoskeleton of kidney podocytes is a direct target of the antiproteinuric effect of cyclosporine A. Nat. Med. 14, 931-938.

214 Santamaria, B., Marquez, E., Lay, A., Carew, R. M., González-Rodríguez, Á., Welsh, G. I., Ni, L., Hale, L. J., Ortiz, A., Saleem, M. A., et al. (2015) IRS2 and 
PTEN are key molecules in controlling insulin sensitivity in podocytes. Biochim. Biophys. Acta 1853, 3224-3234.

215 Yuan, H., Takeuchi, E. and Salant, D. J. (2002) Podocyte slit-diaphragm protein nephrin is linked to the actin cytoskeleton. Am. J. Physiol. Renal Physiol. 282, F585-591.

216 Bollée, G., Flamant, M., Schordan, S., Fligny, C., Rumpel, E., Milon, M., Schordan, E., Sabaa, N., Vandermeersch, S., Galaup, A., et al. (2011) Epidermal growth factor receptor promotes glomerular injury and renal failure in rapidly progressive crescentic glomerulonephritis. Nat. Med. 17, 1242-1250.

217 Endlich, N., Kress, K. R., Reiser, J., Uttenweiler, D., Kriz, W., Mundel, P. and Endlich, K. (2001) Podocytes respond to mechanical stress in vitro. J. Am. Soc. Nephrol. JASN 12, 413-422.

218 Morigi, M., Buelli, S., Angioletti, S., Zanchi, C., Longaretti, L., Zoja, C., Galbusera, M., Gastoldi, S., Mundel, P., Remuzzi, G., et al. (2005) In response to protein load podocytes reorganize cytoskeleton and modulate endothelin-1 gene: implication for permselective dysfunction of chronic nephropathies. Am. J. Pathol. 166, 13091320.

219 Morigi, M., Buelli, S., Zanchi, C., Longaretti, L., Macconi, D., Benigni, A., Moioli, D., Remuzzi, G. and Zoja, C. (2006) Shigatoxin-induced endothelin-1 expression in cultured podocytes autocrinally mediates actin remodeling. Am. J. Pathol. 169, 1965-1975. 
220 Danilewicz, M. and Wągrowska-Danilewicz, M. (2013) The immunoexpression of glomerular NF- $\mathrm{B}$ in proteinuric patients with proliferative and non-proliferative glomerulopathies. Pol. J. Pathol. Off. J. Pol. Soc. Pathol. 64, 78-83.

221 G'Sell, R. T., Gaffney, P. M. and Powell, D. W. (2015) A20-Binding Inhibitor of

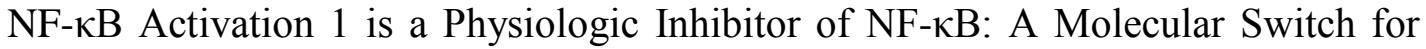
Inflammation and Autoimmunity. Arthritis Rheumatol. Hoboken NJ 67, 2292-2302.

222 Bomback, A. S. and Appel, G. B. (2010) Updates on the Treatment of Lupus Nephritis. J. Am. Soc. Nephrol. 21, 2028-2035.

223 Bomback, A. S. and Gharavi, A. G. (2013) Lupus nephritis: Ancestry, genetic risk and health disparities. Nat. Rev. Nephrol. 9, 699-700. 


\title{
CURRICULUM VITAE
}

\author{
Erik A. Korte \\ 821 Packard Ave.
}

Louisville, KY 40217

Phone: (417) 496-3697

Fax: (502) 852-4384

eakorte@gmail.com

\section{EDUCATION AND TRAINING:}

07/2012 - current Ph.D. Candidate - Biochemistry and Molecular Biology University of Louisville, Louisville, KY

Advisor: David W. Powell, Ph.D.

Dissertation Title: "ABIN1 in the pathogenesis of glomerulonephritis and the novel podocyte-neutrophil proinflammatory axis"

*Dissertation Defense scheduled 04/08/16

*Graduation expected May 2016 


\begin{abstract}
08/2010-07/2012 M.S. - Biochemistry and Molecular Biology
University of Louisville, Louisville, KY

Advisor: David W. Powell, Ph.D.

08/1999-12/2003 B.S. - Biology

Missouri State University, Springfield, MO
\end{abstract}

\title{
EMPLOYMENT:
}

2009-2010

2006-2008

2002-2006

2001-2006
Director, Research and Development, Boyce and Bynum Pathology Laboratories, Columbia, MO

Kaplan Test Prep, Columbia, MO

Gross Human Anatomy Instructor and Lab Coordinator, Missouri

State University

St. John's Regional Health Center Emergency Trauma Center Technician, Springfield, MO

\section{TEACHING ACTIVITIES:}

2011

2006-2008
Teaching Assistant, Advanced Biochemical Methods, Department of Biochemistry and Molecular Biology, University of Louisville Kaplan Test Prep, Columbia, MO 


\section{AWARDS:}

$2^{\text {nd }}$ Place Overall, University of Louisville, Research! Louisville, 2015.

American Society of Nephrology, Kidney STARS Program and Travel Award, 2014.

American Heart Association, Predoctoral Fellowship, 2014-2016.

Finalist, Poster Competition, University of Louisville, Research! Louisville, 2014.

University of Louisville, Research Committee Travel Award, March 2014.

$1^{\text {st }}$ Place, Graduate Research Competition, Cellular and Molecular Biology, Kentucky Academy of Sciences Annual Meeting, Morehead State University, November 2013.

Finalist, Poster Competition, University of Louisville, Research! Louisville, 2013.

$1^{\text {st }}$ Place, Overall Poster Competition, University of Louisville, Biochemistry and Molecular Biology Research Colloquium, 2013.

Michael Gordon Fellowship Awardee, 2013.

Formally Commissioned as a Kentucky Colonel by Governor Steven Beshear, March 13, 2012.

\section{MENTORING:}


Ryan Sheehan, M.S. is a current Ph.D. student in Biochemistry and Molecular Biology that completed a rotation in the Powell lab Fall Semester 2012. Ryan has now joined the lab and is working toward developing an independent project. I have trained him in lab techniques for cell culture, protein purification, western blotting, RT-qPCR, and other techniques.

Rachel G’Sell, M.S. was a Masters student in Biochemistry and Molecular Biology that completed a rotation in the Powell lab Fall Semester 2012. She joined the lab and completed a masters project before moving to the University of Missouri to begin a physical therapy program. I have trained her in lab techniques for cell culture, protein purification, western blotting, RT-qPCR, ELISA, immunofluorescence, and other techniques.

Rebecca Oliver, B.S. was a summer research undergraduate student in the Powell Lab in 2012.

Dawn Caster, M.D. is a Nephrology Attending in the Kidney Disease Program at the University of Louisville School of Medicine that has completed a one-year research fellowship (7/2012-7/2013) in the Powell lab and is now starting a clinical/translational research program in glomerular disease. 
Katie Harris was a middle school student at St. Francis of Assisi who completed an $8^{\text {th }}$ grade science project under my guidance which won local, regional and Kentucky State science fairs.

Rose Chancy is a middle school student at St. Francis of Assisi who completed her $7^{\text {th }}$, $8^{\text {th }}$ and $9^{\text {th }}$ grade science projects under my guidance which won local and regional science fairs and placed second at the Kentucky State science fair in 2012 and first place in 2013 followed by a top 30 finish nationally.

\section{COMMUNITY SERVICE:}

2012-2014

Judge - Manual Regional Science Fair (grades 9-12)

2012-2013

Judge - St. Francis of Assisi Science Fair

2012

Judge - Kentucky Orchid Society Annual Show

\section{PROFESSIONAL MEMBERSHIPS:}

American Heart Association

American Society of Nephrology

Kentucky Academy of Sciences

American Association for the Advancement of Science

American Society for Biochemistry and Molecular Biology

Society for Experimental Biology and Medicine 


\section{ADMINISTRATIVE SERVICE:}

Biochemistry and Molecular Biology Student Body President

Biochemistry and Molecular Biology Student Body Vice President

University of Louisville School of Medicine Strategic Planning Steering Committee

Graduate Executive Committee

Council of the School of Medicine

Department of Microbiology and Immunology Chair Search Committee

Interviews with Department of Biochemistry and Molecular Biology Graduate School

Candidates

Organization of the Recruitment Weekend for Prospective Biochemistry Students

\section{EXTRACURRICULAR:}

2013-2016

2011-2014

2010-2016

2010-2012

2002-2003

2001, 2003

1999-2003

1999-2003
Derby City Mustang Club

Kentucky Orchid Society

Louisville Metro Government Kickball League

Louisville Masters Swim Team

Varsity Swimming Captain

All-Conference, Men’s Swimming

Varsity Swimming and Diving Team

Academic All-Conference 


\section{GRANT SUPPORT:}

Granting Agency: American Heart Association (Predoctoral Fellowship, 14PRE19880002)

Period of Performance: 07/01/14-06/30/16

Title: ABIN1 function in the protection from lupus nephritis and cardiovascular disease

PI: Erik Korte $\quad 24$ Cal. Months (90\% effort) \$52,000

The overall objective of this project is to define the role of ABIN1 in renal cells in the promotion and pathogenesis of lupus nephritis.

\section{PRESENTATIONS:}

\section{$\underline{\text { Seminars }}$}

“ABIN1, Podocytes and the Glomerular Inflammatory Microenvironment”. University of Louisville, Department of Biochemistry and Molecular Biology Seminar Series. (September $\left.11^{\text {th }}, 2015\right)$ 
“ABIN1, Podocytes and the Glomerular Inflammatory Microenvironment”. University of Louisville, Kidney Disease Program Research Seminar Series. (September $9^{\text {th }}, 2015$ )

“ABINI and Lupus Nephritis: Why the Kidney Matters”. University of Louisville, Department of Biochemistry and Molecular Biology Seminar Series. (May 12 $2^{\text {th }}, 2014$ )

“ABIN1 and Lupus Nephritis: Why the Kidney Matters”. University of Louisville, Kidney Disease Program Research Seminar Series. (April $18^{\text {th }}, 2014$ )

“ABIN1 Dysfunction as a Basis for Lupus Nephritis”. Kentucky Academy of Sciences, Morehead State University, 2013. (November $\left.9^{\text {th }}, 2013\right) . \quad 1^{\text {st }}$ Place, Cellular and Molecular Biology.

“ABIN1 Dysfunction as a Basis for Lupus Nephritis”. University of Louisville, Biochemistry and Molecular Biology Research Colloquium, 2013. (August 23 ${ }^{\text {rd }}, 2013$ )

"Ubiquitin Binding by ABIN-1 Controls Inflammation and Development of Lupus Nephritis”. University of Louisville, Kidney Disease Program Research Seminar Series. (April 18 ${ }^{\text {th }}, 2013$ )

“Ubiquitin Binding by ABIN-1 Controls Multiple Signaling Pathways”. University of Louisville, Department of Biochemistry and Molecular Biology Seminar Series. (April $\left.8^{\text {th }}, 2013\right)$ 
"B7 Costimulation Blockade in Induced Allograft Tolerance”. University of Louisville, Biochemistry and Molecular Biology Journal Club (January $9^{\text {th }}$, 2012)

\section{$\underline{\text { Posters }}$}

\section{National/International Conferences}

Powell DW, Sheehan RM, Caster DJ, McLeish KR, Korte EA. A novel mechanism for enhanced neutrophil recruitment, retention, and podocyte damage in immune complexmediated glomerular disease. American Association of Immunology Meeting, Seattle, Washington , May 2016

Korte, EA; Sheehan, RM; Powell, DW. Loss of glomerular cell ABIN1 polyubiquitin binding results in enhanced pro-inflammatory signaling and kidney injury. Abstract and Poster Preparation. Mechanisms of Pro-Inflammatory Diseases, Keystone Symposia. Olympic Valley, CA, April 2015.

Powell, DW; G'Sell, RT; Sheehan, RM; Caster, DJ; Merchant, ML. McLeish, KR; Korte, EA. Disrupted ABIN1 Function Results in a Pro-Inflammatory Phenotype in 
Human Glomerular Cells. Abstract and Poster Presentation, American Society of Nephrology Annual Meeting, Philadelphia, PA. November 2014.

Korte, EA, G'Sell, RT; Sheehan, RM; Caster, DJ; Powell, DP. Dysfunction of polyubiquitin binding by ABIN1 as a basis for lupus nephritis. Abstract and Poster Presentation, Experimental Biology International Conference, San Diego, CA, April 2014.

Caster DJ, Korte, EA, Birmingham DJ, Joglekar AS, Klein JB, Harley JB, Namjou B, McLeish KR, Merchant ML, Rovin, BH, and Powell, DW. Patterns of Circulating Autoantibodies in Patients with Lupus Nephritis. Abstract and Poster Presentation, American Society of Nephrology, Kidney Week, Atlanta, GA, November 2013.

Korte, E.A., Cummins, T.D., Mendenhall, M.D., Barati, M.T., Klein, J.B., and Powell, D.W. Elongin C is a Mediator of Notch4 Activity in Human Renal Tubule Cells. Keystone Symposia, Whistler, Canada, March 2012.

Powell, D.W. Korte, E.A., Cohen, P., and Nanda, S.K. Disruption of ABIN1 inhibition of NF-kB activity causes lupus-associated glomerulonephritis. Keystone Symposia, Whistler, Canada, March 2012.

Caster DJ, Birmingham DJ, Klein JB, Harley JB, Korte EA, McLeish KR, Merchant ML, Rovin, BH, and Powell, DW. Identification of Candidate Target Antigens for 
Membranous Lupus Nephritis. American Society of Nephrology Kidney Week, San Diego, CA, November 2012.

\section{Local/Regional Conferences}

Korte, Erik; Sheehan, Ryan; Caster, Dawn; Powell, David. Loss of glomerular ABIN1 polyubiquitin binding results in enhanced pro-inflammatory signaling and kidney injury. University of Louisville, Research! Louisville, 2014. $2^{\text {nd }}$ Place Overall, Poster Competition.

Korte, Erik; Sheehan, Ryan; Powell, David. Loss of glomerular cell ABIN1 polyubiquitin binding results in enhanced pro-inflammatory signaling and kidney injury. University of Louisville, Biochemistry and Molecular Genetics Research Colloquium, 2015.

Korte, Erik; Sheehan, Ryan; Caster, Dawn; G’Sell, Rachel; Powell, David. Loss of ABIN1 Function Results in a Pro-Inflammatory Phenotype in Human Glomerular Cells. University of Louisville, Research! Louisville, 2014. Finalist, Poster Competition.

Korte, Erik; Sheehan, Ryan; Caster, Dawn; G’Sell, Rachel; Powell, David. ABIN1 Dysfunction as a Basis for Lupus Nephritis. University of Louisville, Research! Louisville, 2013. Finalist, Poster Competition. 
Joglekar AS, Korte, EA, Caster DJ, Klein JB, Rovin BH, Harley JB, Merchant ML, McLeish KR, Stribinskis V, G’Sell RT, Sheehan RM, and Powell, DW. Target Antigens in Membranous Lupus Nephritis. Abstract and Poster Presentation, Research! Louisville, Louisville, KY, September 2013.

Korte, Erik; Sheehan, Ryan; Caster, Dawn; G’Sell, Rachel; Powell, David. ABIN1 Dysfunction as a Basis for Lupus Nephritis. University of Louisville, Biochemistry and Molecular Biology Research Colloquium, 2013. $1^{\text {st }}$ Place Poster Graduate Student, $1^{\text {st }}$ Place Poster Overall.

Korte, E.A., Cummins, T.D., Mendenhall, M.D., Barati, M.T., Klein, J.B., and Powell, D.W. Elongin C is a Mediator of Notch4 Activity in Human Renal Tubule Cells. University of Louisville, Biochemistry and Molecular Biology Retreat, 2011.

Caster DJ, Klein JB, Harley JB, Korte EA, McLeish KR, Merchant ML, Oliver R, Rovin $\mathrm{BH}$, and Powell DW. Identification of GFR $\alpha 1$ as a Candidate Target Antigen in Membranous Lupus Nephritis. Research Louisville!, 2012, $1^{\text {st }}$ place poster, clinical fellow

\section{PUBLICATIONS:}

Caster DJ, Korte EA, Merchant ML, Klein JB, Wilkey DW, Rovin BH, Birmingham DJ, Harley JB, Cobb BL, Namjou B, McLeish KR, Powell DW. “Autoantibodies targeting 
glomerular annexin A2 identify patients with proliferative lupus nephritis.” Proteomics Clinical Applications. 2015 Dec;9(11-12):1012-20. PMID: 25824007.

D. J. Caster* and E. A. Korte*, S. K. Nanda, K. R. McLeish, R. K. Oliver, R. T. G'sell, R. M. Sheehan, D. W. Freeman, S. C. Coventry, J. A. Kelly, J. M. Guthridge, J. A. James, K. L. Sivils, M. E. Alarcon-Riquelme, R. H. Scofield, I. Adrianto, P. M. Gaffney, A. M. Stevens, B. I. Freedman, C. D. Langefeld, B. P. Tsao, B. A. Pons-Estel, C. O. Jacob, D. L. Kamen, G. S. Gilkeson, E. E. Brown, G. S. Alarcon, J. C. Edberg, R. P. Kimberly, J. Martin, J. T. Merrill, J. B. Harley, K. M. Kaufman, J. D. Reveille, J.-M. Anaya, L. A. Criswell, L. M. Vila, M. Petri, R. Ramsey-Goldman, S.-C. Bae, S. A. Boackle, T. J. Vyse, T. B. Niewold, P. Cohen, and D. W. Powell, “ABIN1 Dysfunction as a Genetic Basis for Lupus Nephritis,” J. Am. Soc. Nephrol. JASN, 2013 Nov;24(11):1743-54. PMID: 23970121.

*Equal Contributing First Authors

Korte EA, Gaffney PM, Powell DW. (2011) Proteomic applications for defining cellular mechanisms and diagnostic markers of Systemic Lupus Erythematosus. Arthritis Research \& Therapy. 2012 Feb 20;14(1):204. PMID: 22364570.

Cummins TD, Mendenhall MD, Lowry MN, Korte EA, Barati MT, Khundmiri SJ, Salyer SA, Klein JB, Powell DW. (2011) Elongin C Is A Mediator Of Notch4 Activity In Human Renal Tubule Cells. Biochim Biophys Acta. 1814,1748-1757. 